BNL-114389-2017-BC

\title{
Risk Importance Measures in the Design and Operation of Nuclear Power Plants
}

\author{
I. Vrbanic, P. Samanta
}

To be published in "Nuclear Engineering and Technology for the $21^{\text {st }}$ Century"

October 2017

Nuclear Science and Technology Department

Brookhaven National Laboratory

\section{U.S. Department of Energy American Society of Mechanical Engineers (ASME)}

Notice: This manuscript has been authored by employees of Brookhaven Science Associates, LLC under Contract No. DE-SC0012704 with the U.S. Department of Energy. The publisher by accepting the manuscript for publication acknowledges that the United States Government retains a non-exclusive, paid-up, irrevocable, world-wide license to publish or reproduce the published form of this manuscript, or allow others to do so, for United States Government purposes. 


\section{DISCLAIMER}

This report was prepared as an account of work sponsored by an agency of the United States Government. Neither the United States Government nor any agency thereof, nor any of their employees, nor any of their contractors, subcontractors, or their employees, makes any warranty, express or implied, or assumes any legal liability or responsibility for the accuracy, completeness, or any third party's use or the results of such use of any information, apparatus, product, or process disclosed, or represents that its use would not infringe privately owned rights. Reference herein to any specific commercial product, process, or service by trade name, trademark, manufacturer, or otherwise, does not necessarily constitute or imply its endorsement, recommendation, or favoring by the United States Government or any agency thereof or its contractors or subcontractors. The views and opinions of authors expressed herein do not necessarily state or reflect those of the United States Government or any agency thereof. 
Nuclear Engineering and Technology for the 21st Century - Monograph Series

\section{Risk Importance}

Measures in the Design and Operation of

Nuclear Power Plants

Ivan Vrbanic, Pranab Samanta,

Ivica Basic 
(C) 2017, The American Society of Mechanical Engineers (ASME), 2 Park Avenue, New York, NY 10016, USA (www.asme.org)

All rights reserved. Printed in the United States of America. Except as permitted under the United States Copyright Act of 1976, no part of this publication may be reproduced or distributed in any form or by any means, or stored in a database or retrieval system, without the prior written permission of the publisher.

INFORMATION CONTAINED IN THIS WORK HAS BEEN OBTAINED BY THE AMERICAN SOCIETY OF MECHANICAL ENGINEERS FROM SOURCES BELIEVED TO BE RELIABLE. HOWEVER, NEITHER ASME NOR ITS AUTHORS OR EDITORS GUARANTEE THE ACCURACY OR COMPLETENESS OF ANY INFORMATION PUBLISHED IN THIS WORK. NEITHER ASME NOR ITS AUTHORS AND EDITORS SHALL BE RESPONSIBLE FOR ANY ERRORS, OMISSIONS, OR DAMAGES ARISING OUT OF THE USE OF THIS INFORMATION. THE WORK IS PUBLISHED WITH THE UNDERSTANDING THAT ASME AND ITS AUTHORS AND EDITORS ARE SUPPLYING INFORMATION BUT ARE NOT ATTEMPTING TO RENDER ENGINEERING OR OTHER PROFESSIONAL SERVICES. IF SUCH ENGINEERING OR PROFESSIONAL SERVICES ARE REQUIRED, THE ASSISTANCE OF AN APPROPRIATE PROFESSIONAL SHOULD BE SOUGHT.

ASME shall not be responsible for statements or opinions advanced in papers or ... printed in its publications (B7.1.3). Statement from the Bylaws.

For authorization to photocopy material for internal or personal use under those circumstances not falling within the fair use provisions of the Copyright Act, contact the Copyright Clearance Center (CCC), 222 Rosewood Drive, Danvers, MA 01923, tel: 978-750-8400, www.copyright.com.

Requests for special permission or bulk reproduction should be addressed to the ASME Publishing Department, or submitted online at https://www.asme.org/shop/books/book-proposals/permissions

ASME Press books are available at special quantity discounts to use as premiums or for use in corporate training programs. For more information, contact Special Sales at CustomerCare@asme.org

Library of Congress Cataloging-in-Publication Data

Names: Vrbanic, Ivan, author.| Samanta, Pranab, author. | Basic, Ivica, author.

Title: Risk importance measures in the design and operation of nuclear power plants / Ivan Vrbanic,

Pranab Samanta, Ivica Basic.

Description: New York : ASME Press, [2017] | Series: Nuclear engineering and technology for 21st century-monograph series | Includes bibliographical references and index.

Identifiers: LCCN 2017027159 | ISBN 9780791861394 (alk. paper)

Subjects: LCSH: Nuclear power plants--Design and construction. | Nuclear power plants--Risk assessment.| Nuclear power plants--Management.

Classification: LCC TK9152.16 .V73 2017 | DDC 621.48/32--dc23 LC record available at https://lccn .loc.gov/2017027159 


\section{Acknowledgement}

It is a pleasant duty to give our thanks and appreciation to the reviewers who not only undertook to read the manuscript but provided us with advices and suggestions which made the final "product" considerably better. 


\section{Series Editors' Preface}

\section{Nuclear Engineering and Technology for the 21st Century- Monographs Series}

Nuclear engineering and technology play a vital role in achieving low carbon emission goals worldwide, while providing reliable, baseload power to the world economy. Presently over 12 percent of the world's energy needs are satisfied by nuclear power-with 30 countries operating 436 nuclear power plants and 3 countries (France, Slovakia, and Belgium) using nuclear power to provide over half their power needs (source: Nuclear Energy Institute: http://www.nei,org).

The country with the largest number of operational nuclear power plants (the United States) has 102 plants and uses nuclear power to provide over 19 percent of its needs. Concurrently, the advanced nuclear power plant designs are the basis for extensive, ongoing research and development efforts in many countries with the promise of enhanced sustainability, safety, and proliferation-free power-sources with everhigher operational efficiencies and capacity factors. Consequently, there are many fruitful topics of interest in the nuclear engineering field to be addressed in this exciting monograph series.

The Nuclear Engineering and Technology for the 21st Century monograph series provides current and future engineers, researchers, technicians and other professionals and practitioners with practical, concise but key information concerning the nuclear technologies from areas of medical applications, mining, processing and manufacturing, environmental monitoring to safe and energy-efficient plant operation and electricity generation. Each monograph should provide a well rounded and definitive state-of-the-art review of its subject, with a focus on applied research and development, and best industry practices, processes and related technological applications. The series is envisaged as a collection of 80 to 100 pages monograph publications which can stand as the most authoritative source of information on current state of a topic, application or discipline. Core topics include, but are not limited to:

+ best practices in power plant operation

+ nuclear science and technology in medicine,

+ irradiation technologies and applications, 
- fuel cycle processes, engineering and technologies,

- nuclear reactor thermal hydraulics and/or neutronics

- materials for current and advance power generation

+ nuclear safety and environmental impact

+ next generation of nuclear power plants

+ radiation in our environment

+ radioecology, radiobiology, radiation chemistry

Series Editors:

Dr. Jovica Riznic, Canadian Nuclear Safety Commission

Dr. Richard Schultz, Idaho National Laboratory 


\section{Table of Contents}

Acknowledgement iii

Series Editor's Preface v v

Abstract ix

1. Introduction $\quad 1$

2. Safety analysis of NPP, the role of risk assessment, and measuring risk importance 3

3. Deterministic principles of safety importance and need for consideration of risk

4. Risk importance measures in NPP risk assessment 11

4.1 Background on importance measures in risk assessment 11

4.2 Importance measures broadly used in PRAs for NPPs 12

4.3 Examples of use of risk importance measures in risk-informed applications

4.3.1 Risk-informed categorization and treatment of SSCs for nuclear power reactors

4.3.2 Risk-informed changes to NPP technical specifications

4.3.3 Application in maintenance rule

4.3.4 Applications associated with ROP

4.4 Risk importance measures: focus of discussion in this monograph 25

5. Risk importance of component represented by a single basic event not involved in a CCF Group

5.1 Introductory considerations

5.2 Theoretical relation between RAW and RRW 34

$\begin{array}{ll}5.3 \text { FC for a single basic event } & 35\end{array}$

5.4 RAW for a single basic event $\quad 37$

5.5 RRW for a single basic event 39

5.6 Reliability importance for a single basic event 39

6. Risk importance of a component represented by multiple basic events, none involved in a CCF Group (FT module) 43

6.1 Introduction 43

6.2 FC, RAW, RRW and reliability importance for component-level FT module

6.3 Example: importance measures for a FT module for failure of a standby pump $\quad 45$

$\begin{array}{lll}6.3 .1 \text { FC } & 46\end{array}$

6.3.2 RAW, RRW and reliability importance 47

7. Risk importance of component represented by multiple different

FT modules or basic events in different accident scenarios, none involved in a CCF Group

7.1 Introduction 49

$\begin{array}{ll}7.2 \mathrm{FC} & 51\end{array}$ 
viii Risk Importance Measures in the Design and Operation of NPPs

7.3 RAW 52

7.4 RRW and reliability importance 54

7.5 Example: standby pump with two different operating
failure rates

7.5.1 FC $\quad 56$

7.5.2 RAW 56

7.5.3 RRW and reliability importance 57

8. Risk importance of component represented by a single basic event
(failure mode) involved in a CCF Group

8.1 Introduction $\quad 59$

8.2 Failure with CCF potential 60

8.2.1 FC 62

8.2.2 RAW 63

8.2.3 RRW and reliability importance 64

8.3 Unavailability with no impact on CCF 65

8.4 Particular CCF event 67

9. Risk importance of component represented by multiple basic events (failure modes) involved in CCF Groups $\quad 69$

9.1 Introduction 69

9.2 Failure with CCF potential 73

$\begin{array}{ll}\text { 9.2.1 RAW } & 73\end{array}$

$\begin{array}{ll}\text { 9.2.2 RRW } & 75\end{array}$

9.2.3 FC $\quad 75$

9.2.4 Reliability importance $\quad 76$

9.3 Unavailability with no impact on CCF 77

9.4 Particular CCF event 77

10. Demonstrative examples of the component-level importance
measure

11. Limitations of risk importance measures 87

12. Summary 93

13. References 97

$\begin{array}{ll}\text { Appendix A: } & \begin{array}{l}\text { Example from practice-calculation of risk importance } \\ \text { measures by a PRA computer code }\end{array} \\ & 101\end{array}$

Appendix B: Demonstrating examples based on a simplified PRA model 105

B.1 Description of simplified PRA model used for demonstration $\quad 105$

B.2 Examples concerning theoretical relation between RAW and RRW and importance measures for single basic event (Examples B1 through B4) 107

B.3 Examples of calculation of component-level importance measures 114 $\begin{array}{ll}\text { About the authors } & 137\end{array}$ 


\section{Abstract}

This monograph presents and discusses risk importance measures as quantified by the probabilistic risk assessment (PRA) models of nuclear power plants (NPPs) developed according to the current standards and practices. Usually, PRA tools calculate risk importance measures related to a single "basic event" representing particular failure mode. This is, then, reflected in many current PRA applications. The monograph focuses on the concept of "component-level" importance measures that take into account different failure modes of the component including common-cause failures (CCFs). In opening sections the role of risk assessment in safety analysis of an NPP is introduced and discussion given of "traditional", mainly deterministic, design principles which have been established to assign a level of importance to a particular system, structure or component. This is followed by an overview of main risk importance measures for risk increase and risk decrease from current PRAs. Basic relations which exist among the measures are shown. Some of the current practical applications of risk importance measures from the field of NPP design, operation and regulation are discussed. The core of the monograph provides a discussion on theoretical background and practical aspects of main risk importance measures at the level of "component" as modeled in a PRA, starting from the simplest case, single basic event, and going toward more complex cases with multiple basic events and involvements in CCF groups. The intent is to express the component-level importance measures via the importance measures and probabilities of the underlying single basic events, which are the inputs readily available from a PRA model and its results. Formulas are derived and discussed for some typical cases. The formulas and their results are demonstrated through some practical examples, done by means of a simplified PRA model developed in and run by RiskSpectrum ${ }^{\circledast}$ tool, which are presented in the appendices. The monograph concludes with discussion of limitations of the use of risk importance measures and a summary of component-level importance cases evaluated. 


\section{Introduction}

In using risk-informed approaches for ensuring safety of operating nuclear power plants (NPPs), risk importance measures obtained from probabilistic risk assessments (PRAs) of the plants are integral elements of consideration in many cases. Obtaining these measures in appropriate forms is helpful for decision makers and can facilitate the use of risk information.

There are many sources, from theoretical books to engineer's handbooks, which discuss the risk importance measures. In this monograph the focus is on risk importance as quantified by the PRA models of NPPs developed in accordance with current standards and tools. The concept of risk importance measure in PRA is, in many applications, related to a single "basic event" and this is what is usually calculated by the PRA tools (although some of them, such as RiskSpectrum ${ }^{\circledR}$, include certain advanced options, as discussed later). On the other hand, what is of interest in practical applications is risk importance of particular component such as pump or valve, which is in current PRA models typically represented by multiple basic events where each basic event is related to specific failure mode or cause of unavailability. The same failure mode may, due to different boundary conditions, be presented by different basic events in different accident sequences. To complicate the things further, failure modes may be shared by other components, i.e. representative basic event may be a member of some common cause failure (CCF) group. In order to map the importance of particular basic events into the importance of component, some PRA applications, discussed below, established a set of rules to be used for the purpose. This process is somewhat complicated, is subject to interpretation, and in some cases, requires additional evaluations. Availability of measures that can be directly connected to a component of a safety system, "componentlevel" importance measures, can simplify the use of these measures in many applications.

This monograph discusses the concept of "component-level" importance measures that take into account different failure modes of the component including common-cause failures and derives mathematical formulas for their quantification. Discussed measures can be calculated using the results from the current computerized PRA tools and 


\section{Risk Importance Measures in the Design and Operation of NPPs}

can be obtained and presented as part of the risk importance measures included in these PRA tools.

The monograph first makes an overview of main risk importance measures from current PRAs and shows some basic relations which exist among them. This is, then, followed by exploring the "componentlevel" risk importance and the ways it can be expressed via the importance measures of corresponding basic events which are reported by PRA tools, with due attention devoted to CCFs. Some basic relations and formulas derived through calculations are, also, demonstrated on the basis of a simplified PRA model.

Section 2 introduces, on a general level, the role of risk assessment in safety analysis of an NPP. Section 3 discusses traditional, mainly deterministic, principles which have been established to assign a level of importance to a particular system, structure or component (SSC). Section 4 introduces risk importance measures and describes some of their practical applications, mostly from the field of NPP operation and regulation. Sections 5 through 9 discuss theoretical background and practical aspects of main risk importance measures at the level of "component" as modeled in a PRA, starting from the simplest case (single basic event) and going toward more complex cases (multiple basic events and involvement in CCF groups). Section 10 summarizes the results of demonstrative examples while Section 11 discusses some specific aspects of the use of risk importance measures, including the limitations. Finally, the appendices are devoted to demonstrations and practical examples. They were done by means of a simplified PRA model, described in Section B.1 of Appendix B, developed in and run by RiskSpectrum ${ }^{\circledR}$ tool. 


\section{Safety analysis of NPP, the role of risk assessment, and measuring risk importance}

In order to be able to "measure" the importance of a particular feature or component which has certain safety function in any technical system (NPP included), an appropriate technical definition needs first to be established for a quantitative measure of the system's "safety". One of the, arguably, best ways is to present it through its inversion, the "risk", which is, in engineer's terms, quantitatively defined by the famous "risk curve" representing the probability (or frequency) of exceedance as a function of magnitude of consequences, Figure 2-1. This presentation corresponds, for example, to the complementary cumulative distribution function used to depict the risk in NUREG-1150 [1].

The overall risk is defined by the area below the risk curve, i.e.:

$$
R=\int_{0}^{\infty}\left|C\left(P_{E}\right) d P_{E}\right| \cdot
$$

The purpose of the "safety management" or "risk management" in NPP design and operation then, basically, is to minimize the area below the risk curve, or to suppress its "belly" as much as (practicably) achievable. This is illustrated by Figure 2-2, which also shows the two basic and most obvious principles of the risk/safety management. The third principle, which is not explicitly described in the picture but is mostly used in the practice, represents combination of the two.

Ensuring low consequences by system design (principle a) is achieved through demonstration of the safety margins, which is a deterministic discipline and is implemented as a part of system's design basis analyses. On the other hand, ensuring low likelihood of scenarios which may exceed the design basis and, hence, result in large consequences is done considering the results of probabilistic risk analyses. Deterministic design basis analyses and probabilistic risk analyses, therefore, are considered in ensuring the overall NPP safety and play complementary roles in the plant design and operation.

Safety importance of a particular component or feature within a system or plant also reflects these two aspects (or two dimensions, with respect to Figure 2-1 and Figure 2-2). The first one is importance with 
4 Risk Importance Measures in the Design and Operation of NPPs

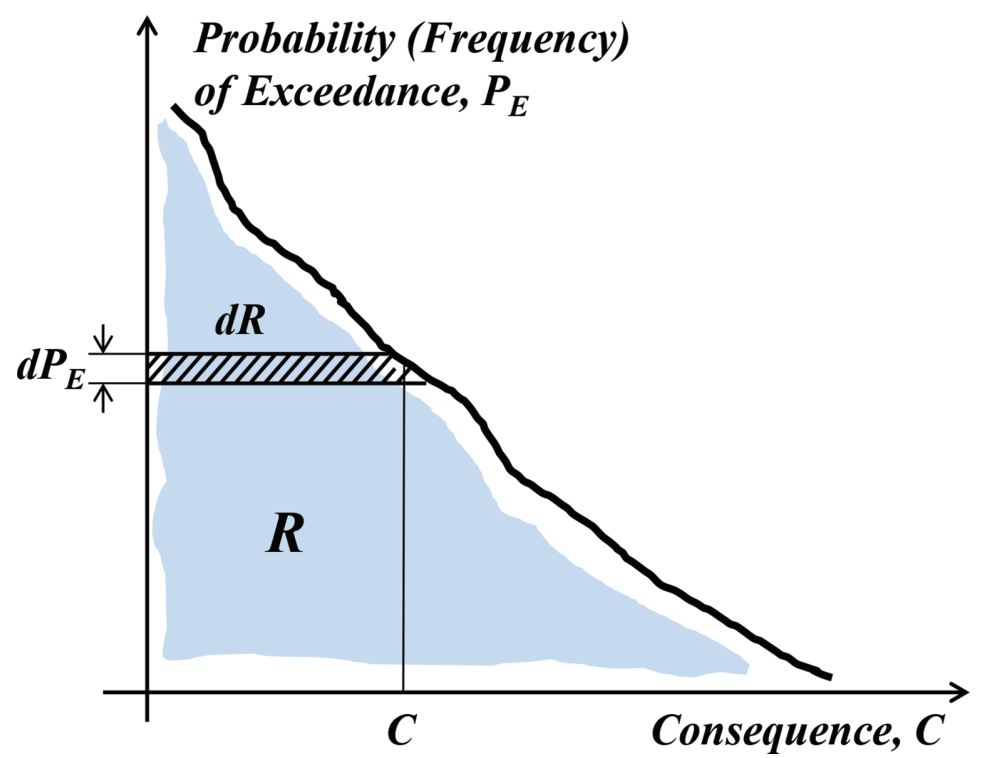

Figure 2-1 Risk curve or definition of risk for an engineer.

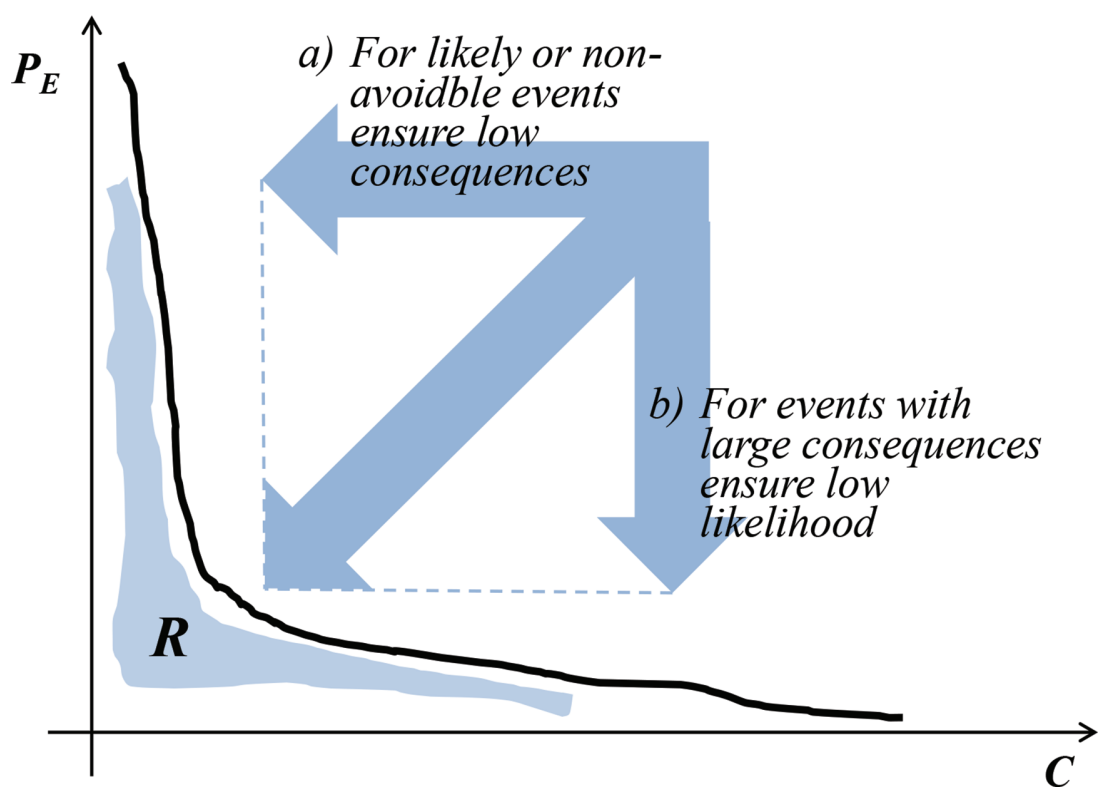

Figure 2-2 Two basic principles of safety/risk management. 
respect to the role in ensuring the appropriate safety margin within the design basis envelope. This importance is addressed deterministically by employing the principles of design bases and defense-in-depth reflected in "traditional" safety classification of equipment. The second aspect is addressed considering importance with respect to the risk, or risk importance, with regard to those scenarios which can lead to the conditions beyond design basis envelope. Generally, this is risk importance which is the subject of the discussions in this monograph. 


\section{Deterministic principles of safety importance and need for consideration of risk}

Traditional view on "importance" of a particular SSC was associated with its role (function) in ensuring appropriate safety margins (and, hence, low undesirable consequences) in the case of anticipated or credible occurrences. The "importance" of an SSC was related to its quality requirements resulting in a set of deterministic rules and principles for the classification of SSCs into quality groups such as " $\mathrm{A}$ ", "B", "C" and "D" ("Class 1", "Class 2", "Class 3" and "Non-Class"). For example, the highest importance with regard to ensuring all safety margins concerning the cooling of the reactor core was given to the integrity of the primary system. Hence, the components of the reactor coolant pressure boundary were assigned Quality Group "A" (or "Class 1"). In the case of primary system integrity failure, ensuring the safety margins relies on the systems with functions such as emergency core cooling and containment heat removal. Thus, those systems were assigned Group "B" (or "Class 2"); supporting systems, such as cooling water systems, to the systems of Group "B" were assigned Group "C" (or "Class 3"), etc.

Taking fluid systems as examples, USNRC Regulatory Guide 1.70 [2] requires that Section 3.2.2 of Safety Analysis Report (SAR) identifies those fluid systems or portions of fluid systems important to safety together with the industry codes and standards applicable to each pressure-retaining component in these systems. It refers to $10 \mathrm{CFR}$ 50.55 as specifying the quality requirements for the reactor coolant pressure boundary, and to Regulatory Guide 1.26 for describing a quality group classification system and relating it to industry codes for water and steam-containing fluid systems. Mentioned Section 3.2.2 of SAR should contain group classification boundaries of each safety-related system. The classifications are noted for valves or other appropriate locations in each fluid system where the respective classification changes in terms of the NRC group classification letters, for example, from A to B, $\mathrm{B}$ to $\mathrm{C}, \mathrm{C}$ to $\mathrm{D}$, as well as other combinations (or alternately, in terms of corresponding classification notations that can be referenced with those classification groups in Regulatory Guide 1.26).

Mentioned Regulatory Guide in its Revision 1 [3] clarified, after reviewing a number of applications for construction permits and operating licenses and after discussions with representatives of professional 


\section{Risk Importance Measures in the Design and Operation of NPPs}

societies and industry, the quality classification system for safety-related components containing radioactive material, water, or steam in watercooled NPPs. The quality classification system consists of four quality groups, A through D, methods for assigning components to these quality groups, and the specific quality standards applicable to each quality group. The initial, portion of this system was described in mentioned $\$ 50.55 \mathrm{a}$ of 10 CFR Part 50, which required that components of the reactor coolant pressure boundary be designed, fabricated, erected, and tested to the highest available national standards, which corresponded to the quality standard required for quality Group A of the regulatory system. Regulatory Guide 1.26 (Revision 1) described a method for determining acceptable quality standards for the remaining safety-related components containing radioactive material, water, or steam, i.e., quality Group B, C, and D components. Other systems not covered by this guide (i.e. Regulatory Guide 1.26), such as instrument and service air, diesel engine and its generators and auxiliary support systems, diesel fuel, emergency and normal ventilation, fuel handling, and fire protection systems using other than water, should be designed, fabricated, erected, and tested to quality standards commensurate with the safety function to be performed.

In Revision 4 of Regulatory Guide 1.26 [4], it is explained that in 1984 the United States Nuclear Regulatory Commission (USNRC) published a final rule amending 10 CFR 50.55a to incorporate by reference the criteria in Section III of the ASME Code, as they relate to the design and fabrication of Class 2 and 3 components (Quality Group B and $\mathrm{C}$ components, respectively, in the regulatory system). Furthermore, it is stated that, because the quality group classification system is wellestablished, the Revision 4 of Regulatory Guide 1.26 retains the method described in previous versions for determining acceptable quality standards for Quality Group B, C, and D components.

Thus, current deterministic framework for safety classification of fluid systems, reflecting their safety importance, is provided by 10 CFR 50.55a [5], and mentioned Revision 4 of Regulatory Guide 1.26, with referenced codes and standards.

The consideration of importance of components is also seen in the USNRC's Standard review Plan (SRP). SRP Section 3.2.2 [6], emphasizes that NPP systems and components important to safety should be designed, fabricated, erected, and tested to quality standards commensurate with the importance of the safety function to be performed. It states 
that the approach to classify fluid systems important to safety is primarily deterministic. The key principle in this deterministic approach to establish the SSC's importance is safety function intended by design. Therefore, establishing of the importance (and, thus, classification) of individual component can only be done by considering the detailed design basis information which defines the intended safety function.

Mentioned SRP Section 3.2.2, recognizing that SAR classification tables typically do not include this kind of information for individual components, directs the reviewers to use the NEI 97-04 Appendix B [7], referenced in RG 1.186 [8], as a possible source to be used to define design basis information regarding the specific safety function in the SAR. NEI 97-04 gives examples of design bases information and was specifically developed to help utilities organize and collate design bases information and supporting design information.

As can be seen from this brief overview, deterministic approach toward definition of importance of SSC mostly relies on its design basis (DB) function, which is usually associated with preserving safety margins under the design basis envelope and, as such, with the principle marked as "a)" in Figure 2-2. Although establishing the safety functions and corresponding design bases does not directly include consideration of risk, classification of components in different categories shows the implicit consideration of risk. Supplementing the deterministic considerations in NPP safety with quantitative characterization of risk importance has been increasingly recognized over the past two or three decades. Some of the reasons relating to consideration of SSC importance are as follows:

+ DB function is many times defined for a limiting scenario. Beside the "main" safety function, many SSCs play roles in mitigation of other types of scenarios, following the failures of other "main" functions. This may not be transparent in DB analyses and is many times revealed through PRAs.

+ "Non-safety" ("non-class") SSCs may play an important role in mitigating some initiators followed by failures of "safety" SSCs. This role may not be recognized in traditional, deterministic, classification but its importance was shown in many PRAs. (In PRAs, one can see many risk-contributing sequences where beyond-DB status can be re-directed back to the DB status by means of "non-safety" systems.) 
- Traditional, deterministic, approach may overlook importance of some operator's actions which are not credited as a part of DB in SARs, but are normally included in the emergency or abnormal operating procedures. Those actions can significantly influence the importance of certain SSCs.

+ Traditional approach to classification was built upon "single failure criterion", which was "conservative" in assuming that one train would fail. However, what is many times neglected is that this approach takes for granted that the other train(s) would operate. In other words, the importance of CCF can be masked, which was demonstrated by many PRAs.

These, and many other, reasons point to the different levels of importance which certain ("safety" and "non-safety") SSCs may have with respect to the principle depicted in Figure 1-2 marked as "b)". While focusing on the importance of preserving safety margins within $\mathrm{DB}$ envelope, the importance of ensuring low likelihood of scenarios beyond DB may be overlooked. This is recognized in SRP Section 3.2.2 [6], which points out that "the deterministic approach should be complemented, where appropriate, by applying insights from the design-specific probabilistic risk assessment". The same recognition is also reflected in a number of risk-informed applications making use of risk importance measures, some of which we discuss in Section 4.3. 


\section{Risk importance measures in NPP risk assessment}

\subsection{Background on importance measures in risk assessment}

It can be said that the idea for risk importance measures originates from the concept of traditional sensitivity analysis, which might not necessarily be related to the risk (reliability) studies. One type of sensitivity analysis is to change one of the parameters and see how much effect it makes on the result [9]. A systematic approach would be to calculate the fractional change in result for a fractional change in a variable or parameter. As an example ([9]), suppose the risk, $R$, is a function of $n$ variables:

$$
R=R\left(P_{1}, \ldots, P_{n}\right)
$$

The total derivative is

$$
d R=\frac{\partial R}{\partial P_{1}} d P_{1}+\ldots+\frac{\partial R}{\partial P_{n}} d P_{n}
$$

Partial derivative $\frac{\partial R}{\partial P_{1}}$ appears to be a perfectly good sensitivity measure. However, it suffers a significant liability in that given absolute change in variable $d P_{1}$ may be a very large or very small fractional change, depending on the range of values the variable can take. It seems, then, to be much better to assess the fractional changes, which can be done by taking the logarithmic derivative [9]:

$$
d(\ln R)=\left[\frac{\partial(\ln R)}{\partial\left(\ln P_{1}\right)}\right] d\left(\ln P_{1}\right)+\ldots+\left[\frac{\partial(\ln R)}{\partial\left(\ln P_{n}\right)}\right] d\left(\ln P_{n}\right)
$$

or

$$
\frac{d R}{R}=\left[\frac{\partial(\ln R)}{\partial\left(\ln P_{1}\right)}\right] \frac{d P_{1}}{P_{1}}+\ldots+\left[\frac{\partial(\ln R)}{\partial\left(\ln P_{n}\right)}\right] \frac{d P_{n}}{P_{n}} .
$$

The term in parentheses, $\left[\frac{\partial(\ln R)}{\partial\left(\ln P_{i}\right)}\right]=\frac{\partial R / R}{\partial P_{i} / P_{i}}$, represents the sensitivity of a fractional change in the result to a fractional change in the variable 
and is, according to [9], the commonly understood meaning of "sensitivity". The same reference uses this term as a definition of Fussell-Vesely (F-V) importance measure, which is one of the basic risk importance measures in today's PRAs. Indeed, if we, for simplicity, assume that $R$ is a linear function of $P_{i}$ (all other variables' values fixed), $R=k P_{i}+l$, the above term becomes

$$
\frac{\partial R / R}{\partial P_{i} / P_{i}}=\frac{P_{i}}{R} \frac{\partial R}{\partial P_{i}}=\frac{k P_{i}}{R}
$$

which can easily be related to the F-V, or "fractional contribution", as it is discussed later in this monograph. (See expressions (5-10) and (5-12) and consider $P_{i}=P(A), k=P(C)$ and $R=P(B)$.)

There are a number of other importance measures which were defined and used in reliability and risk analyses. Some of them are defined in relative and some in absolute terms. Most of them are related to each other and some of them produce same risk ranking. Their theory and use is described in a number of books such as [9], [10] or [11] and studies or engineer's handbooks and guidelines such as [12], [13] or [14]. In this monograph, however, we want to focus on those which are most widely used in current PRAs for NPPs. As an example, ASME PRA Standard of 2009 [15], explicitly defines only two importance measures: F-V and risk achievement worth. One apparent reason is that other relevant importance measures can be related to these two. But, before proceeding to these matters we will first identify a group of importance measures most commonly used in NPP PRAs.

\subsection{Importance measures broadly used in PRAs for NPPs}

In PRA models and applications associated with NPPs and implemented by tools such as RiskSpectrum ${ }^{\circledR}$ and others, the risk importance of a particular component or a failure mode can, most generally, be divided in two categories: importance with respect to risk increase potential and importance with respect to risk decrease potential. In this monograph we explicitly address four importance measures, related to both categories, which can be considered as most broadly used in the current PRAs and their practical applications. 
We will use their definitions from NUREG/CR-3385 [12], which we consider to be one of the references to establish the use of risk importance measures in PRA applications. Let:

$R_{0}=$ the present ("nominal") risk level;

$R_{i}^{+}=$the increased risk level with feature " $i$ " assumed failed;

$R_{i}^{-}=$the decreased risk level with feature "i" assumed to be perfectly reliable.

The first importance measure, risk achievement worth (RAW), is related to risk increase potential. It is defined as ratio:

$$
R A W_{i}=\frac{R_{i}^{+}}{R_{0}}
$$

(Beside ratio, NUREG/CR-3385 also defines the RAW on an interval scale as $R_{i}^{+}-R_{0}$. These two values are related to each other. When one is known, the other can be calculated directly (considering that nominal risk $R_{0}$ would normally be known).)

The second one is related to risk decrease potential. It is risk reduction worth (RRW), defined as:

$$
R R W_{i}=\frac{R_{0}}{R_{i}^{-}}
$$

(In the similar fashion, NUREG/CR-3385 also introduces the related RRW on an interval scale, as $R_{0}-R_{i}^{-}$.)

The third measure is also related to risk decrease potential. NUREG/ CR-3385 refers to it as the fractional contribution (FC) of feature " $i$ " to risk, or the Fussel-Vesely measure (F-V) of feature " $i$, defined as:

$$
F C_{i}=\frac{R_{0}-R_{i}^{-}}{R_{0}}
$$

(It should be noted that, although NUREG/CR-3385 does not formally differentiate between the fractional contribution and F-V, some of the later PRA practices and tools introduced a distinction between the two measures. This distinction is discussed in Section 5.3.) 
In theoretical considerations with associated demonstrating examples presented in this monograph (starting with Section 5), we will use the expression "fractional contribution" for the simple reason that it is descriptive, i.e. the meaning can directly be associated with the measure.

It can be seen from the definitions that RRW is related to FC and that the two measures will produce the same risk ranking. Both of them are included in the considerations to follow, for the sake of completeness.

Beside the above three, there is an additional measure which will be explicitly addressed. It is related to both categories, risk increase and risk decrease. It measures how the risk $R$ changes with change in component's failure probability $p_{i}$. For this measure, the name "reliability importance" and the general definition was taken from Barlow and Proschan [11]:

$$
I_{r e l, i}=\frac{\partial R}{\partial p_{i}}
$$

As shown later (section 5.6), this measure can be related to the above measures through: $I_{r e l, i}=R_{i}^{+}-R_{i}^{-}$. When written in this form, it is often referred to as "Birnbaum importance" (e.g. the mentioned NUREG/ CR-3385 [12]).

\subsection{Examples of use of risk importance measures in risk-informed applications}

Many risk-informed applications have been carried out using PRAs of NPPs to improve operational safety and bring improved focus on regulatory requirements while at the same time reducing regulatory burden and operational cost. These applications are usually associated with specific uses of the risk-importance measures discussed above. In this section, we will present some examples on the use of risk-importance measures in risk-informed applications to provide a perspective on how the importance measures are the basic building blocks in these applications.

We will present a summary discussion of four different risk-informed applications that have been broadly used by nuclear operators and regulators:

1. Risk-informed categorization of and treatment of SSCs for nuclear power reactors

2. Risk-informed changes to NPP Technical Specifications 


\section{Application of Maintenance Rule \\ 4. Applications associated with Reactor Oversight Process (ROP).}

They are discussed in the four separate subsections below.

\subsubsection{Risk-informed categorization and treatment of SSCs for nuclear power reactors}

This application allows adjustment to the scope of equipment subject to special regulatory treatment (controls) to better focus regulator and plant operator's attention on equipment with safety significance. U.S. NRC promulgated a rule, 10 CFR 50.69 [16], defining the process and our discussion uses the application of this rule to present the example.

Prior to 10 CFR 50.69, NRC's deterministic regulations were used to categorize plant equipment as "safety-related" or "non-safety-related" (Refer, also, to Section 3.) The "safety-related" equipment is subject to NRC's special treatment regulations whereas "non-safety-related" equipment is not. There is a set of "non-safety-related" equipment that is subject to a selected number of special treatment requirements or a subset of those requirements. This third category of equipment is referred to as "important-to-safety."

In this application, risk-informed analyses are conducted to categorize the plant equipment in four categories:

1. Safety-related SSCs that are identified as safety-significant (RISC-1)

2. Some non-safety-related equipment that are identified as safetysignificant (RISC-2)

3. Some safety-related equipment that are identified as low or of no safety significance (RISC-3)

4. Remaining non-safety-related equipment of low or no safety significance (RISC-4).

This categorization is achieved using PRA, traditional engineering analyses, and consideration of other regulatory requirements. With such a categorization, improved safety in plant operation can be achieved while reducing the regulatory burden. In such an application, it is expected that in many cases the number of SSCs or the regulatory burden associated with the "non-safety-related" equipment defined as RISC-2 will 
be less than the number or the regulatory burden associated with the "safety-related" equipment defined as RISC-3.

The categorization process defined in 10 CFR 50.69 requires consideration of the following:

1. Use of results and insights from a plant-specific PRA that includes determination of SSC functional importance using an integrated, systematic process for addressing initiating events (internal and external), SSCs, and plant operating modes, including those not modeled in the plant-specific PRA

2. Maintaining defense-in-depth

3. Evaluations that provide reasonable confidence that for SSCs categorized a RISC-3, sufficient safety margins are maintained and that any potential increases in core damage frequency (CDF) and large early release frequency (LERF) resulting from the changes are small

4. Performing for entire systems and structures; not for selected components within a system or structure

5. Using an Integrated Decision-Making Panel (IDP) staffed with expert, plant-knowledgeable members whose expertise includes, at a minimum, PRA, safety analysis, plant operation, design engineering, and system engineering.

Full details of the considerations involved can be obtained from 10 CFR 50.69 and in NEI 00-04, Rev. 0 [17].

NEI 00-04 defines a process for identifying the safety significance of a component. The process includes two risk-importance measures, discussed above, as screening tools to identify candidate safety-significant SSCs:

+ RAW, and

+ F-V.

(RRW is also considered an acceptable measure in place of F-V "because the F-V criteria can be readily converted to RRW criteria".)

The RAW importance of a component is considered the maximum of the RAW values computed for basic events involving the failure modes of the individual component. To address the component's contribution 
to common-cause failures, if applicable, a separate assessment of the common cause basic events in which the component is included is conducted. The RAW for common cause basic events reflects the relative increase in CDF/LERF if the set of components included in the common cause event is made unavailable. The risk-significance of the RAW values of common cause basic events is considered separately from the basic events that represent an individual component. Similarly, if the component is included in more than one common cause basic event, the maximum of the common cause RAW values is used to evaluate the significance.

The F-V importance of a component is considered to be the sum of the F-V importance of the failure modes of the component relevant to the measure (CDF or LERF) being addressed.

The importance measure criteria used to identify candidate safety significance are:

+ Sum of F-V for all basic events modeling the SSC of interest, including common-cause events $>0.005$;

+ Maximum of component basic event RAW values $>2$;

+ Maximum of applicable common cause basic events RAW values $>20$.

If any of those criteria are exceeded for a component, then the component is considered safety-significant.

If the risk importance measure criteria are not met, then it is not automatically low safety significance. Additional sensitivity evaluations are conducted for assessing it to be low safety significant for all contributors and are reviewed by the IDP. If the sensitivity studies identify that the component can be safety-significant, then the safety-significant attributes that yielded that conclusion are identified. Sensitivity studies are conducted with respect to human error rates, CCFs, and maintenance unavailabilities to ensure that the assumptions of the PRA are not masking the importance of an SSC. Details of the sensitivity studies can be obtained in NEI 00-04.

The evaluations are carried out for both CDF and LERF. NEI 00-04 discusses the considerations for truncation levels in conducting these evaluations. It is stated that when the RAW risk importance measure is calculated by a full resolution of the plant PRA model, then the truncation 
level does not significantly affect the RAW calculations. (Here, it is worth noting that many PRA practitioners actually use very low cut off and produce much more minimal cut sets (MCSs) for the purpose of RAW calculations than for the base case top event evaluation.) NEI 00-04 provides recommendations on how to address an impact of truncation level on calculated importance measures both for PRAs using linked fault trees (FT) and those using linked event trees (ET).

\subsubsection{Risk-informed changes to NPP technical specifications}

Technical Specifications (TSs) for NPPs define the limits and conditions to safe operation consistent with the analyses and evaluations in the plant's safety analysis report. TS requirements include Limiting Conditions for Operations (LCOs) that define Conditions, Required Actions, and Completion Times (CTs); and Surveillance Requirements (SRs). A broad spectrum of assessments and experience are used to define and assess changes to these requirements that involve deterministic analyses, knowledge of lessons learned from previous changes, engineering judgments, and risk implications of the requirements.

PRAs are used to define measures that are used to quantitatively assess the risk associated with these requirements and they form a useful input in defining and evaluating changes to these requirements. These requirements which were originally based largely on engineering judgments have been improved using risk measures and risk-informed analyses.

Two of the TS requirements: CT and Surveillance Frequency (SF) are evaluated using risk-informed analyses in terms of risk measures. CT defines the time for which a component or a train in a safety system can remain inoperable before an action is required, which is typically a plant shutdown. A CT is used to repair or replace a failed or a degraded component, and sometimes, also to carry out a scheduled maintenance. SF defines the frequency of surveillance testing which is required to assure that the components of standby safety systems will be operable when they are needed in case of an accident. By testing these components, failures are detected that may have occurred since the last test or the last time the component was last known to be operational.

\subsubsection{CT risks}

Two types of CT risks are measured to evaluate the risks associated with a CT: single-event CT risk and yearly CT risk. The single-event 
CT risk is the risk associated with a given occurrence of the CT. The yearly CT risk contribution is the average yearly risk contribution from the $\mathrm{CT}$, accounting for its yearly average frequency.

The single-event CT risk, in terms of core damage frequency, is the probability of a core damage occurring during the period the component is down. It is mathematically expressed as:

$$
r=\Delta R d
$$

where,

$r=$ the single-event CT risk,

$\Delta R=$ the increase in the conditional risk level, given that the component is down, and

$d=$ the downtime associated with the CT.

The yearly CT risk is the product of the single-event CT risk and the frequency of the occurrence of the CT:

$$
R_{y}=f r=f \Delta R d
$$

where,

$f=$ the average yearly frequency of occurrences of the CT or the downtime frequency, and

$R_{y}=$ the yearly CT risk contribution.

Typically, these two measures are used to define or to decide on changes to the CT in the TS.

PRA is used to calculate the increase in the risk level $\Delta R$ :

$$
\Delta R=R^{+}-R^{-}
$$

where,

$R^{+}=$the increased CDF level when the component is known to be down or unavailable, and

$R^{-}=$the reduced CDF level when the component is known to be up or available.

So:

$$
r=\left(R^{+}-R^{-}\right) d
$$


and

$$
R_{y}=f\left(R^{+}-R^{-}\right) d
$$

As already mentioned in 4.2, the term $\left(R^{+}-R^{-}\right)$represents the reliability (or the Birnbaum) importance measure. The terms $R^{+}$and $R^{-}$ can, also, be obtained from the RAW and RRW (or F-V) importance measures.

Thus, with the availability of Birnbaum importance or RAW and RRW (or F-V), the above discussed two measures can be calculated for evaluating the CT in TS.

\subsubsection{SF risks}

Surveillance tests detect failures that may have occurred between tests. At the same time, the test may have some adverse impacts on safety because of their undesirable effects, e.g., test errors causing plant transients, wear out of equipment caused by testing, downtime associated with the test. Thus, SF is broadly associated with two types of risks: (a) risk detected by the test and (b) risk-caused by the test. We will discuss here the risk detected by the test, sometimes called the test-limited risk, since it is associated with the risk importance measures.

The risk detected by a surveillance test (test-limited risk contribution), $R_{D}$, can be written as:

$$
R_{D}=q \Delta R \approx \frac{\lambda T}{2}\left(R^{+}-R^{-}\right)
$$

where,

$q=$ probability of failure of a standby component on demand $\left(\approx \frac{\lambda T}{2}\right)$,
$\lambda=$ the component failure rate per unit time,

$T=$ the surveillance test interval.

Here again, the SF risk contribution is directly related to the reliability (Birnbaum) importance and also can be derived from the RAW and RRW.

These risk measures of CT and SF have been used to revise CT and SF in plant-specific TS and NRC Regulatory Guide RG 1.177 [18] provides guidance for making changes to these requirements in riskinformed analyses. 
Additionally, it is worth mentioning that risk evaluations which draw more and more attention are those aiming at comparison of the risk of shutdown to the risk of continuing the operation and repairing the equipment.

\subsubsection{Application in Maintenance Rule}

Effective maintenance of system, structures, and equipment in an NPP has a clear link to safety and as discussed in the U.S. NRC's Maintenance Rule 10 CFR 50.65 [19], it plays many safety enhancing roles:

+ Improves such factors as number of transients, challenges to safety systems, and associated need for operability, availability, and reliability of safety equipment,

- Minimizes failures of other than safety-related SSCs that could initiate or adversely affect a transient or accident, and

+ Proves assurance that design assumptions and margins in the original design basis are either maintained or are not unacceptably degraded.

The NRC's Maintenance Rule (MR) provides regulatory framework for assessing the effectiveness of an NPP's maintenance program. The MR is considered performance-based, risk-informed rule and allows consideration of risk in selecting and monitoring of SSCs for the rule. Paragraph (a)(1) of the MR requires licensees to monitor the performance or conditions of SSCs and to establish goals to promote improved SSC performance, when warranted. Paragraph (a)(2) of the rule states that monitoring pursuant to paragraph $(\mathrm{a})(1)$ is not required for SSCs that are being effectively maintained by a preventive maintenance program. Paragraph (a)(3) of the rule requires that performanceand condition-monitoring activities and associated goals and preventive maintenance activities be evaluated at least every refueling cycle (not to exceed an interval of 24 months), taking industry-wide operating experience into account, where practical. Paragraph (a)(4) states that licensee shall assess and manage the increase in risk that may result from proposed maintenance activities.

Regulatory Guide 1.160 [20], and NUMARC 93-01 [21], provide guidance for implementing the MR. In these documents, risk importance measures are used for determining which SSCs that are under 
the scope of the MR will have goals and monitoring established in accordance with (a)(1).

NUMARC 93-01 calls for use of following three measures for identifying the SSCs:

+ RRW,

+ RAW, and

+ CDF Contribution.

SSCs selected by use of these measures are then provided to the expert panel for finalizing the SSCs to be monitored under (a)(1).

The following criteria are used in the NUMARC 93-01:

\section{RRW}

Two alternative methods are provided for applying RRW in identifying the risk significant SSCs.

An SSC is considered risk significant if its risk reduction importance measure contributes to at least 99.0 percent of the cumulative risk reduction importance.

Alternatively, an SSC is considered risk significant if its RRW exceeds $0.5 \%$ of the overall CDF (RRW>1.005).

2. RAW

An SSC is considered risk significant if its RAW shows at least a doubling, i.e. RAW > 2.0, of the overall CDF.

3. CDF Contribution

An SSC is considered risk significant if it is included in cut sets that, when ranked in decreasing order, cumulatively account for about 90 percent of the CDF.

The MR became effective for U.S. NPPs in 1996 and many consider that the rule have contributed to industry's significantly improved performance by focusing attention and corrective actions on those SSCs that exhibited poor performance. In general, the rule is considered to have improved operating and maintenance practices across the industry.

\subsubsection{Applications associated with ROP}

The U.S. NRC's ROP is the agency's program to inspect, measure, and asses the safety performance of NPPs and to respond to any decline in performance, NUREG-1649 [22]. A major element of this oversight 
program is to focus inspections on activities where the potential risks are greater. It also calls for responding to violations of regulations in a predictable and consistent manner that reflects the potential safety impact of the violations.

The framework of the ROP defines three key areas: Reactor safety, Radiation safety, and Safeguards. It also defines seven cornerstones and three cross-cutting areas. To discuss the use of the risk importance measures, we will focus on the reactor safety area which is associated with four cornerstones: initiating events, mitigating systems, barrier integrity, and emergency preparedness. The cross-cutting areas associated with all the cornerstones are: human performance, safety conscious work environment, and problem identification and resolution.

To focus inspection on activities where the potential risks are greater, each of the cornerstones is analyzed in terms of key attributes of licensee performance contributing to the cornerstone. For example, for the initiating event cornerstone, the key attributes are: design, protection against external events, configuration control, equipment performance, procedure quality, and human performance. Each of these key attributes is then studied to define the areas and means to measure. Risk-informed inspection is defined as the means to measure in many cases. Based on these analyses, areas for inspection are defined for which inspection procedures are developed.

For the Reactor safety area and the associated cornerstones of initiating events, mitigating systems, and barrier integrity, seventeen areas for inspection are defined. Inspection Procedure 71111 under NRC Inspection Manual [23], presents these procedures. The examples of areas for inspection in these cornerstones are: adverse weather protection, equipment alignment, fire protection, maintenance effectiveness, component design bases inspection, and surveillance testing.

Inspection guidance is provided for conducting inspection in each of the areas. The inspection guidance includes risk-informed inspection planning. The risk-informed inspection planning involves selection of risk-informed inspection samples which is based on the following;

+ Extracting risk insight from a risk model;

+ Using these insights to select SSCs and activities for inspection; and

+ Using insights from plant-specific and industry operations experience to add SSCs into the inspection sample. 
Plant-specific PRAs are used to extract risk-insights and to obtain items for inspection. The types of information that are used are the following:

+ List of dominant accident sequences and their contribution to CDF and LERF,

+ List of accident initiators, components, systems, and operator actions ranked by risk importance measures:

+ RAW,

+ RRW,

+ Birnbaum, and

$+\mathrm{F}-\mathrm{V}$,

+ List of potential accident sequence vulnerabilities.

Other items, as applicable, can be considered by the inspectors in finalizing the areas and items for inspection.

The consideration of the risk insights and risk-importance measures in defining the focus of inspection provides the desired emphasis on activities where the potential risks are greater and thereby making the regulatory inspection effective, contributing to safe operation of the NPP.

System performance indicators is another example of an application of risk importance measures within the ROP framework. In its ROP, the NRC used performance indicators to quantify safety system unavailability [24], which gave way to the development of a more riskinformed performance indicator known as the Mitigating Systems Performance Index (MSPI). The MSPI, in its essence, represents a change in plant risk (measured through CDF) due to a change in system's availability or reliability. By controlling the MSPI, a decline in availability or reliability can effectively be controlled. The MSPI, as a change in plant risk due to a change in system's availability or reliability, is assessed through the risk importance measures. As an example, the unavailability-related contribution to MSPI from a particular train can be written as ([24], [25]):

$$
\Delta C D F_{t, U}=U A I_{t}=I_{r e l, t, U} \Delta U_{t}
$$

where

$\Delta C D F_{t, U}=$ change in core damage risk due to a change in train " $t$ " unavailability;

$U A I_{t}=$ train " $t$ " unavailability index; 

$I_{r e l, t, U}=$ Birnbaum importance measure (or "reliability importance", as referred to in the remaining part of this monograph) for the train " $t$ " unavailability;
$\Delta U_{t}=$ measured (i.e. calculated from operating performance) change in unavailability of train " $t$ ".

The Birnbaum importance can further be developed into ([24], [25]):

$$
I_{r e l, t, U}=\mathrm{CDF}_{p} \frac{F C_{t, U, p}}{U_{t, p}}
$$

where

$$
\begin{aligned}
\mathrm{FC}_{t, \mathrm{U}, p}= & \text { fractional contribution, or Fussel-Vesely, importance } \\
& \text { measure for unavailability of train " } t \text { ", calculated from } \\
& \text { PRA; } \\
U_{t, p}= & \text { unavailability of train " } t \text { ", from PRA; } \\
\mathrm{CDF}_{p}= & \text { core damage risk as calculated by PRA model. }
\end{aligned}
$$

For the relation of the reliability importance (Birnbaum importance) to other importance measures, refer to Section 5.6.

\subsection{Risk importance measures focus of discussion in this monograph}

Having in mind the considerations in the preceding sections, the discussions in the remainder of this monograph will focus on the four risk importance measures:

+ RAW;

+ RRW;

- Fractional contribution (Fussel-Vesely importance);

+ Reliability importance.

The risk importance measures which are directly reported by PRA tools relate, in most cases, to basic events (representing particular failure modes or human errors). On the other hand, what is in engineering practice really needed is risk importance of actual components (e.g. pumps or valves). It is, therefore, desirable to find a way to express the "component-level importance measures" for the components represented in a model. 
In PRAs, components are modeled in terms of their failure modes which are represented by the basic events associated with a component in the model. When a component is involved in a common-cause failure group, i.e., a common-cause failure is associated with the component and other similar components, then the basic event for the commoncause failure of the components is also represented in the model. In many cases, more than one failure mode of a component is associated with a common-cause failure. Common-cause failure parameters of the failure modes are typically different and are represented by different basic events. Thus, multiple basic events in the model can represent a component failure, some (or all) of them being involved in common cause failure groups.

As presented above, risk importance measures obtained from a PRA model are typically obtained in terms of the basic events. However, in many PRA applications judgments are required for components and not for a specific component failure mode. Additionally, the basic event associated with a common-cause failure provides the risk significance of the common-cause failure group or the failure of the multiple components associated in a common-cause failure. The user then will have to judge the risk significance of the component by looking at the risk-significance of the multiple, different basic events associated with a component. In many cases, judgments are made without consideration of the common-cause failure basic event.

Component-level risk importance measure discussed in this monograph aims at addressing this issue in a way to provide a single measure that takes into account the different failure modes of a component along with its contribution to common-cause failures modeled for the component. This single measure would then fully represent the risk significance of the component and could be directly used in different applications. A list could be generated from the computer codes rank ordering the components in terms of the component-level measures. This would be useful for many applications and makes the process straight-forward facilitating use of PRAs.

General approach taken for establishing any of the considered component-level risk importance measures (e.g. component-level RAW) was to express it as a function of importance measures for the representative basic events which are normally reported by a PRA tool. 
In order to establish analytical expression for component-level risk importance two types of issues need to be addressed:

a. Particular component is in most cases represented by multiple basic events related to different failure modes. Sometimes, the same failure mode is represented by different basic events in different accident sequences (event sequences).

b. Component, or some of its failure modes, may or may not be involved in CCF groups with other components.

Development of component-level risk importance measures addressing above two issues requires dealing with different scenarios that are experienced in NPP PRAs. The following cases are considered to address different failure mode combinations, including commoncause failures, of practical considerations. The cases are presented with increasing complexity considering the four risk importance measures presented at the beginning of this subsection.

a. Component is not involved in any CCF group.

- The simplest case is a component presented by a single basic event. This is discussed in Section 5 which introduces the definitions to be used in all sections to follow and establishes the relations between particular importance measures for a single basic event.

- Many times, a component is in a system's fault tree (FT) presented as a sub-tree, e.g. an "OR" gate with inputs which are basic events representing different failure modes. Similar types of components are many times presented by similar subtrees which are often referred to as "FT modules". Risk importance measures for such component-level "FT modules" are discussed in Section 6.

- Sometimes, the component is presented by different FT modules in different accident (ET) sequences. (It is noted that the simplest FT module is a single basic event.) Risk importance measures for such a case are discussed in Section 7.

b. Component is involved in a CCF group.

- Section 8 discusses risk importance measures for the case when component is presented by a single failure mode (basic event). 
+ Section 9 discusses, under some simplifications, risk importance measures for the case when component is presented by multiple failure modes (basic events) which are involved in CCF groups.

All the cases are accompanied by the demonstrations in the form of examples based on the simplified PRA model. The examples are presented in Appendix B.

The component-level risk importance measures being defined and presented in this monograph and their relevance in terms of the risk importance measures typically calculated in a (current) PRA are illustrated by a simple example as follows.

Let us consider components $\mathrm{A}, \mathrm{B}$, and $\mathrm{C}$ within a PRA model for an NPP. Component A is associated with three basic events: failure to start, "Comp A-FS", failure to run, "Comp A-FR", and test or maintenance unavailability, "Comp A-TM". Similarly, component B is associated with three basic events: failure to start, "Comp B-FS", failure to run, "Comp B-FR", and test or maintenance unavailability, "Comp B-TM". Component $\mathrm{C}$ is also associated with three basic events: failure to open, "Comp C-FO", plugs during operation, "Comp C-PL", and fails to remain open, "Comp C-FRO". Additionally, components A and B are of the same type and there is a potential for a common-cause failure. Thus, there are two CCF basic events: components $\mathrm{A}$ and $\mathrm{B}$ fail to start due to CCF, "Comp AB-FSCC" and components A and B fail to run due to CCF, "Comp AB-FRCC". Also, a human error of restoration is associated with component C, "Comp C-HER". RAW is used to explain the new component-level importance measure being discussed in this monograph. Table 4-1 presents two columns explaining the RAW measures calculated currently in a PRA (column 1) and the additional RAW measures to be discussed here (column 2) to facilitate the use of risk importance measures in many applications as an additional input in the decision-making process.

As shown in the table, to decide on the risk significance of a component, a number of risk importance measures of the basic events associated with component is used to make a judgment. By creating a component-level importance, as shown in the second column, based on the basic event importances associated with the component, the use of risk importance measure becomes more direct and clearer. Considering these component-level importance measures would make the use of risk 
Table 4-1 Example of RAW importance measures for basic events and components.

\begin{tabular}{lc}
\hline $\begin{array}{l}\text { RAW measures for } \\
\text { basic events currently } \\
\text { calculated by a PRA }\end{array}$ & $\begin{array}{c}\text { RAW measures being } \\
\text { additionally discussed } \\
\text { in this monograph }\end{array}$ \\
\hline Comp A-FS & Comp A \\
Comp A-FR & Comp B \\
Comp A-TM & Comp C \\
Comp B-FS & Comp C-HER \\
Comp B-FR & \\
Comp B-TM & \\
Comp AB-FSCC & \\
Comp AB-FRCC & \\
Comp C-FO & \\
Comp C-PL & \\
Comp C-FRO & \\
Comp C-HER & \\
\hline
\end{tabular}

importance measures in PRA applications more straight-forward and less subject to interpretation.

The need for assessing the component-level importance measures has been recognized time ago and was present in the handbooks for PRA applications as well as in PRA-related literature for decades. Thus, for example, NEI 00-04 [17], mentioned in Section 4.3 .1 above, contains a procedure for establishing component-level importance measures based on the importance measures of particular basic events mapped to the component of concern. There is, also, a number of papers published in scientific magazines or conferences' proceedings devoted to the subject.

Thus, for example [26] pointed to and discussed some difficulties and uncertainties associated with this (importance measure for multiple basic events). Then, the paper [27], published in the same year (1998), discussed the subject of calculation of conditional core damage probability (CCDP) for nuclear power plant operation. It discussed, among others, the subject of "adjustment" of common cause failure probabilities given a failure (or unavailability) of a component involved in a CCF group. Strictly speaking, the subject matter of [27] is not calculation of importance measures but, rather, calculation of a CCDP for precursor 
events (initiators or conditions). However, it is relevant for the importance measures since those are related to CCDP (or conditional top event probability), as discussed in the next session. One of the more recent references is paper by Kim et al. of 2005 [28], which discussed calculation of component's RAW involving CCFs. Then, there is one of the most recent references [29], which discusses issues with calculating the RAW (or "risk increase factor") for a component represented by multiple basic events in a PRA model. The same reference also provides an overview of the relevant work on the risk importance measures during past decades. There are, also, other references, some of which are mentioned in the sections which follow when discussing the related matters.

The discussions and considerations of component-level risk importance in this monograph are not meant to be any further research based on mentioned (or any other) references or to evaluate the positions and/ or methods described in them. They are, rather, meant to describe the principles for calculations of risk importance measures for single basic events and for components represented by multiple basic events rooted in probability theory, which is the basis for any PRA quantification. In any case, an interested reader is welcome to further explore the mentioned references for additional insights into specific aspects and difficulties associated with assessing component-level importance measures.

Additionally, there are references such as [30] which discuss practical aspects of applying risk importance measures in NPP operation. Interested readers are invited to read those for insights concerning the interpretation of RAW and FC (or F-V) in practical risk-informed applications such as use of PRA to support the demonstration of effectiveness of SSC maintenance programs, configuration risk monitoring or categorization and treatment of SSCs. 


\section{Risk importance of component represented by a single basic event not involved in a CCF Group}

\subsection{Introductory considerations}

At the beginning, we introduce two basic terms which will be used in all considerations to come. Both of them are "events":

A Failure of component by a considered failure mode, when challenged. (This failure mode is presented in a PRA model by specifically defined single basic event.)

$B$ Occurrence of specified top event representing certain damage state (e.g. reactor core damage).

The probability of top event $B$, i.e. $P(B)$, will be taken as a surrogate for the quantitative risk $R$ which was used in general expressions for importance measures in 4.2. This is normally done in PRA models. One issue with this is that some of the most important quantitative risk surrogates in PRA are expressed as frequencies rather than probabilities (e.g. $\mathrm{CDF}$ ). The frequency is brought into the risk equation by initiators. We will "bypass" the above issue, without going into mathematical formalism, by considering that in PRAs the initiators (at least those expressed in terms of frequency) are defined as mutually exclusive events. Due to this the risk equation can be written in the general form of (numerical) sum:

$$
f_{B}=\sum_{j} f_{I E, j} Q_{j}
$$

where $f_{B}, f_{I E, j}, Q_{j}$ refer to frequency of top event $B$, frequency of initiator " $j$ " and corresponding conditional probability for leading to the occurrence of $B$, respectively. Both sides of the above equation can be multiplied by an arbitrary small time interval $\Delta T$ such that $f_{I E, j} \Delta T<<1$ (and, hence, $f_{B} \Delta T<<1$ ), enabling, thus, products $f_{B} \Delta T$ and $f_{I E, j} \Delta T$ to be interpreted as probabilities (of occurrence within the time interval $\Delta T$ ). For example, initiator frequencies (and, hence, CDF result) in a PRA model are usually expressed on per year basis. Selecting $\Delta T=\frac{1}{8760}$ year (which, basically, translates the values to the per hour 
basis - which is, sometimes done for risk-monitoring purposes) would satisfy the above condition for all typical initiator categories (considering, e.g., the frequencies from NUREG/CR-5750 [31]). Once any calculation of an importance measure is completed, the result can, if needed, be divided by the same $\Delta T$ and converted to the "per year" basis. (This may be, for example, relevant for the reliability importance. It is not relevant for the measures which are calculated as ratios.) Of course, the whole above process (multiplying and dividing) is only a mental exercise and is here presented pro forma.

In the considerations and discussions which follow below, as well as in the remaining part of the monograph, the following convention was used for simplicity: if $x$ and $y$ present logic variables (events or statements) then the term $x+y$ presents logic sum (union) and the term $x y$ presents logic product (intersection); if $x$ and $y$ present numeric variables then the term $x+y$ presents numeric sum and the term $x y$ presents numeric product.

It will be, in further considerations, assumed that top event $B$ (e.g. reactor core damage) can be expressed in the form of logical sum of minimal combinations of failures, i.e.:

$$
B=\sum_{x=1}^{n} M_{x}
$$

where particular $M_{x}$ represents minimal combination of failures (including the initiator) required for the top event to occur:

$$
M_{x}=M_{x, 1} M_{x, 2 \ldots} M_{x, m}
$$

In the above expression the term $M_{x, i}$ is a basic event representing particular failure. (One of the terms $M_{x, i}$ represents occurrence of an initiator.) The term on the right hand side represents minimum combination of failures which is sufficient for a top event to occur (i.e. "minimal cutset"). (In other words, if any of these failures is removed or prevented, the top event would not occur.)

Let us, further, define:

C Occurrence of any combination of failures following any initiator (and including the initiator), such that the combination 
leads to a challenge to component with regards to the considered failure mode $A$, while successful operation of the component is required in order to prevent the top event (e.g. core damage occurrence); successful operation of considered component is, assuming this combination of failures, sufficient to prevent top event occurrence.

For example, if we identify all minimal combinations $M_{x_{A}}\left(x_{A}=1, \ldots, k\right)$ in the above expression (5-1) which include the failure mode (basic event) $A$, each one of them can be written as: $M_{x_{A}}=A C_{x_{A}}\left(x_{A}=1, \ldots, k\right)$ and the term $C$ would then be:

$$
C=C_{1}+\ldots+C_{k}=\sum_{x_{A}=1}^{k} C_{x_{A}}
$$

Additionally to the above, let us define:

$L=$ Occurrence of specified top event (e.g. core damage) by combination of failures (including the initiator) which does not include considered failure mode.

It can, then, be written:

$$
B=L+C A
$$

Having in mind the expression (5-1), the term $L$ would represent the logical sum of the minimal combinations which do not include failure mode $A$. Here, it needs to be pointed that the above expression for the top event $B$ assumes that top event (such as reactor core damage) can be logically presented in terms of failures, without the need for including the events representing successful responses in the logic expression. Such a presentation is used, in most of the cases, in the PRA models developed by the "FT linking" approach. (There is another PRA modeling approach, referred to as "ET linking," which is not discussed in this monograph). Logic expression for the top event is reduced to the form of logic sum of minimal cutsets (MCS) which represent minimal combinations of failures. "Success events", although taken into account (as negations of failures) for the proper MCS generation, are not explicitly shown in the final top event expression. In some cases (and depending on the PRA tool 
used) it may be possible to account for the quantitative impact of "success events" on the nominal top event probability. However, it would still not be possible (without overpassing some major issues) to incorporate this impact into calculation of risk importance measures.

\subsection{Theoretical relation between RAW and RRW}

We will start by pointing out that, theoretically (regardless of PRA modeling), the importance measures RAW and RRW for a single failure mode (represented by single basic event) are related to each other, with probability of considered failure mode as a parameter. If one of them is known, then the other is determined.

Based on their definitions in Section 4.2, the RAW importance measure $\left(I_{R A W}\right)$ and the RRW importance measure $\left(I_{R R W}\right)$ can, most generally, be defined as:

$$
I_{R A W}=\frac{P(B \mid A)}{P(B)}
$$

(i.e. the ratio of the conditional probability of top event under the assumption that considered component would always fail when challenged and the base case top event probability)

$$
I_{R R W}=\frac{P(B)}{P(B \mid \bar{A})}
$$

(i.e. ratio of the base case top event probability and the conditional probability of top event under the assumption that considered component would never fail when challenged).

To see the mentioned point, one can simply start from the above definition of RAW and expand it to:

$$
I_{R A W}=\frac{P(B \mid A)}{P(B)}=\frac{P(B A)}{P(B) P(A)}
$$

By considering $B=B(A+\bar{A})=B A+B \bar{A}$ and, consequentially, $P(B)=P(B A)+P(B \bar{A})$, the numerator can be rewritten as:

$$
P(B A)=P(B)-P(B \bar{A})
$$


The second term in (5-7) can be expanded to:

$$
P(B \bar{A})=P(B \mid \bar{A}) P(\bar{A})=\frac{P(B)}{I_{R R W}}[1-P(A)]
$$

By substituting (5-7) and (5-8) into (5-6) and rearranging, one obtains:

$$
I_{R R W}=\frac{1-P(A)}{1-P(A) I_{R A W}}
$$

From (5-9) it becomes obvious that $I_{R A W}=1$ implies $I_{R R W}=1$. In other words: statement $I_{R A W}=1$ a priori implies that top event is insensitive to the status of $A$ which further implies that $I_{R R W}$ can only be equal to 1 .

The above relation is demonstrated in Example B1 (Appendix B) by calculations based on the simplified RiskSpectrum ${ }^{\circledR}$ PRA model described in B.1.

For the end, it is pointed out that presented theoretical relation relies on the postulation that quantitative risk is represented by the probability of a top event, $P(B)$. The same kind of relation has been established and discussed, among others, in the references [28] and [32] and interested readers are invited to check those for additional insights.

\subsection{FC for a single basic event}

We start our considerations with fractional contribution (which is also called Fussel-Vesely importance). Its definition in NUREG/CR-3385 (provided here in Section 4.2) actually indicates that it can be calculated by a PRA model in two (complementary) ways:

1. Based on the difference between nominal risk and reduced risk, or

2. Based on direct quantification of all sequences involving considered failure event (i.e. those sequences which make the above difference).

Sometimes, these two complementary ways are used to make a distinction between FC and F-V (as an example, see Appendix A). However, in order to focus the discussions, the FC as a measure will have, in this monograph, the same meaning as F-V. It is noted, without further discussion, that in any PRA model which is coherent and has the failure probabilities within a range of current data bases (e.g. NUREG/CR-6928, 
[33]) the two ways of calculation should provide the results which would be, for all practical purposes, the same. To start the discussions in this monograph, we have chosen to formally define the FC in a manner which corresponds to the case 2 above. One of the reasons is that we want to establish the component-level FC (starting from basic events) and this way of expressing the FC makes it more convenient and transparent.

We will continue the discussions of risk importance measures for a single basic event in the following way:

+ First, we define, in this section, the FC by means of above introduced terms $A, B$ and $C$, and then

- We show, in the next three sections, that once FC is known, the other three considered importance measures can, under certain assumptions, be calculated from it.

It is noted that the discussion could start with RAW or with RRW, as well. Starting with FC is just a matter of convenience.

Considering the definitions of the terms $A, B$ and $C$ in the preceding section, the FC, as an importance measure, can now be, most generally, written as:

$$
I_{f_{c}}=\frac{P(C A B)}{P(B)}=\frac{P(C A)}{P(B)}
$$

The term in the numerator represents absolute contribution to the overall probability of top event. It is the probability of occurrence of any scenario leading to the challenge of considered component with regards to the failure mode represented by the basic event $A$, followed by the occurrence of this failure mode and, hence, of the top event (or, in other words: the probability that occurrence of top event involved failure of considered component). Note that, considering the expression for $B$ given by $(5-3)$ above: $P(C A B)=P(C A)$. This term will be, for convenience, designated as $I_{F C}$ and referred to as "absolute contribution":

$$
I_{F C}=P(C A)
$$

Since the basic events in the PRA fault trees are defined in a way to be independent, the above expression can further be developed to: 


$$
I_{F C}=P(C) P(A)
$$

Therefore:

$$
I_{f_{c}}=\frac{I_{F C}}{P(B)}=\frac{P(C) P(A)}{P(B)}
$$

As already pointed, knowing the FC makes it possible to directly calculate other three discussed importance measures. This is shown in corresponding three sections below. Some calculations involve approximations which are discussed and explored in examples based on the simplified PRA model. These approximations are considered to have negligible impacts on applications of practical importance.

\subsection{RAW for a single basic event}

RAW was already defined by the expression (5-4). Now it will be related to the FC, for which purpose certain approximations will be made, as mentioned above.

From (5-3), it can be written:

$$
B A=L A+C A
$$

Assuming that "rare event approximation" can be used for the terms $L A$ and $C A$ :

$$
P(B A) \approx P(L A)+P(C A)=P(L A)+I_{F C}
$$

It should be noted that the above approximation implies the following assumption:

$$
P(L A C)<<P(L A)+P(C A)
$$

This would be true as long as failures involved in minimal combinations in expressions (5-1) and (5-2) are low probability events. Example B2 (Appendix B) shows that this approaximation has no relevant impact on the exercises done with the simplified PRA model described in B.1.

The initial assumption was that the top event can be expressed in the form of a logical sum of minimal combination of failures (i.e. (5-1)). Under this logical sum, the term $L$ represents the sub-sum of combinations of failures which do not involve failure mode $A$. Therefore, it 
is reasonable to assume that the terms $L$ and $A$ are independent. (The term $L$ involves basic events which do not include basic event $A$. In a PRA, any reasonable effort is taken to make all the basic events involved in minimal cutsets mutually independent. This is necessary because the top event probability calculation in PRA models relies on the formulas which assume independency of basic events.) Thus:

$$
P(L A)=P(L) P(A)
$$

Considering (5-3), the term $P(L)$ can, using the "rare event approximation", further be written as:

$$
P(L) \approx P(B)-P(C A)=P(B)-I_{F C}
$$

It is noted that the approximation implies:

$$
P(B)-P(C A)>P(L A C)
$$

Example B3 (Appendix B) shows that this approaximation has no relevant impact on the exercises done with simplified PRA model described in B.1. The above enables (5-14) to be developed into:

$$
P(B A)=\left[P(B)-I_{F C}\right] P(A)+I_{F C}=P(B) P(A)+I_{F C}[1-P(A)]
$$

The RAW, defined by (5-4), can now be rewritten as:

$$
I_{R A W}=\frac{P(B \mid A)}{P(B)}=\frac{P(B A)}{P(A) P(B)}=1+\frac{I_{F C}}{P(B)}\left(\frac{1}{P(A)}-1\right)
$$

or, finally:

$$
I_{R A W}=1+I_{f_{c}}\left(\frac{1}{P(A)}-1\right)
$$

Therefore, if FC for particular basic event is known, its RAW can be directly calculated by the above expression. Such calculation is demonstrated in Example B4 (Appendix B) where calculated RAW values are compared against those reported by the PRA model. Some additional aspects in relating RAW to FC are discussed in a number of references, including [26], [28], [29], [32] and [34]. Interested readers are invited to check the references such as those for further insights. 


\subsection{RRW for a single basic event}

RRW, already defined by the expression (5-5), is also, related to FC. This can, simply, be shown by taking the expression (5-16) for RAW and considering the theoretical relation between RAW and RRW, given by $(5-9)$.

It is easy to show:

$$
I_{R R W}=\frac{P(B)}{P(B)-I_{F C}} \quad \text { or } \quad I_{R R W}=\frac{1}{1-I_{f_{c}}}
$$

This relation was already implied by the definitions of RRW and FC from NUREG/CR-3385 presented in Section 4.2. However, here it is confirmed for the definitions of RAW, RRW and FC made explicitly via the terms $A, B$ and $C$ from a PRA model.

If FC for particular basic event is known, then its RRW can be calculated by the above expression. Such calculation is demonstrated in Example B4 in Appendix B where calculated values are compared against reported values. Considering that $0<I_{f_{c}}<1$, it can be seen that RRW and FC would result with same ranking of basic events.

\subsection{Reliability importance for a single basic event}

Based on its definition in 4.2, the reliability importance can be formally written as:

$$
I_{\text {rel }}=\left.\frac{\partial P(B)}{\partial P(A)}\right|_{P(A)=Q_{A}}
$$

where $Q_{A}$ represents the "nominal" probability of $A$. The following can be considered:

$$
P(B)=P(B A)+P(B \bar{A})=P(A) P(B \mid A)+[1-P(A)] P(B \mid \bar{A}),
$$

The argument of the term $P(B \mid A)$, which essentially means $P(B \mid A=1)$, is free of " $A$ ". The same applies to the argument of the term $P(B \mid \bar{A})=P(B \mid \bar{A}=1)=P(B \mid A=0)$. Therefore, these two terms are not dependent on $P(A)$ and can, in the above expression, be considered as constants. 
40 Risk Importance Measures in the Design and Operation of NPPs

Hence:

$$
I_{\text {rel }}=\frac{\partial P(B)}{\partial P(A)}=P(B \mid A)-P(B \mid \bar{A})
$$

(Note that this corresponds to the definition of "Birnbaum importance" mentioned in Section 4.2.) It is worth noting that (5-20) was obtained theoretically, i.e. without any approximation.

By considering the definitions (5-4) and (5-5) this becomes:

$$
I_{\text {rel }}=P(B)\left(I_{R A W}-\frac{1}{I_{R R W}}\right)
$$

When above established relations for RAW and RRW, (5-18) and (5-17), are substituted into (5-21), the reliability importance is expressed via FC as:

$$
I_{r e l}=P(B) \frac{I_{f c}}{P(A)} \quad \text { or } \quad I_{r e l}=\frac{I_{F C}}{P(A)}
$$

The above formula can, also, be reached in a less formal way by considering:

$$
I_{\mathrm{rel}} \approx \frac{\Delta P(B)}{\Delta P(A)} \approx \frac{Q_{B, 2}-Q_{B, 1}}{Q_{A, 2}-Q_{A, 1}} .
$$

If the probabilities in the numerator are taken at $Q_{B, 1}=P(B)$ and $Q_{B, 2}=P(B \mid \overline{\mathrm{A}})$ then the probabilities in the denominator need to be taken at $Q_{A, 1}=P(A)$ and $Q_{A, 2}=0$. Hence:

$$
I_{\text {rel }} \approx \frac{P(B)-P(B \mid \bar{A})}{P(A)}
$$

By introducing $A=0$ ("false") into (5-3) one obtains $P(B \mid \bar{A})=P(L)$ (with due consideration to the earlier discussed assumption that $L$ contains only failures, without "success events"). By further considering (5-16), one finally obtains: 


$$
I_{\text {rel }} \approx \frac{P(B)-P(L)}{P(A)} \approx \frac{I_{F C}}{P(A)}=P(B) \frac{I_{f_{c}}}{P(A)}
$$

Thus, if FC is known, then reliability importance can be calculated by the above formula. Such calculation is demonstrated in Example B4 in Appendix B. 


\section{Risk importance of a component represented by multiple basic events, none involved in a CCF Group (FT module)}

\subsection{Introduction}

Many times, failure of a component is in fault trees represented by some kind of a logic structure. In other words, it is provided as some kind of a logic function defined over a set of basic events representing particular failure modes:

$$
A^{*}=a_{A}\left(A_{X}, A_{Y}, \ldots\right)
$$

In the above expression the asterisk $\left(^{*}\right)$ denotes that failure is represented by a logic function of basic events rather than by a single basic event. The term $a_{A}(\cdot)$ represents a logic function for the failure $A$ while the terms $A_{X}, A_{Y}, \ldots$ are basic events corresponding to particular failure modes $X, Y, \ldots$ etc.

If the following two conditions are fulfilled, the logic function $a_{A}\left({ }_{*}\right)$ can be referred to as a logic module or a fault tree module: 1) logic function $a_{A}({ })$ is the same wherever the failure $A^{*}$ appears in the fault tree structure; 2) none of the basic events $A_{X}, A_{Y}, \ldots$, appears in the fault tree structure externally to the logic function $a_{A}\left({ }_{+}\right)$. (In other words, a fault tree module is independent of the rest of the fault tree structure.) A fault tree module is, basically, a sub-tree with top event representing the failure of the considered component. It is pointed out that particular FT module (in the above sense) cannot involve a basic event which is shared by another FT module. Hence, it cannot involve a CCF basic event. Therefore, the represented component cannot be involved in a CCF group. Example of a FT module for a standby pump's failure on demand is shown in Section 6.3.

Formulas for FC, RAW and RRW, respectively, which were derived in Section 5 for a single basic event $A$, can be applied for a fault tree module $A^{*}$, as well. This is simply because if the logic module $A^{*}$ is replaced by a new single basic event (with assigned probability calculated for the whole module, $P\left(A^{*}\right)$ ), it would not affect general logic of the fault tree as a whole, or the probability of its top event.

We will retain the definition of $B$ as the overall top event and introduce the terms $A^{*}, C^{*}$ and $L^{*}$ with meaning analogous to $A, C$ and $L$, 
respectively, only that they relate to a FT module, rather than to a single basic event. Similarly, the asterisk will denote that an importance measure relates to a FT module.

\subsection{FC, RAW, RRW and reliability importance for component-level FT module}

Because the component failure basic events appearing in fault tree structure in a PRA are claimed to be independent, the above terms $C^{*}$ and $A^{*}$ are considered to be independent. (Note that, by the above definition of a FT module, the term $\mathrm{C}^{*}$ does not include any basic event from $\left.A^{*}\right)$. Hence, analogously to (5-11), the absolute contribution for a FT module can be written as:

$$
I_{F C}^{*}=P\left(C^{*} A^{*}\right)=P\left(C^{*}\right) P\left(A^{*}\right)
$$

The FC for a FT module is then defined analogously to (5-12):

$$
I_{f c}^{*}=\frac{I_{F C}^{*}}{P(B)}
$$

Once the FC for a considered FT module is known, the RAW can be calculated by a formula analogous to (5-17):

$$
I_{R A W}^{*}=1+\frac{I_{F C}^{*}}{P(B)}\left(\frac{1}{P\left(A^{*}\right)}-1\right) \text { or } I_{R A W}^{*}=1+I_{f c}^{*}\left(\frac{1}{P\left(A^{*}\right)}-1\right)
$$

Likewise, the RRW can be calculated analogously to (5-18) as:

$$
I_{R R W}^{*}=\frac{P(B)}{P(B)-I_{F C}^{*}} \quad \text { or } \quad I_{R R W}^{*}=\frac{1}{1-I_{f_{c}}^{*}}
$$

Finally, the corresponding reliability importance can be calculated analogously to (5-22) as:

$$
I_{r e l}^{*}=\frac{I_{F C}^{*}}{P\left(A^{*}\right)} \text { or } I_{r e l}^{*}=P(B) \frac{I_{f_{c}}^{*}}{P\left(A^{*}\right)}
$$

We provide a simple example with calculations of all four importance measures for a FT module in the next section. 


\subsection{Exampleः importance measures for a FT module for failure of a standby pump}

Many times, the FT module is simply an "OR" gate with its inputs being basic events representing different failure modes, i.e. the logic function from $(6-1)$ is given in the form:

$$
A^{*}=A_{X}+A_{Y}+\ldots
$$

An example of such a FT module, representing failures or unavailability of Positive Displacement Pump (PDP), is shown in Figure 6-1. Logic function for this simple FT module can be written as:

$$
A^{*}=A_{F S}+A_{F R}+A_{T M}
$$

where basic events $A_{F S}, A_{F R}$ and $A_{T M}$ relate to pump's failure to start, failure to run and unavailability (at the time of demand) due to test or maintenance (TM), respectively. (For simplicity, shorter notations are used than those for the basic events shown in the figure.)

The FT module from Figure 6-1 will be used to demonstrate calculation of component-level importance measures according to Section 6.2 above. It is noted that the Positive Displacement Pump (PDP) of Chemical and Volume Control System (CVCS) from the referential PRA model was selected for this example because it is not involved in any CCF group. It is also noted that TM unavailability is grouped with component's failure modes only for the sake of example for calculation of module-level importance measures. Such a grouping of TM unavailability with failures may or may not be appropriate, depending on the specific application.

First, the component-level failure probability $P\left(A^{*}\right)$ is needed for the logic function expressed by (6-8). It can be calculated as a probability of logic sum (by using the inclusion-exclusion principle). However, considering the range of the probabilities used in the PRAs for the failure modes of concern, the component level probability can be simplified to:

$$
P\left(A^{*}\right) \approx P\left(A_{F S}\right)+P\left(A_{F R}\right)+P\left(A_{T M}\right)
$$

(See, also, the discussion in Section 9.1.) Any of the three probabilities on the right hand side can be directly obtained from the PRA model. 


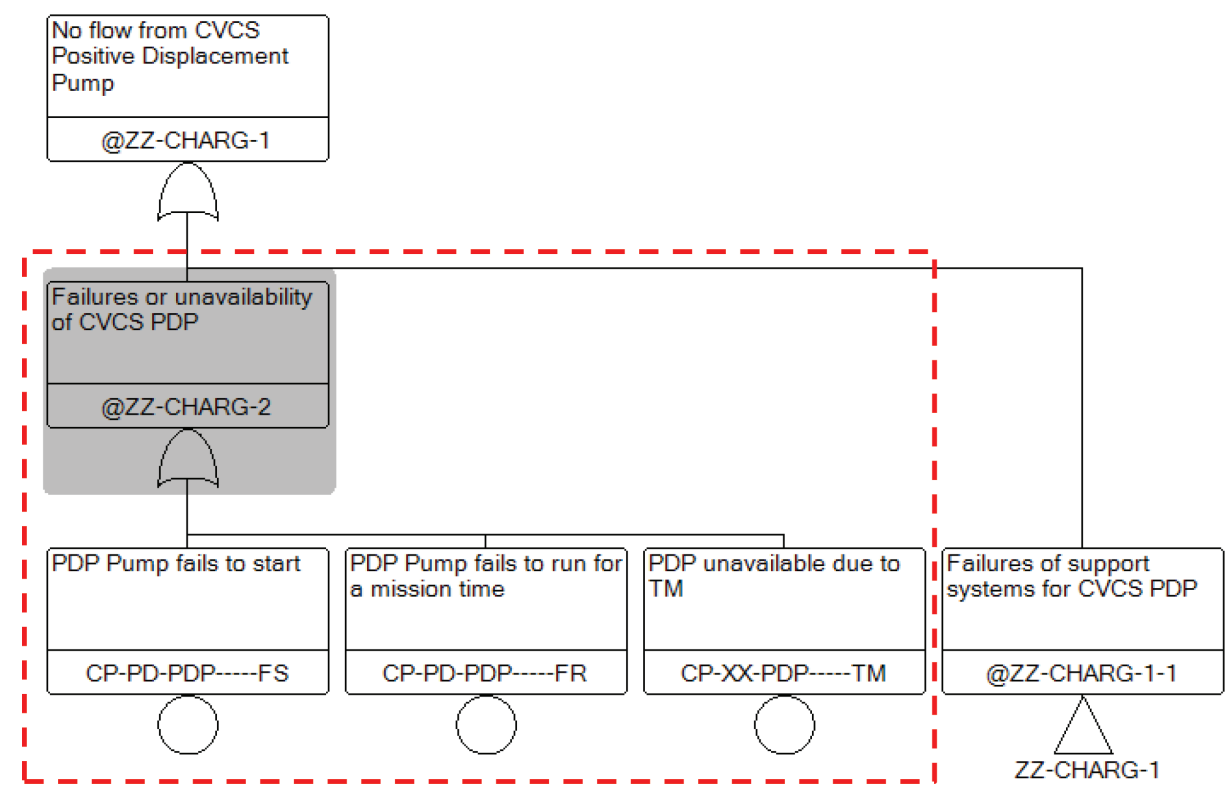

Figure 6-1 Example FT module (CVCS PDP).

Calculation of component-level importance measures starts with FC, which is then used to obtain other measures.

6.3.1 FC

With $P\left(A^{*}\right)$ known, the term $P\left(C^{*}\right)$ remains to be determined if the component-level FC is to be calculated. (See (6-2).) For the considered case this is straightforward: since the basic events appear under the "OR" gate, they will always be "challenged" by the same scenarios (representing an initiator and a combination of failures). The term " $C$ " is same for any of them and this same term represents the component-level term $C^{*}, i_{+} e_{*} C_{F S}=C_{F R}=C_{T M}=C^{*}$ and, accordingly:

$$
P\left(C_{F S}\right)=P\left(C_{F R}\right)=P\left(C_{T M}\right)=P\left(C^{*}\right)
$$

Any $P\left(C_{X}\right)$, index " $X$ " referring to " $F S$ ", " $F R$ " or "TM", can, according to $(5-11)$, be calculated as: $P\left(C_{X}\right)=\frac{I_{F C, X}}{P\left(A_{X}\right)}$. Therefore, considering $(6-2)$ and (6-3), the component-level FC is: 


$$
I_{f_{c}}^{*}=I_{f c}, X \frac{P\left(A^{*}\right)}{P\left(A_{X}\right)}
$$

The component-level FC can also be directly expressed by considering:

$$
\begin{aligned}
I_{F C}^{*} & =P\left(C^{*}\right) P\left(A^{*}\right) \approx P\left(C^{*}\right)\left[P\left(A_{F S}\right)+P\left(A_{F R}\right)+P\left(A_{T M}\right)\right] \\
& =I_{F C, F S}+I_{F C, F R}+I_{F C, T M}
\end{aligned}
$$

and, hence:

$$
I_{f c}^{*}=I_{f c, F S}+I_{f c, F R}+I_{f c, T M}
$$

Therefore, the component-level FC for the considered case, in which all failure modes are logically "OR-ed" together, can be obtained as a sum of corresponding FC values. The calculation of FC for this case is demonstrated by Example B5 in Appendix B.

6.3.2 RAW, RRW and reliability importance

With $I_{f_{c}}^{*}$ calculated above, component-level RAW, RRW and reliability importance can be calculated by formulas (6-4), (6-5) and (6-6), respectively. These calculations are also demonstrated in Example B5, Appendix B. 


\section{Risk importance of component represented by multiple different FT modules or basic events in different accident scenarios, none involved in a CCF Group}

\subsection{Introduction}

In this section we continue consideration of component-level importance measures for a component which is not involved in any CCF group. In Section 6 the component of concern was, wherever it appears in the PRA fault tree structure, always presented by the same FT module (sub-tree). For this reason, the logic expression for the top event could have been, with respect to the considered component, written as:

$$
B=L^{*}+C^{*} A^{*}
$$

(This is the expression (5-3) rewritten for a FT module, instead of a single basic event.) However, many times a particular component in a PRA model may be represented by different FT modules (sub-trees) or different single basic events for the same failure mode under different conditions imposed by different accident sequences. The examples may include:

- Higher likelihood of particular failure mode due to harsh ambient conditions created by particular initiators (e.g. high energy line break [HELB]) as compared to, e.g., general transient;

+ Different mission times applicable to different accident sequences (e.g. mission time for emergency feedwater turbine driven pump [EFW TDP] in the station blackout (SBO) sequence may be limited by the capacity of batteries (availability of instrumentation and control power), while in the general transient there may be no such limit);

+ Modeling of seismically induced failures of SSCs in a seismic PRA. (Different seismically induced failure probabilities apply when quantifying risk from different intervals of seismic intensity.)

In the case that different component-level FT modules apply in different event sequences the above expression (7-1) is to be rewritten as:

$$
B=L^{*}+\sum_{i=1}^{n} C_{i}^{*} A_{i}^{*}
$$


where $A_{i}^{*}$ refers to a specific FT module (or basic event) and $C_{i}^{*}$ to its corresponding $C$ term as defined in Sections 6 and 5. It should be noted that for every pair $i$ and $j(i \neq j)$ both apply: $A_{i}^{*} \neq A_{j}^{*}$ and $C_{i}^{*} \neq C_{j}^{*}$ It is important to stress that none of the FT modules $A_{i}^{*}$ involves a basic event which would represent a CCF shared by other components, as we are still considering a component which is not involved in any CCF group.

In order to be able to obtain the component-level expressions for the importance measures for this case, we will introduce additional term:

$$
\begin{aligned}
X_{i}= & \text { Challenge to the considered component comes from the fail- } \\
& \text { ure mode(s) represented by the FT module (or basic event) } A_{i}^{*}, \\
& \text { given the component has been challenged, } i=1, \ldots, n .
\end{aligned}
$$

In order to simplify the evaluation it will be assumed that any $X_{i}$ and $X_{j}, i \neq j$, are mutually exclusive (i.e. $\sum_{i=1}^{n} X_{i}=1$, where sum refers to the union and " 1 " refers to the complete set of hypotheses). This assumption is a simplification. However, it can be supported by the point that different FT modules (or basic events representing failure modes under different conditions) of the considered component usually appear under different initiators (ETs) or different sequences under the same ET. Initiator categories in the PRA are usually defined in a way to be mutually exclusive (e.g. if LOCA event is "large", then it is not "medium"; if reactor trip is due to loss of connection to grid then it is "loss of offsite power" initiator, otherwise it is one of "transients", etc.). Different sequences within the same ET are mutually exclusive by definition (because each pair of sequences involves at least one function or system with opposite status). (It is noted here that minimal cutsets may not explicitly show the "success events" and for this reason some cases of mutual exclusivity may not be explicit. However, this is one of the PRA issues with broader implications and their evaluation is far beyond the scope of this discussion.)

The above assumption implies that challenges $C_{i}^{*}(i=1, \ldots, n)$ are mutually exclusive, which is explained by the same rationale (i.e. mutual exclusivity of event sequences in PRA). It enables introducing general 
term $C^{*}$ which represents occurrence of any scenario or combination of events (initiator followed by failures or errors) which requires successful operation of considered component in order to prevent the top event (e.g. reactor core damage) from occurring, and which is defined as:

$$
C^{*}=C^{*} \sum_{i=1}^{n} X_{i}=\sum_{i=1}^{n} X_{i} C^{*}=\sum_{i=1}^{n} C_{i}^{*} \text { where } C_{i}^{*}=X_{i} C^{*}, i=1, \ldots, n,
$$

It is now, also, possible to introduce effective general failure of considered component upon challenge. The sum in (7-2) can now be rewritten as:

$$
\sum_{i=1}^{n} C_{i}^{*} A_{i}^{*}=\sum_{i=1}^{n}\left(C^{*} X_{i}\right) A_{i}^{*}=C^{*} \sum_{i=1}^{n} X_{i} A_{i}^{*}=C^{*} A^{*}
$$

where

$$
A^{*}=\sum_{i=1}^{n} X_{i} A_{i}^{*}
$$

represents the mentioned effective failure of considered component. At the same time, this brings (7-2) back to the general form expressed by (7-1), meaning that the problem of calculating component-level importance measures is reduced to the one already discussed in Section 6. The same equations can be used for FC, RAW, RRW and reliability importance. However, to calculate the "effective" total failure probability $P\left(A^{*}\right)$, the terms $P\left(X_{i}\right)$ (conditional probabilities) will have to be known for all particular FT-modules $A_{i}^{*}(i=1, \ldots, n)$. This is further discussed in the sections which follow. Sections 7.2 through 7.4 establish the expressions for all four importance measures for this case. Section 7.5 provides an example based on the simplified PRA model described in B.1, Appendix B.

\subsection{FC}

With above introduced terms considered, deriving the expression for FC measure for a component represented by a group of FT modules $A_{i}^{*}$, $i=1, \ldots, n$, is straightforward.

Starting from the most general expressions for absolute contribution, $(5-10)$ and $(6-2)$, and considering mutual exclusivity of terms $\left(C_{i}^{*} A_{i}^{*}\right)$, discussed above: 


$$
I_{F C}^{*}=P\left(C^{*} A^{*}\right)=P\left(\sum_{i=1}^{n} C_{i}^{*} A_{i}^{*}\right)=\sum_{i=1}^{n} P\left(C_{i}^{*} A_{i}^{*}\right)=\sum_{i=1}^{n} I_{F C, i}^{*}
$$

where the term $I_{F C, i}^{*}(i=1, \ldots, n)$ represents the absolute contribution of the FT module $A_{i}^{*}$.

Therefore, the absolute and fractional contributions of a component represented by $n$ different FT modules (basic events) $A_{i}^{*}$ in the different sequences in PRA model can be written as:

$$
I_{F C}^{*}=\sum_{i=1}^{n} I_{F C, i}^{*} \quad \text { and } \quad I_{f c}^{*}=\sum_{i=1}^{n} I_{f c, i}^{*}
$$

With component-level FC known, it will be possible to calculate other importance measures, as it was in the previous cases.

\subsection{RAW}

The formula (6-4), which is generalized form of (5-17), can be used to calculate the RAW for a component represented by multiple FT modules. However, the total effective failure probability $P\left(A^{*}\right)$ needs to be known. From $(7-5)$ and the assumption that the terms $X_{i}(i=1, \ldots, n)$ are mutually exclusive:

$$
P\left(A^{*}\right)=\sum_{i=1}^{n} P\left(X_{i} A_{i}^{*}\right)
$$

Any term $X_{i}$ is related to challenges to the component (specific challenge $C_{i}^{*}$ and general challenge $C^{*}$ ) and not to the performance of the component. Therefore, it can be claimed independent from $A_{i}^{*}$ (on the same basis on which $C_{i}^{*}$ was earlier claimed independent from $\left.A_{i}^{*}\right)$.

Therefore:

$$
P\left(A^{*}\right)=\sum_{i=1}^{n} P\left(X_{i}\right) P\left(A_{i}^{*}\right)
$$

Thus, set of the conditional probabilities $P\left(X_{i}\right)(i=1, \ldots, n)$ needs to be known in order to be able to calculate $P\left(A^{*}\right)$. Particular term $P\left(X_{i}\right)$ can, according to the definition of $X_{i}$, be written as: 


$$
P\left(X_{i}\right)=P\left(C_{i}^{*} \mid C^{*}\right)=\frac{P\left(C_{i}^{*} C^{*}\right)}{P\left(C^{*}\right)}=\frac{P\left(C_{i}^{*}\right)}{P\left(C^{*}\right)}=\frac{P\left(C_{i}^{*}\right)}{\sum_{i=1}^{n} P\left(C_{i}^{*}\right)}
$$

In the above derivation, it is considered that $C_{i}^{*}$ implies $C^{*}$ and that terms $C_{i}^{*}(i=1, \ldots, n)$ are assumed to be mutually exclusive. Considering that $P\left(C_{i}^{*} A_{i}^{*}\right)=I_{F C, i}$ (absolute contribution of FT-module $\left.A_{i}^{*},(7-6)\right)$ and that $C_{i}^{*}$ and $A_{i}^{*}$ are claimed independent $\left(P\left(C_{i}^{*} A_{i}^{*}\right)=P\left(C_{i}^{*}\right) P\left(A_{i}^{*}\right)\right.$, $(6-2))$ :

$$
P\left(C_{i}^{*}\right)=\frac{I_{F C, i}^{*}}{P\left(A_{i}^{*}\right)}
$$

With this, the conditional probability $P\left(X_{i}\right)(i=1, \ldots, n)$ can, finally, be expressed as:

$$
P\left(X_{i}\right)=\frac{\frac{I_{F C, i}^{*}}{P\left(A_{i}^{*}\right)}}{\sum_{i=1}^{n} \frac{I_{F C, i}^{*}}{P\left(A_{i}^{*}\right)}}=\frac{\frac{I_{f c, i}^{*}}{P\left(A_{i}^{*}\right)}}{\sum_{i=1}^{n} \frac{I_{f c, i}^{*}}{P\left(A_{i}^{*}\right)}}
$$

and total effective failure probability $P\left(A^{*}\right)$ becomes:

$$
P\left(A^{*}\right)=\sum_{i=1}^{n} \frac{I_{F C, i}^{*}}{\sum_{i=1}^{n} \frac{I_{F C, i}^{*}}{P\left(A_{i}^{*}\right)}}=\frac{I_{F C}^{*}}{\sum_{i=1}^{n} \frac{I_{F C, i}^{*}}{P\left(A_{i}^{*}\right)}}=\frac{I_{f_{c}}^{*}}{\sum_{i=1}^{n} \frac{I_{f c, i}^{*}}{P\left(A_{i}^{*}\right)}}
$$

Thus, RAW for a component with multiple FT modules can be expanded into:

$$
I_{R A W}^{*}=1+\frac{I_{F C}^{*}}{P(B)}\left(\frac{1}{P\left(A^{*}\right)}-1\right)=1+\frac{1}{P(B)}\left(\sum_{i=1}^{n} \frac{I_{F C, i}^{*}}{P\left(A_{i}^{*}\right)}-I_{F C}^{*}\right)
$$


or:

$$
I_{R A W}^{*}=1+I_{f c}^{*}\left(\frac{1}{P\left(A^{*}\right)}-1\right)=1+\sum_{i=1}^{n} \frac{I_{f c, i}^{*}}{P\left(A_{i}^{*}\right)}-I_{f_{c}}^{*}
$$

It is noted that, because $P\left(A_{i}^{*}\right)<1(i=1, \ldots, n)$, it will always be $\sum_{i=1}^{n} \frac{I_{F C, i}^{*}}{P\left(A_{i}^{*}\right)}>I_{F C}^{*}\left(\right.$ and, consequentially, $\left.I_{R A W}>1\right)$.

\subsection{RRW and reliability importance}

The RRW and reliability importance for a component with multiple FT modules can be directly calculated by (6-5) and (6-6), respectively, by using the component-level FC and above derived total effective failure probability $P\left(A^{*}\right)$.

\subsection{Exampleः standby pump with two different operating failure rates}

It is very important to be able to properly define different FT-modules $A_{i}^{*}$ and accompanying challenges $C_{i}^{*}$, because the results of componentlevel importance calculations depend on this. Proper identification of FT-modules may call for "detailed" knowledge of the PRA model. We try to illustrate these points on the following example.

The example continues with CVCS Positive Displacement Pump from 6.3. For the sake of illustration, it will be assumed that pump has considerably higher operating failure rate under the conditions created by the initiators "Total Loss of Component Cooling Water (CCW)" and "Total Loss of Essential Service Water (ESW)" than its nominal failure rate. (For example, this may be due to loss of air conditioning unit in the pump's room, which is cooled by the CCW.) For this reason, pump's failure to run for the mission time needs for these two initiators to be modeled by a basic event different from the one which represents the nominal failure rate (Figure 6-1). Therefore, in this case we have the CVCS represented by two different FT modules which apply in different event sequences:

$$
A_{1}^{*}=A_{F S}+A_{F R}+A_{T M} ; A_{2}^{*}=A_{F S}+A_{F R, L}+A_{T M}
$$


In $(7-15)$ the term $A_{F R}$ represents "failure to run" basic event with nominal failure rate (same as in 6.3) while the term $A_{F R, L}$ represents "failure to run" basic event with higher failure rate which applies under the initiators "Total Loss of CCW" and "Total Loss of "ESW". The applicability of basic events and the two FT modules is presented by Figure 7-1, which, also, shows the applicable module-level "C terms".

It is noted here that in the above discussions under Section 7 no statement was made regarding the dependency or mutual exclusivity of the component-level failure modes represented by the FT modules $A_{i}^{*}, i=1, \ldots, n$. The only assumption which was made was regarding the mutual exclusivity of the challenges, i.e. terms $X_{i}\left(\right.$ and, hence, $\left.C_{i}^{*}\right)$. Thus, in this example we have two component-level failure modes $A_{1}^{*}$ and $A_{2}^{*}$ which are obviously not mutually exclusive. However, they are assumed to be challenged in a mutually exclusive way (Figure 7-1), which comes from the PRA assumption on exclusivity of initiator categories.

First, module-level failure probabilities are calculated for the two modules from (7-15) above, in the same manner as in (6-9):

$$
\begin{gathered}
P\left(A_{1}^{*}\right) \approx P\left(A_{F S}\right)+P\left(A_{F R}\right)+P\left(A_{T M}\right) \\
P\left(A_{2}^{*}\right) \approx P\left(A_{F S}\right)+P\left(A_{F R, L}\right)+P\left(A_{T M}\right)
\end{gathered}
$$

As before, the calculation of component-level importance measures starts with FC, which is then used to obtain other measures.

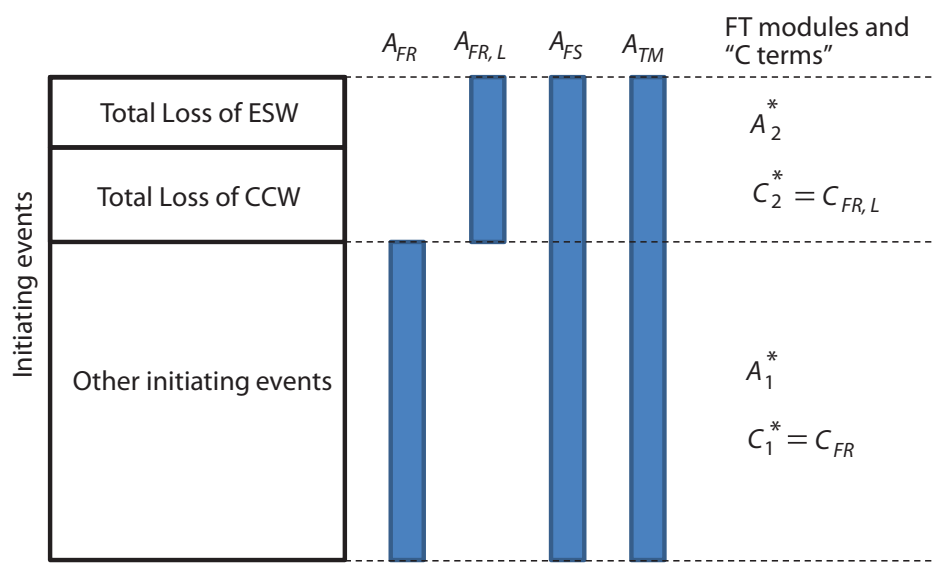

Figure 7-1 Applicability of basic events and the two FT modules (CVCS PDP example). 


\subsubsection{FC}

With module-level probabilities $P\left(A_{1}^{*}\right)$ and $P\left(A_{2}^{*}\right)$ known, the component-level absolute contribution can be calculated by $(7-6)$ if module-level challenges $C_{i}^{*}, i=1,2$, are known:

$$
\mathrm{I}_{\mathrm{FC}}^{*}=I_{F C, 1}^{*}+I_{F C, 2}^{*}=P\left(C_{1}^{*} A_{1}^{*}\right)+P\left(C_{2}^{*} A_{2}^{*}\right)
$$

From Figure 7-1 it can be seen that $C_{1}^{*}=C_{F R}$ (i.e. the "C-term" which corresponds to the basic event $A_{F R}$ ) and $C_{2}^{*}=C_{F R, L}$ (i.e. the "C-term" which corresponds to the basic event $\left.A_{F R, L}\right)$. Therefore:

$$
I_{F C}^{*}=P\left(C_{F R} A_{1}^{*}\right)+P\left(C_{F R, L} A_{2}^{*}\right)=P\left(C_{F R}\right) P\left(A_{1}^{*}\right)+P\left(C_{F R, L}\right) P\left(A_{2}^{*}\right)
$$

The terms $C$ and $A$ in the above expression are assumed to be independent based on the definitions of basic events in PRA as independent events. Expression (7-11) (which applies to FT module or to a single basic event) can now be used to calculate the terms $P\left(C_{F R}\right)$ and $P\left(C_{F R, L}\right)$ :

$$
P\left(C_{F R}\right)=\frac{I_{F C, F R}}{P\left(A_{F R}\right)} ; \quad P\left(C_{F R, L}\right)=\frac{I_{F C, F R, L}}{P\left(A_{F R, L}\right)}
$$

Therefore:

$$
\begin{aligned}
& I_{F C}^{*}=\frac{I_{F C, F R}}{P\left(A_{F R}\right)} P\left(A_{1}^{*}\right)+\frac{I_{F C, F R, L}}{P\left(A_{F R, L}\right)} P\left(A_{2}^{*}\right) ; \\
& I_{f c}^{*}=\frac{I_{f c, F R}}{P\left(A_{F R}\right)} P\left(A_{1}^{*}\right)+\frac{I_{f c, F R, L}}{P\left(A_{F R, L}\right)} P\left(A_{2}^{*}\right)
\end{aligned}
$$

In the above equations the terms $I_{f c}, F R$ and $I_{f c}, F R, L$ represent FCs of basic events $A_{F R}$ and $A_{F R, L}$ which can be directly obtained from the PRA results. Calculation of component-level FC is, for this case, demonstrated in Example B6 (Appendix B).

\subsubsection{RAW}

Starting from $(7-14)$ :

$$
I_{R A W}^{*}=1+\frac{I_{f c, 1}^{*}}{P\left(A_{1}^{*}\right)}+\frac{I_{f c, 2}^{*}}{P\left(A_{2}^{*}\right)}-I_{f \mathcal{c}}^{*}
$$


All terms can be directly "read" from (7-19). Thus:

$$
I_{R A W}^{*}=1+\frac{I_{f c, F R}}{P\left(A_{F R}\right)}+\frac{I_{f c, F R, L}}{P\left(A_{F R, L}\right)}-I_{f c}^{*}
$$

Calculation of component-level RAW for this case is demonstrated in Example B6 in Appendix B.

7.5.3 RRW and reliability importance

The component-level RRW and reliability importance are calculated from $I_{f_{c}}^{*}$ according to $(6-5)$ and (6-6), respectively. This is also demonstrated in Example B6. 


\section{Risk importance of component represented by a single basic event (failure mode) involved in a CCF Group}

\subsection{Introduction}

Up to now we have considered importance measures for a component represented by a FT module (sub-tree) involving a group of basic events (Section 6) or, even, a component represented by multiple different FT modules which appear in mutually exclusive sequences of failures (Section 7). However, none of the underlying basic events was involved in any common cause failure (CCF) group of basic events. (In other words, none of those failures or failure modes was shared by another components.) We will now broaden the discussion to the componentlevel risk importance measures for the components which are involved in a CCF group (which is, many times, the case with most of components in a PRA model). We start, in this section, with discussing the impact of CCF on risk importance of component which is presented in the FT structure by a single basic event.

With respect to risk importance of the component (or particular failure mode) involved in a CCF group, three types of a failure or unavailability should be distinguished:

1. Failure or unavailability with CCF potential;

2. Unavailability with no impact on CCF (e.g. unavailability due to preventive maintenance);

3. Particular CCF event (i.e. failure due to a common cause of specified components).

For the first one it can be said that it is mostly related to design issues. For the remaining two it can be said that they are primarily related to operational issues. All three cases are further discussed in the separate subsections below.

All the calculations and use of the formulas are demonstrated in Example B7 in Appendix B. 


\subsection{Failure with CCF potential}

Basic characteristic of this type of failure is that the considered component has failed and this can have implications on the status of the redundant components which are members of the same "CCF group". Specifically, the status (failed or operable) of all other redundant components is defined by the conditional probabilities used to quantify the CCF model in the PRA. To take the simplest example of a beta-factor CCF model for two redundant components, with assumed beta-factor value of 0.1 : assuming that first component has failed, there is $10 \%$ probability that the second component is also failed (and remaining $90 \%$ probability that failure had affected only the first component).

Risk importance of this type of failure is usually considered in evaluations of design or its modifications. For example, answering a question such as "what is the worth or significance of adding another redundant component or train?" is related to the risk importance of this type of failure.

We will start the discussion by considering a single basic event $A$ which in the PRA model represents specific failure mode of certain component of concern. In the case that represented failure mode is not shared by any other component modelled, the basic event $A$ would have importance measures as presented in Section 5. However, if the component is included in a CCF group, then considered failure mode of this component can be shared by a number of combinations of other components. We will simplify the notation and will not go into the details on the CCF modeling and quantification as they are described in a number of references (for example, NUREG/CR-4780 [35], or NUREG/CR-5845 [36]).

If considered component is involved in a CCF group of size $m$, the number of combinations of failures in which considered component can be involved is:

$$
M=\sum_{j=0}^{m-1}\left(\begin{array}{c}
m-1 \\
j
\end{array}\right)=2^{m-1}
$$

For example, let us consider a CCF group consisting of $m=4$ components designated, for simplicity, as 1,2, 3 and 4, and let us focus on the component 1 . According to the above formula, this component can be involved in $2^{3}=8$ combinations of failures, and they are: $(1),(1,2)$, $(1,3),(1,4),(1,2,3),(1,3,4),(1,2,4)$ and $(1,2,3,4)$. 
For simplicity, we will refer to each of these combinations simply as "combination $k$ " and have in mind that $k$ goes from 1 to $2^{m-1}=M$.

The following set of $m$ conditional mutually exclusive events can now be defined:

$U_{k}$ Failure of considered component is involved in combination $k$, $k=1, \ldots, M\left(\right.$ where $\left.\sum_{k=1}^{M} U_{k}=1\right)$.

The failure of component $A$ (which was previously presented by a single basic event $A$ ) is now partitioned into the set of mutually exclusive failure events $V_{k}=U_{k} A, k=1, \ldots, M$;

$$
A=\sum_{k=1}^{M} V_{\mathrm{k}}=\sum_{k=1}^{M} U_{\mathrm{k}} A=A \sum_{k=1}^{M} U_{\mathrm{k}}=A
$$

(Mutual exclusivity of these basic events may be subject to discussion or include certain degree of controversy. However, the fact is that at two most widely used CCF quantification methods, Multiple Greek Letters and Alpha Factors, the formulas for the set of conditional probabilities assume mutual exclusivity. Refer, e.g., to [35] or [36].)

In expression (8-2) we purposefully omitted use of the asterisk $\left(^{*}\right)$ with $A$, even if $A$ is a kind of logic similar to "FT module" considered earlier. The reason is that in Sections 6 and 7 we have used the term "FT module" for a logic structure representing particular component, which is independent from the remaining FT structure in the PRA model. In some instances, as we have seen in Section 7, multiple "FT modules" representing the same component may be mutually dependent (involving same basic events). However, they were always independent from the rest of the FT structure. With logic expression (8-2) this is not the case, by definition. All except one of the basic events $V_{k}, k=1, \ldots, M$, are also involved in the logic structures representing other components in every "combination $k$ ".

The expressions for the four component-level risk importance measures are discussed in the Sections 8.2.1 through 8.2.4. Demonstrations are provided under Example B7 in Appendix B.

Calculation of importance measures for failure modes which are involved in CCF groups are discussed in a number of references, 
including [27], [28] and [29]. (It is noted that subject matter of [27] is not, strictly, calculation of importance measures but, rather, calculation of a conditional risk for precursor events. However, the discussion of CCF impact is relevant and relates to the importance measures.) The focus of discussions is on RAW which is, in many practical evaluations, the most relevant of the measures (or the most difficult to asses) when $\mathrm{CCF}$ is involved. Interested readers are invited to check those references for further insights.

\subsubsection{FC}

General expression for the top event (5-3) needs now to be rewritten as:

$$
B=L^{\prime}+\sum_{k=1}^{M} C_{k} V_{k}
$$

Terms $C_{k}, k=1, \ldots, M$, represent scenarios or combinations of events such that specific CCF combination $k$ (involving component $A$ ) is requested not to occur, in order to avoid the top event (e.g. reactor core damage). The term $L^{\prime}$ (which is not, generally, the same as $L$ in (5-3)) represents an occurrence of the top event such that it does not include any CCF combination $k$.

Note that top event minimal cutsets now show particular basic events $V_{k}, k=1, \ldots, M$ (and not failure event $A$ explicitly, anymore) and this is why transformation to (8-3) is important. However, considering that $V_{k}=U_{k} A, k=1, \ldots, M$, the expression (8-3) can be transformed to

$$
B=L^{\prime}+A \sum_{k=1}^{M} C_{k} U_{k}=L^{\prime}+C^{\prime} A
$$

i.e. to the same general form as (5-3). Based on this, the general expression for the absolute contribution from Section 5, (5-10), can be used, i.e.:

$$
I_{F C}=P\left(C^{\prime} A\right)
$$

This expression does not enable actual calculation of the FC since the term $A$ is not shown in minimal cutsets. For this reason, the term $I_{F C}$ needs to be expressed through the basic events $V_{k}, k=1, \ldots, M$, i.e.* 


$$
I_{F C}=P\left(C^{\prime} A\right)=P\left(\sum_{k=1}^{M} C_{k} V_{k}\right)
$$

Considering that the terms $C_{k} V_{k}, k=1, \ldots, M$ are mutually exclusive (because the CCF events $V_{k}, k=1, \ldots, M$ were defined as mutually exclusive), the above expression can, similarly as in Section 7.2, be rewritten as:

$$
I_{F C}=\sum_{k=1}^{M} P\left(C_{k} V_{k}\right)
$$

Thus, the absolute and fractional contribution for a single failure mode which is involved in a CCF group can be calculated as

$$
I_{F C}=\sum_{k=1}^{M} I_{F C, k} \quad \text { and } \quad I_{f c}=\sum_{k=1}^{M} I_{f c, k}
$$

where the terms $I_{F C, k}$ and $I_{f c, k}$ represent the contributions of the CCF basic events $V_{k}, k=1, \ldots, M$. These basic events are explicitly shown in minimal cutsets and their importance measures can, therefore, be taken directly from the PRA results. Thus, component-level FC can be calculated directly.

Once the FC is known, it will be possible to calculate other importance measures for the considered component (or particular failure mode).

\subsubsection{RAW}

By starting with most general expression for the RAW, i.e. (5-4), and then considering the rewritten form of logic expression for top event $\mathrm{B}$ given by (8-3) and (8-4) it is not difficult to show that the RAW can be expressed by the formula which corresponds to $(5-17)$ :

$I_{R A W}=1+\frac{I_{F C}}{P(B)}\left(\frac{1}{P(A)}-1\right) \quad$ or $\quad I_{R A W}=1+I_{f_{c}}\left(\frac{1}{P(A)}-1\right)$

Here, $I_{F C}$ and $I_{f_{c}}$ are total contributions calculated by (8-8), while $P(A)$ represents the total failure probability expressed, based on (8-2), as: 
64 Risk Importance Measures in the Design and Operation of NPPs

$$
P(A)=\sum_{k=1}^{M} P\left(V_{k}\right)
$$

In deriving the formula (8-9) the same kind of rationale is used as in Section 5.4, including the application of "rare event approximation".

It is important to recognize the following: by stating $A=1$ (i.e. component $A$ has failed) we are not setting to "true" all CCF basic events which involve $A$, i.e. $V_{k}, k=1, \ldots M$. Instead, we are modifying their probabilities to conditional values. This can be seen by considering $V_{k}=U_{k} A, k=1, \ldots M$. With setting $A=1$ ("true") $V_{k}$ becomes $U_{k}$ and, correspondingly, $P\left(V_{k}\right)=P\left(U_{k}\right)$. This is important for understanding how the component-level importance measure should be calculated by modifying and rerunning the PRA model, as demonstrated in Example B7, Appendix B.

Interested readers are, also, directed to earlier mentioned references such as [27], [28] and [29] for related discussions.

\subsubsection{RRW and reliability importance}

Theoretical relation between RAW and RRW discussed in 5.2 is valid regardless of whether a failure mode is involved in a CCF or not. Therefore, it can be used to directly calculate RRW from RAW (formula (5-9)), with $P(A)$ calculated by (8-10). Alternatively, it can be used to express RRW via FC in the formula corresponding to $(5-18)$ :

$$
I_{R R W}=\frac{P(B)}{P(B)-I_{F C}} \quad \text { or } \quad I_{R R W}=\frac{1}{1-I_{f_{c}}}
$$

Likewise, reliability importance can theoretically be expressed through RAW and (or) RRW by (5-21) regardless of involvement in CCF. Therefore, it can be calculated directly from RAW and RRW, or it can be expressed via FC by the formula corresponding to (5-22), i.e.:

$$
I_{\text {rel }}=P(B) \frac{I_{f_{c}}}{P(A)} \quad \text { or } \quad I_{r e l}=\frac{I_{F C}}{P(A)}
$$

(where $P(A)$ is calculated by $(8-10)$ ). 
However, there are potential issues with interpretation of reliability importance in the case of involvement in a CCF group. Those are discussed under Example B7 in Appendix B.

\subsection{Unavailability with no impact on CCF}

In this case the fact that considered component is not able to perform its function upon demand does not provide any additional information regarding possible status of the remaining components in the CCF group. A case like this is best represented by assuming that considered component, which was otherwise normally available and operable, is taken to out-of-service status for the purpose of preventive maintenance. This action does not imply anything regarding the possible status (i.e. operable or inoperable) of other components in the CCF group. Basically, it only means that if considered component would be needed during the time of this unavailability, it would not be there to operate.

This kind of unavailability (and its associated importance) is considered in PRA applications which support plant operation, such as configuration risk monitoring.

It implies that redundancy of the system of $m$ components has been reduced to the redundancy of $m-1$ components. In other words, the CCF group of size $m$ has been reduced to the CCF group of size $m-1$ formed by the remaining components. However, the failure causes which the remaining components share with considered component can still affect them, regardless of the fact that considered component is now outof-service. The simplest example to illustrate this is the one with two redundant components $S_{1}$ and $S_{2}$. Let us assume that they each have total failure probability $P\left(S_{1}\right)=P\left(S_{2}\right)=Q_{t}$. Let us further assume that conditional probability of failure of any of the two being due to a cause shared with the other one is $\beta$ (so-called beta-factor.) If the first component has been taken down for the preventive maintenance, failure probability for the second one would still remain $(1-\beta) Q_{t}+\beta Q_{t}=Q_{t}$, because this failure probability is not influenced by the fact that the first component is being subjected to preventive maintenance. To use the notation from the previous section, failure probability of the second component would be $Q_{t}=P\left(V_{1}\right)+P\left(V_{2}\right)$, where $P\left(V_{1}\right)=(1-\beta) Q_{t}$ and $P\left(V_{2}\right)=\beta Q_{t}$

This discussion can be applied to the general case of component $A$ involved in a CCF group of $m$ components, expressed by $(8-2)$. This 
expression is done in terms of $M=2^{m-1}$ mutually exclusive basic events $V_{k}, k=1, \ldots, M$ which represent common cause failures of all combinations of components involving the considered component. For convenience, we will specifically define $V_{1}$ as basic event which represents failure of $A$ not shared by any other component from the group. (Note that in terms of MGL quantification model, the probability of this basic event would be $P\left(V_{0}\right)=\left(1-\beta^{(m)}\right) Q_{t}$, with $\beta^{(m)}$ representing corresponding beta-factor for the CCF group of $m$ components and $Q_{t}=P(A)$.) Since the definition of this kind of unavailability includes that there is no impact on other CCF events, the importance measures can be taken from the basic event $V_{1}$. Thus, absolute and fractional contribution of considered component $A$ with respect to this kind of unavailability can be expressed as:

$$
I_{F C}=I_{F C, 1} \quad \text { and } \quad I_{f_{c}}=I_{f_{c}, 1}
$$

where the terms $I_{F C, 1}$ and $I_{f c, 1}$ represent absolute and fractional contribution values for the basic event $V_{1}$. They can be obtained directly from the PRA results. All other importance measures can then be calculated as for any other single basic event, as described in Section 5, or they can be taken directly from the reported importance measures for the basic event $V_{1}$. However, it needs to be noted that actual meaning (i.e. the meaning for any practical purpose) of measures other than RAW in this case is questionable. The meaning and usability of RAW is clear: it is the measure of how much risk would increase assuming that component is taken out-of-service for preventive maintenance (without any impact on other components). However, this is not so with FC or RRW because it is not clear whether setting $V_{1}=0$ ("false") without any impact on other $V_{k}$ basic events can reflect any real situation in the practice. The same question can be asked about changing the probability $P\left(V_{1}\right)$ without changing probabilities of other $V_{k}$ basic events.

Finally, let it also be mentioned that in the base case PRA unavailability due to preventive maintenance is usually represented by a separate basic event and this event can be used to calculate the fractional contribution of this kind of activities and benefit from reducing their scope, if necessary.

For these reasons, the demonstrations under Example B7 in Appendix B focuses only on RAW importance for this case. It is taken directly 
from the reported importance measures for the basic event $V_{1}$ and used only to put into perspective the RAW in the case of CCF potential, which was discussed in 8.2.2.

\subsection{Particular CCF event}

This third case simply means that considered component is failed and failure is shared by specific $j$ other components from the CCF group, where $j=1, \ldots, m-1$. (It is noted that $j=0$ is special case which was addressed in Section 8.3.)

The risk importance of this kind of failure may, sometimes, be considered in PRA applications such as assessing risk significance of observed operational events or issues. It is included here for completeness purposes.

The most important case, by all means, is common cause failure of all $m$ components in the group. This specific case is discussed here. Referring to earlier defined set of $M=2^{m-1}$ mutually exclusive basic events $V_{k}, k=1, \ldots, M$ with respect to common cause failures of component $A$ we will, for convenience, define basic event $V_{M}$ as, specifically, being the failure of component $A$ which is shared by all other components in the group. (For $m=2, P\left(V_{M}\right)=P\left(V_{2}\right)=\beta^{(2)} Q_{t}$; for $m=3$, $P\left(V_{M}\right)=P\left(V_{4}\right)=\beta^{(3)} \gamma^{(3)} Q_{t}$, etc. $)$

Without further discussion, we will here just use the importance measures of basic event $V_{M}$ as representatives for risk importance associated with CCF of all components in the group. Thus, absolute and fractional contribution will be expressed as:

$$
I_{F C}=I_{F C, M} \quad \text { and } \quad I_{f c}=I_{f c, M}
$$

where the terms $I_{F C, M}$ and $I_{f c, M}$ represent absolute and fractional contribution values for the basic event $V_{M}$. They can be obtained directly from the PRA results.

Other importance measures for the CCF of all $m$ components can then be calculated as for any other single basic event, as described in Section 5 or they can be taken directly from the reported importance measures for the basic event $V_{M}$. However, similar question can be asked as in Section 8.3 regarding the practical meaning and usability of measures other than RAW. Let it just be mentioned that statement " $V_{M}=1$ " or " $V_{M}=0$ " (which is made as a part of formal derivation of formulas for importance measures) may have certain implications on other 
basic events $V_{k}, k=1, \ldots, M$ due to their postulated mutual exclusivity. Evaluation of possible implications would exceed the purpose of this discussion, as well as the space allowed for it. However, let it be mentioned that for the case $V_{M}=1$ (related to RAW) those implications are not relevant for practical PRA applications, since in this case all components in the group are failed by definition. For the case $V_{M}=0$, associated with FC let us just say that FC for this kind of events will generally be very low in practical PRA applications, since $V_{M}$ is usually a low probability event. On the other hand, RAW for $V_{M}$ will often be at the top of the RAW importance list, despite its low probability (or, actually, because of it).

For these reasons, the demonstrations under Example B7 in Appendix $\mathrm{B}$ focus only on RAW importance for this case. It is taken directly from the reported importance measures for the basic event $V_{M}$ and used only to put into perspective the RAW in the case of CCF potential, which was discussed in 8.2.2.

At the end, it may, also, be good to point out that importance discussed in this section is event-related importance, more than componentrelated importance. 


\section{Risk importance of component represented by multiple basic events (failure modes) involved in CCF Groups}

\subsection{Introduction}

In many cases a particular component in a PRA model may be represented by several basic events (each related to specific failure mode) with each of them being involved in some CCF group of basic events. This case will be discussed in this section under the following assumptions:

+ Representative basic events are all input into an "OR" gate. In other words, component's failure logic can be described by expression such as (6-7).

+ Wherever the component is modeled in the overall FT structure, failure model is always the same (i.e. same set of basic events, all input into an "OR" gate).

+ Considered component is one among the group of similar or identical $m$ components which share the potential for the same set of failure modes. In other words, each component is represented by the same kind of the failure logic with corresponding set of basic events. Therefore, each basic event from the set belonging to the considered component is a member of a CCF group consisting of $m$ corresponding basic events.

It should be noted that this kind of the component-level modeling is, actually, normally used to represent failures of components in systems with multiple redundant trains in most of the cases when developing system FT models in a PRA.

One simple example is shown in Figure 9-1 and Figure 9-2. It relates to FT modeling of Motor Driven Pumps A and B in EFW system. Failure logic of each pump consists of two failure modes input into an OR gate, the first one representing failure to start and the second one representing failure to run for a mission time. There are two CCF groups of basic events. The first CCF group is formed by the two basic events representing failure to start of corresponding pumps. Two other basic events, representing failure of corresponding pumps to run, form the second CCF group. 

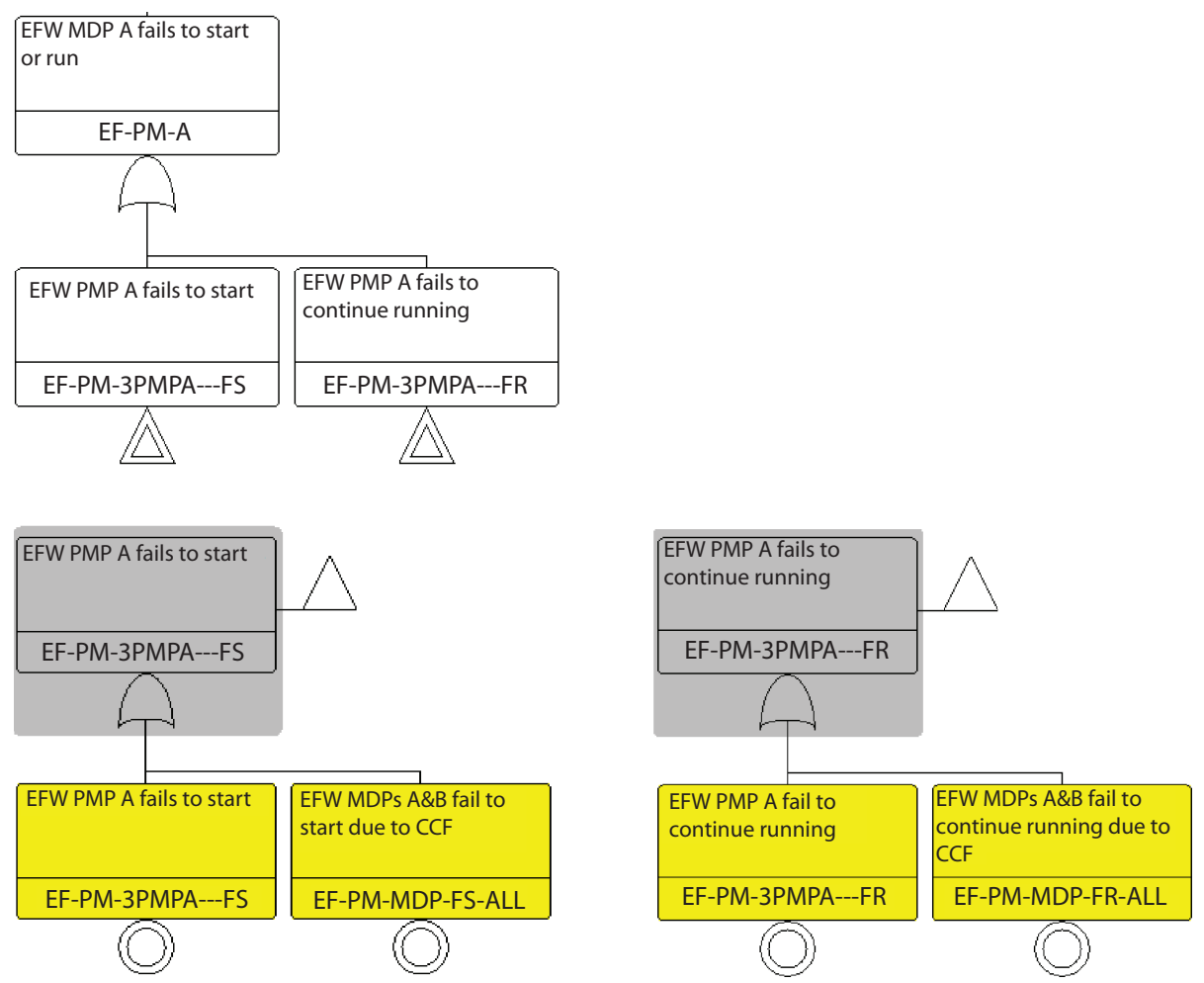

Figure 9-1 Example of component-level failure logic with multiple basic events involved in CCF Groups - EFW motor driven pump (MDP) A.

Failure logic for each pump corresponds to expression (6-7) or (6-8). However, the resulting sub-tree in the FT structure shown in Figure 9-1 ("Pump A") or Figure 9-2 ("Pump B") is not a "FT module" in the sense of Section 6. The term "FT module" as used in Section 6 referred to a component-level sub-tree independent from the rest of the FT structure, which is not the case here because the same CCF events (either failure to start or failure to run) appear in sub-trees for both pumps. Therefore, we will not use asterisk $\left(^{*}\right)$ for denoting the component-level failure, as in (6-7) and (6-8).

In this section we will establish component-level importance measures for the case when considered component is represented in the FT structure in the way discussed above. We will discuss the same three types of failure or unavailability as in Section 8, i.e.: 

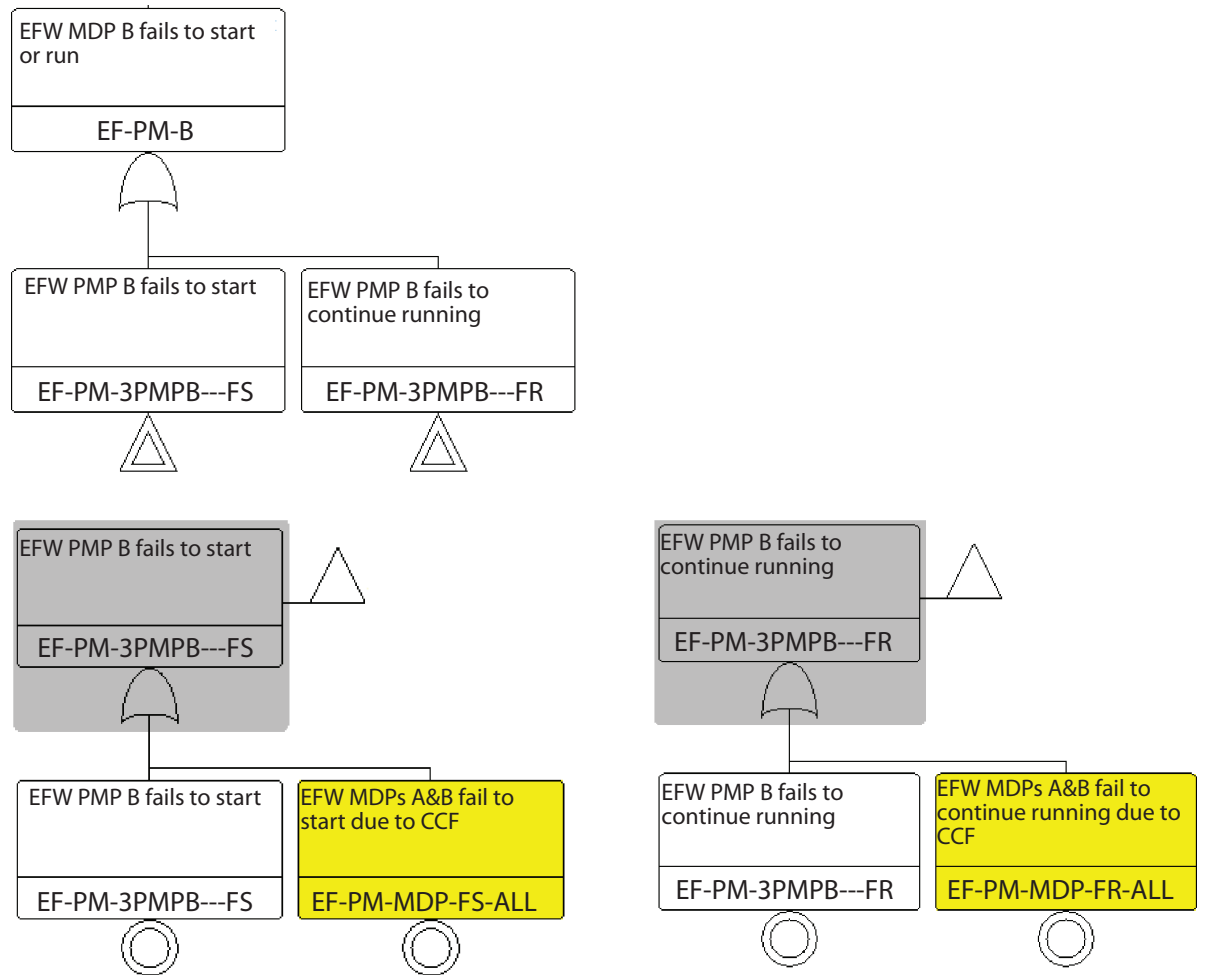

Figure 9-2 Example of component-level failure logic with multiple basic events involved in CCF Groups (continued) EFW MDP B.

1. Failure or unavailability with CCF potential;

2. Unavailability with no impact on CCF (unavailability due to preventive maintenance);

3. Particular CCF event (i.e. failure due to a common cause of specific components).

However, before proceeding, one more assumption will be introduced. According to the above discussion, the component-level failure can be expressed as:

$$
A=\sum_{s=1}^{S} A_{s}
$$

where the terms $A_{s}, s=1, \ldots, S$ represent basic events related to the failure modes such as "pump's failure to start", "pump's failure to run", etc. 
The additional assumption is that these terms are mutually exclusive. While this assumption can be subject to long discussions, in support of its use for this purpose the following can be said:

+ Taking as an example "Pump A" from Figure 9-1 and putting down its logic failure expression as $A=A_{F S}+A_{F R}$ (analogously to (6-8)), the terms $A_{F S}$ and $A_{F R}$ can indeed be interpreted as mutually exclusive for the reasons:

- If pump fails to start, it would not be challenged to run and would, therefore, not fail to run;

+ On the other hand, if pump fails to run, it had to start successfully and, therefore did not fail to start.

+ In the quantification of PRA models the terms $A_{F S}$ and $A_{F R}$ would be treated as independent basic events. This is simply because the quantification algorithms in PRA tools such as RiskSpectrum ${ }^{\circledR}$ and others assume that all basic events involved in the minimal cutsets are independent of each other. If so, then component-level failure probability can be written as:

$$
\begin{aligned}
P(A) & =P\left(A_{F S}\right)+P\left(A_{F R}\right)-P\left(A_{F S} A_{F R}\right) \\
& =P\left(A_{F S}\right)+P\left(A_{F R}\right)-P\left(A_{F S}\right) P\left(A_{F R}\right) .
\end{aligned}
$$

If failure probabilities and failure rates are now taken which are used in the current PRAs (which would show that both $P\left(A_{F S}\right)$ and $P\left(A_{F R}\right)$ are, typically, considerably lower than 1E-02 per demand or per mission, e.g. NUREG/CR-6928), [33], then it can be readily demonstrated that ("rare event") approximation

$$
P(A) \approx P\left(A_{F S}\right)+P\left(A_{F R}\right)
$$

(which corresponds to the assumption on mutual exclusivity) introduces error no larger than a fraction of percent, for a typical pump. Similar argument can be shown for most of the other components modeled in PRA fault trees.

Therefore, by considering the basic events $A_{s}, s=1, \ldots, S$ as mutually exclusive in the derivations which follow one only introduces an 
assumption which is in certain way already present in PRAs and for which some additional supporting arguments may be found.

With stated assumption:

$$
P(A)=\sum_{s=1}^{S} P\left(A_{s}\right)
$$

This time, i.e. for the described case of a component represented by basic events $A_{s}, s=1, \ldots, S$ involved in CCF groups, we will start consideration of component-level importance measures with RAW. Primary reason is that, following the discussions in Section 8, it comes as most intuitive. Additional reason is that, as already mentioned in Section 8, for the last two of the three considered types of failure or unavailability (i.e. preventive maintenance and specific CCF event) other importance measures may not have practical meaning.

\subsection{Failure with CCF potential}

Calculation of the four importance measures for the case with CCF potential is discussed in Sections 9.2.1 through 9.2.4. Two demonstrations are provided in Example B8 in Appendix B.

\subsubsection{RAW}

There is a difference between the case when different failure modes $A_{s}, s=1, \ldots, S$ are involved in CCF groups and the example discussed in Section 6.3 (FT module expressed by logic function (6-8)). When component-level failure was postulated in Section 6.3, it did not matter which of the failure modes actually occurred because the impact on the system and, hence, the likelihood of the top event was the same. This, however, is not so when particular failure modes are involved in CCF groups. The reason is that conditional CCF probabilities for different failure modes generally differ. Taking as an example two redundant motor-driven pumps, it can be seen from the references such as NUREG/CR-5845 [36], or NUREG/CR-5497 [37], that conditional CCF probabilities for failure during operation can be considerably lower (e.g. by a factor) than those for failure on demand. For illustration, let us assume that beta-factor for pump's failure to start is $10 \%$ and betafactor for pump's failure to run is $5 \%$. Let us, then, postulate that first pump has failed. In such a case, then: 
- If the failure came from failure to start, the probability that second pump would also fail is $10 \%$;

+ If, however, the failure came from failure to run during the mission time, the probability that second pump would also fail is $5 \%$.

Each of these failure modes has its own RAW value and they could be considerably different. The component-level RAW value needs to reflect the particular RAW values of all failure modes.

Starting from the most general expression for RAW, (5-4):

$$
I_{R A W}=\frac{P(B \mid A)}{P(B)}=\frac{P(B A)}{P(B) P(A)}=\frac{P\left(B \sum_{s=1}^{S} A_{s}\right)}{P(B) P(A)}
$$

Taking into account the assumption on mutual exclusivity reflected in (9-2) this can further be written as:

$$
I_{R A W}=\frac{\sum_{s=1}^{S} P\left(B A_{s}\right)}{P(B) P(A)}
$$

Upon taking into account $P\left(B A_{s}\right)=P\left(B \mid A_{s}\right) P\left(A_{s}\right)$ and rearranging, one finally obtains:

$$
I_{R A W}=\sum_{s=1}^{S} \frac{P\left(A_{s}\right)}{P(A)} \frac{P\left(B \mid A_{s}\right)}{P(B)}
$$

which can be rewritten in a shorter form as:

$$
I_{R A W}=\sum_{s=1}^{S} H_{s} I_{R A W, s}
$$

In the above expression the first term, $H_{s}$, is conditional probability that failure of $A$ has occurred due to failure mode $A_{s}, s=1, \ldots, S$ :

$$
H_{s}=\frac{P\left(A_{s}\right)}{\sum_{s=1}^{S} P\left(A_{s}\right)}
$$


The second term, $I_{R A W, s}$ represents the RAW value associated with particular failure mode $A_{s}, s=1, \ldots, S$. Each of these particular RAW values can be obtained as described in Section 8 .

Once RAW is known, all other importance measures can be calculated directly.

Note that (9-7) assumes that there is no additional information regarding which of the failure modes $A_{s}, s=1, \ldots, S$ may have caused the failure of the component. In other words, it reflects the designer's point of view (long-term averaged status and risk) rather than that of an evaluator of particular precursor event from the operating experience or evaluator of configuration-specific risk (conditional status and risk). For the calculation of long-term averaged RAW the probabilities in (9-7) would be the probabilities of the corresponding basic events in the base-case PRA model. However, in the case of, for example, a precursor evaluation the likelihood of particular failure modes $A_{s}, s=1, \ldots, S$ can be considerably different based on the observed evidence. (For example, it may be known that pump has actually failed to start rather than to continue running.) For more discussion on these aspects interested readers are directed to check other references, such as already mentioned [27] and [29]. It may be worth mentioning that, for reasons such as these, RAW values calculated by a base-case PRA model can be directly used in precursor risk significance assessment only with due attention being paid to interpretation of observed evidence.

\subsubsection{RRW}

RRW can be calculated directly from its theoretical relation to RAW, (5-9) since it is valid generally:

$$
I_{R R W}=\frac{1-P(A)}{1-P(A) I_{R A W}}
$$

In this case, $P(A)$ represents component-level failure probability expressed by (9-2).

9.2.3 FC

Without going into mathematical formalism, the absolute contribution for the component $A$ can be defined in a corresponding manner to (5-10) and (8-7) as: 


$$
I_{F C}=P\left(\sum_{s=1}^{S} \sum_{k=1}^{M}(C V)_{s, k}\right)
$$

where the terms " $C$ " and " $V$ " have the same meaning as in 8.2.1. Index " $s$ " relates to the numbering of different failure modes, while index " $k$ " relates to numbering of different CCF basic events within particular failure mode. Based on the mutual exclusivity of " $V$ terms" (Section 8.2):

$$
I_{F C}=\sum_{s=1}^{S} I_{F C, s} \quad \text { or } \quad I_{f_{c}}=\sum_{s=1}^{S} I_{f c, s}
$$

where $I_{F C, s}$ or $I_{f c, s}$ refers to a contribution from the failure mode "s", which can be calculated as described in Section 8.

Also, general expression for the top event, (5-3) and (8-3), can be rewritten as

$$
B=L^{\prime \prime}+\sum_{s=1}^{S} \sum_{k=1}^{M}(C V)_{s, k}
$$

and, then, using the same kind of rationale as in Section 5.4 (including the application of "rare event approximation"), it is not difficult to express FC through the RRW by the formula corresponding to (5-18):

$$
I_{F C}=P(B)-\frac{P(B)}{I_{R R W}}, \quad \text { or } \quad I_{f c}=1-\frac{1}{I_{R R W}}
$$

or through RAW, by a formula corresponding to (5-17):

$$
I_{f_{c}}=\frac{I_{R A W}-1}{\frac{1}{P(A)}-1}
$$

In the latest formula, $P(A)$ is calculated by (9-2).

\subsubsection{Reliability importance}

Following the same reasoning as in 8.2 .4 , reliability importance can be calculated directly from RAW and RRW, (5-21), or from FC, (5-22). The probability $P(A)$ is calculated by $(9-2)$. 


\subsection{Unavailability with no impact on CCF}

For the reasons discussed in Section 8.3 we will limit the discussion of risk importance for this type of failure or unavailability on RAW measure. For simplicity, we will use the same kind of convention as in Section 8.3: basic event $V_{s, 1}, s=1, \ldots, S$ will be defined as occurrence of failure mode $A_{s}$ not shared by any other component from the CCF group. (It needs to be noted that now we have $S$ different CCF groups of basic events, i.e. one group of $m$ basic events for each failure mode considered.)

This kind of unavailability of considered component is defined in a way that there is no impact on CCF events involving other components. Therefore, it can be "modeled" as occurrence (setting to "true" status) of any basic event $V_{s, 1}, s=1, \ldots, S$, without any additional impact.

In other words, component-level RAW can be obtained as RAW value of any of the basic events $V_{s, 1}, s=1, \ldots, S$ (which can be read directly from the PRA results). Note that all basic events $V_{s, 1}, s=1, \ldots, S$ will have the same RAW values. This is because their impact, when assumed "true", is limited to the considered component only.

\subsection{Particular CCF event}

The discussion will be, for the reasons discussed in Section 8.4, limited to the RAW values of CCF events involving failures of all $m$ redundant components. In this case we have $S$ common cause failure events which involve all $m$ components, i.e, one such CCF event for each failure mode "s". For convenience (correspondingly to Section 8.4), we will define basic event $V_{s, M}, s=1, \ldots, S$ as, specifically, being occurrence of failure mode $A_{s}$ which is shared by all other redundant components.

By definition, when set to "true" status each of these basic events $V_{s, M}, s=1, \ldots, S$ fails all $m$ components. In other words, $P\left(B \mid V_{s, M}\right)$ is same for every $V_{s, M}, s=1, \ldots, S$, resulting with same RAW value.

Thus, RAW value for any of these events (which can be read directly from PRA results) can be taken as representative RAW for the CCF event involving all $m$ components.

Two demonstrations in Example B8 (Appendix B) include RAW values for the CCF of all $m$ components (this section) and unavailability with no impact on CCF (Section 9.3 above), which are compared against the RAW for CCF potential (Section 9.2). The results are, also, shown in Section 10. 


\section{Demonstrative examples of the component-level importance measure}

A demonstrative example application was carried out using a simplified PRA model to calculate the component-level risk importance measures from the PRA-calculated risk importance measures of the basic events relating to the components. The simplified PRA model used for the demonstrative examples is presented in Appendix B.1. It is based on a pressurized water reactor and is built on RiskSpectrum ${ }^{\circledR}$ PRA computer code. It is pointed out that this model was developed for demonstration of risk importance measures only and should not be related to actual NPP risk.

In a typical PRA computer code only the risk importance measures associated with the basic events are usually provided. As an example, all importance measures which are calculated by the RiskSpectrum ${ }^{\circledR} \mathrm{com}$ puter code are described in Appendix A.

In the example PRA model from Appendix B.1, a selected number of components are focused upon to show the component-level importance measures and their interpretations. Table 10-1 presents a summary of those components. For each component, it also shows the quantitative input which is needed from the RiskSpectrum ${ }^{\circledR}$ PRA model for calculation of component-level importance measures by the formulas described above. This input consists of the probabilities of representative basic events and FC measures for those basic events, as calculated by the RiskSpectrum ${ }^{\circledR}$.

The component-level risk importance measures for those components are presented in Table 10-2. They were obtained in two ways. The first one is by appropriate modification of the PRA model (e.g. to reflect the failed or "perfect" status of the considered component) and its rerunning. This is, actually, the most "exact" way achievable in practice to calculate the importance measures. It is here referred to as "full requantification of PRA model". The results are shown in the third column. The second way to obtain the component-level importance measures is to use the formulas described above. Corresponding results are shown in the fourth column. The details on how the importance measures were, in all cases considered, obtained in both ways can be found in Appendix B.3. As can be seen from the comparison of the values in two columns, the results obtained by the formulas are the same or very close to those 


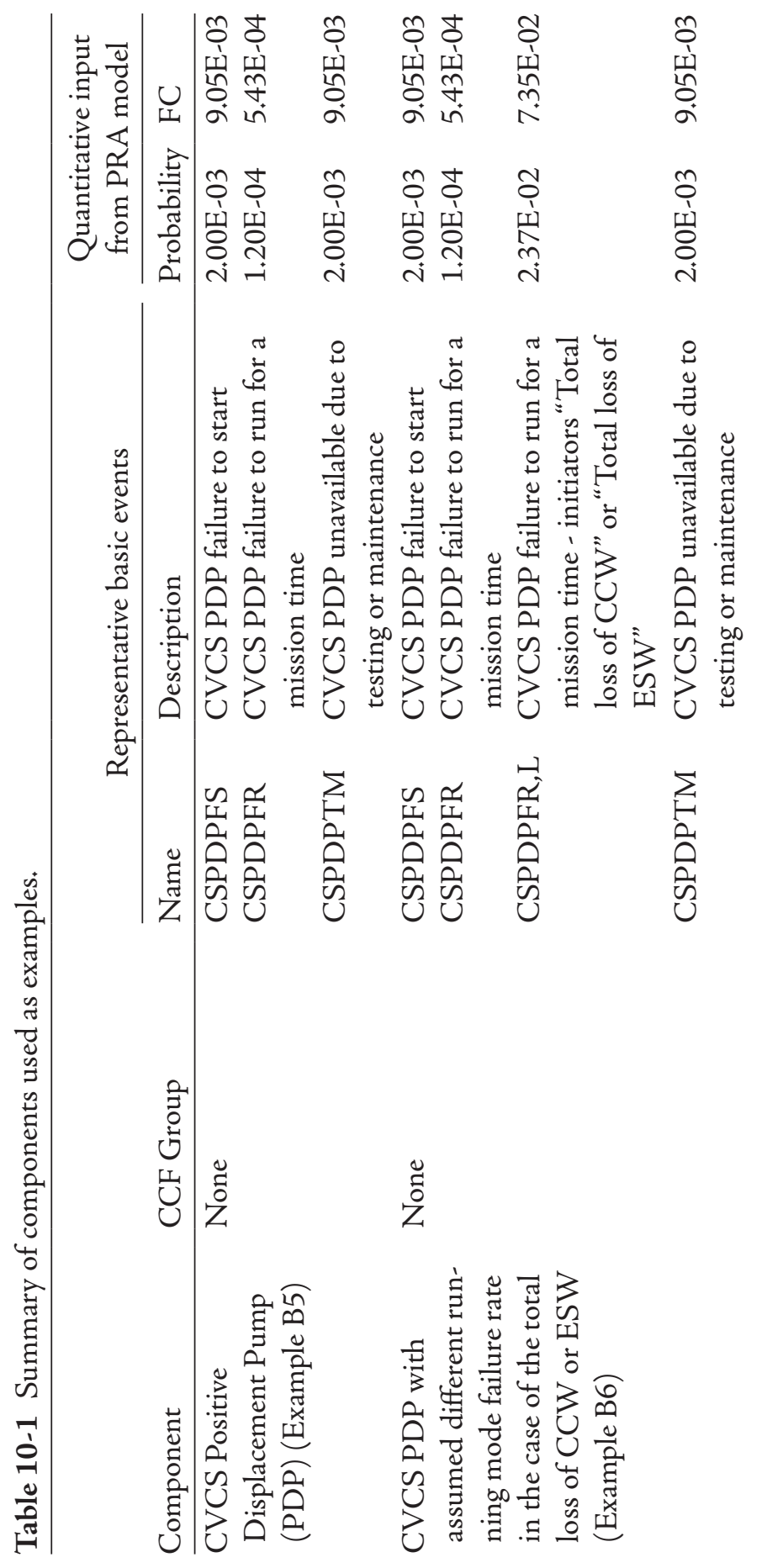


Examples of the Component-Level Importance Measure 81

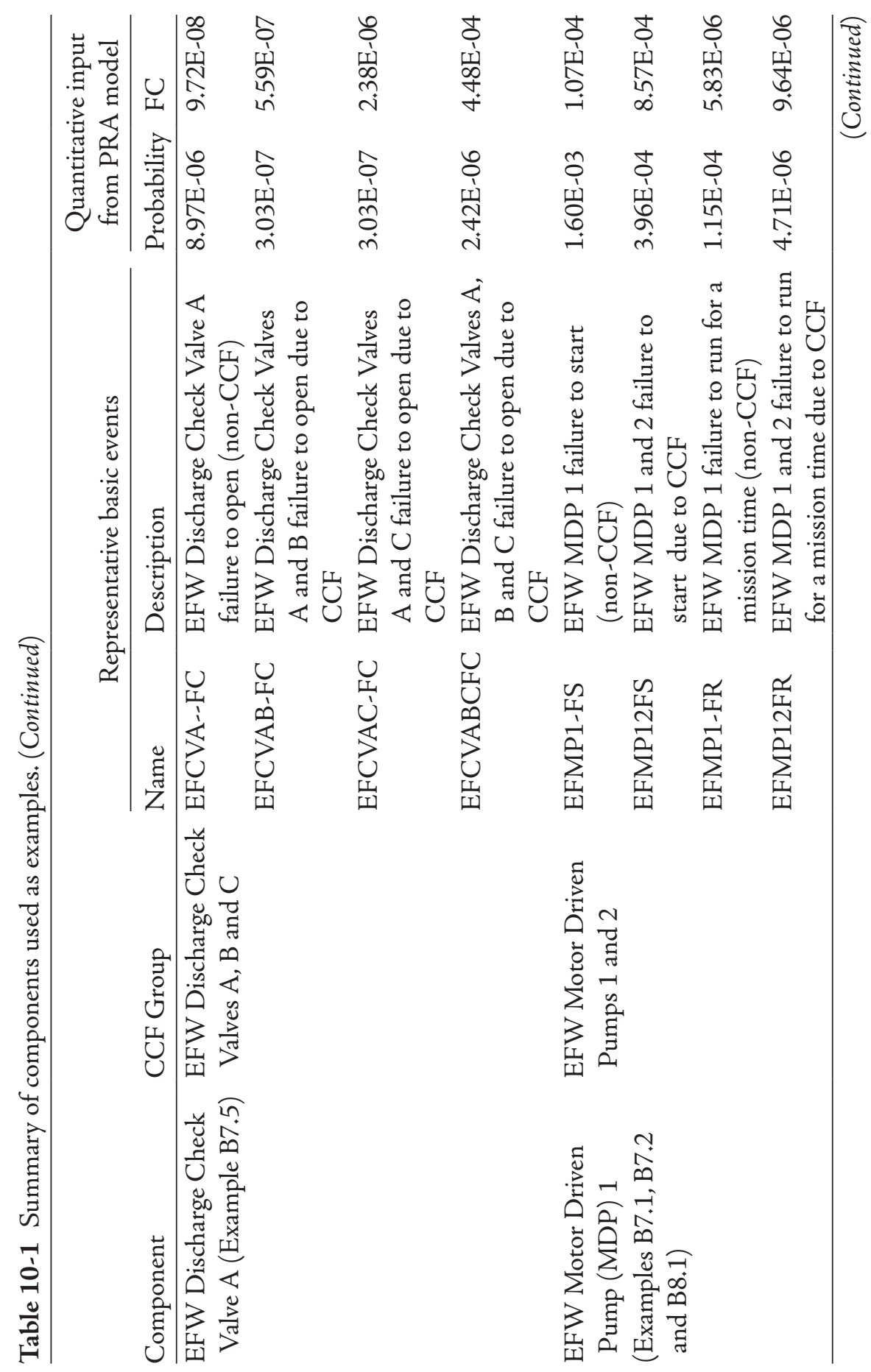


82 Risk Importance Measures in the Design and Operation of NPPs

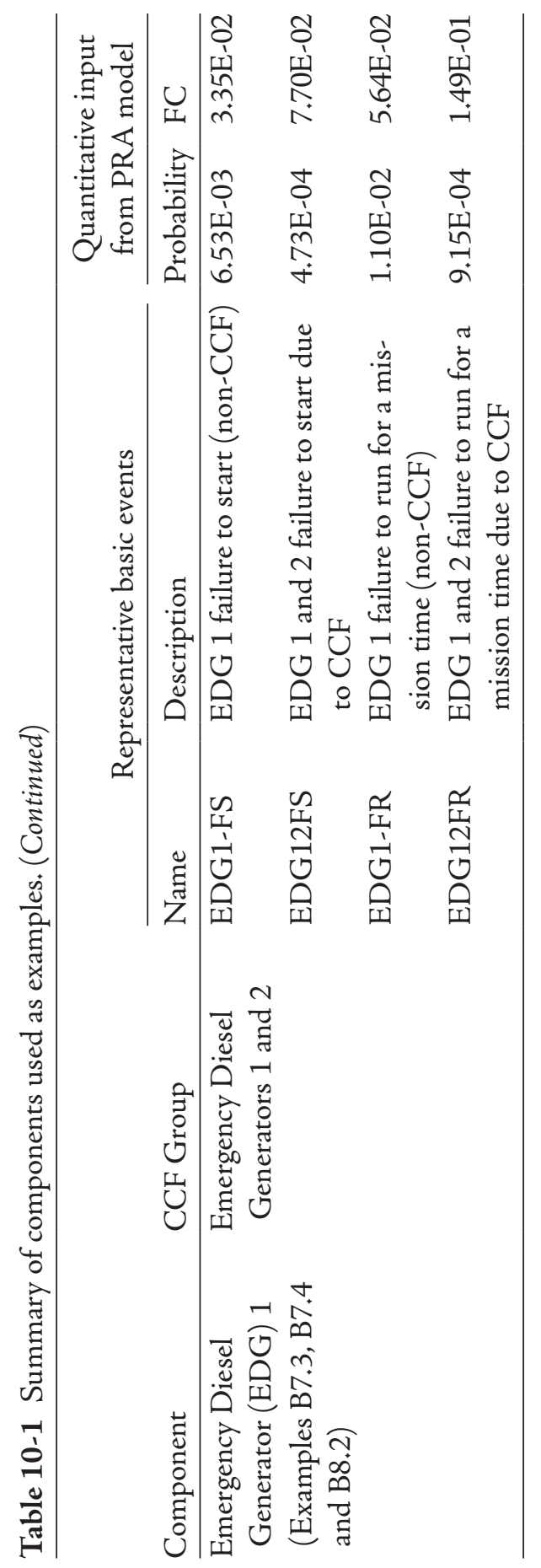


Table 10-2 Summary of calculated component-level importance measures.

\begin{tabular}{|c|c|c|c|}
\hline \multirow[b]{2}{*}{ Component } & \multicolumn{3}{|c|}{ Calculated risk importance measures } \\
\hline & $\begin{array}{l}\text { Importance } \\
\text { measure }\end{array}$ & $\begin{array}{l}\text { By full } \\
\text { PRA model } \\
\text { requantification }\end{array}$ & $\begin{array}{c}\text { By } \\
\text { formulas }\end{array}$ \\
\hline \multirow[t]{4}{*}{ CVCS PDP } & FC & $1.86 \mathrm{E}-02$ & $1.86 \mathrm{E}-02$ \\
\hline & RAW & $5.43 \mathrm{E}+00$ & $5.51 E+00$ \\
\hline & RRW & $1.02 \mathrm{E}+00$ & $1.02 \mathrm{E}+00$ \\
\hline & $\begin{array}{l}\text { Reliability } \\
\text { Importance }\end{array}$ & $1.81 \mathrm{E}-04$ & $1.83 \mathrm{E}-04$ \\
\hline \multirow{4}{*}{$\begin{array}{l}\text { CVCS PDP with } \\
\text { assumed failure rate } \\
\text { in the case of the } \\
\text { total loss of CCW } \\
\text { or ESW }\end{array}$} & $\mathrm{FC}$ & $9.04 \mathrm{E}-02$ & $9.03 E-02$ \\
\hline & RAW & $5.04 \mathrm{E}+00$ & $5.09 \mathrm{E}+00 \mathrm{E}$ \\
\hline & RRW & $1.10 \mathrm{E}+00$ & $1.10 \mathrm{E}+00$ \\
\hline & $\begin{array}{l}\text { Reliability } \\
\text { Importance }\end{array}$ & $1.82 \mathrm{E}-04$ & $1.82 \mathrm{E}-04$ \\
\hline \multirow{4}{*}{$\begin{array}{l}\text { EFW Discharge } \\
\text { Check Valve A }\end{array}$} & FC & $4.45 \mathrm{E}-04$ & $4.51 \mathrm{E}-04$ \\
\hline & RAW & $3.91 \mathrm{E}+01$ & $3.86 \mathrm{E}+01$ \\
\hline & RRW & $1.00 \mathrm{E}+00$ & $1.00 \mathrm{E}+00$ \\
\hline & $\begin{array}{l}\text { Reliability } \\
\text { Importance }\end{array}$ & $1.53 \mathrm{E}-03$ & $1.52 \mathrm{E}-03$ \\
\hline \multirow[t]{4}{*}{ EFW MDP 1} & FC & $9.89 E-04$ & $9.82 \mathrm{E}-04$ \\
\hline & RAW & $1.47 \mathrm{E}+00$ & $1.46 \mathrm{E}+00$ \\
\hline & RRW & $1.00 \mathrm{E}+00$ & $1.00 \mathrm{E}+00$ \\
\hline & $\begin{array}{l}\text { Reliability } \\
\text { Importance }\end{array}$ & $1.89 \mathrm{E}-05$ & $1.89 \mathrm{E}-05$ \\
\hline \multirow[t]{4}{*}{ EDG 1} & FC & $3.16 \mathrm{E}-01$ & $3.16 \mathrm{E}-01$ \\
\hline & RAW & $1.74 \mathrm{E}+01$ & $1.75 \mathrm{E}+01$ \\
\hline & RRW & $1.46 \mathrm{E}+00$ & $1.47 \mathrm{E}+00$ \\
\hline & $\begin{array}{l}\text { Reliability } \\
\text { Importance }\end{array}$ & $6.65 \mathrm{E}-04$ & $6.82 \mathrm{E}-04$ \\
\hline
\end{tabular}




\section{Risk Importance Measures in the Design and Operation of NPPs}

obtained by the full PRA model requantification. Slight differences are attributable to rounding errors and to approximations and truncation process incorporated in the top event probability (frequency) quantification by the PRA model.

These two tables demonstrate the advantage of obtaining the component-level importance measures in deciding the risk significance of components or prioritizing the components in terms of their risk significance. As discussed earlier, in many applications, judgments are needed for components and the user would not have to sort through risk importance of multiple basic events associated with a component; instead, a direct measure for the component could be conveniently used.

Additionally, Table 10-3 focuses on the results for the component-level RAW importance measure for those components which are involved in a CCF group. This is of special interest in many PRA applications. The Table 10-3 shows, for each component, obtained RAW values for the three types of unavailability discussed in Sections 8 and 9. The representative component-level RAW is shown in the middle position of the three values. This is "unavailability or failure with CCF potential" (type 1 in Sections 8 and 9). It reflects an importance of unavailability (or failure) of a component which may (depending on its cause) have implications on the status of other components in the CCF group. On the left side of the representative RAW shown is the RAW for the case when the component is taken out of service for preventive maintenance, without any implications on the status of other components in the CCF group (type 2 in Sections 8 and 9). On the right side of the representative RAW shown is the RAW for the unavailability due to a CCF of all components in the group (particular case of type 3 in Sections 8 and 9). The details on the calculations can be found in the corresponding examples presented in Appendix B.3.

As shown in the Table 10-3, for all cases:

$$
\begin{aligned}
& \text { RAW (Prev. maintenance })<\text { RAW }(\text { CCF potential })< \\
& \text { RAW }(\text { CCF of all components })
\end{aligned}
$$

The representative component-level RAW (i.e. with CCF potential) is higher than the RAW associated with preventive maintenance. This is expected and is because the representative component-level RAW is adjusting for the common-cause contribution. This increase in the 
Table 10-3 Summary of calculated component-level RAW for three different types of unavailability of components involved in a CCF Group.

\begin{tabular}{cccc}
\hline & \multicolumn{3}{c}{ RAW } \\
\cline { 2 - 4 } & $\begin{array}{c}\text { Unavailability } \\
\text { due to } \\
\text { preventive } \\
\text { maintenance }\end{array}$ & $\begin{array}{c}\text { Unavailability } \\
\text { or failure with } \\
\text { CCF potential }\end{array}$ & $\begin{array}{c}\text { Unavailability } \\
\text { due to CCF of } \\
\text { all components } \\
\text { in the group }\end{array}$ \\
\hline $\begin{array}{cccc}\text { EFW Discharge } \\
\text { Check Valve A }\end{array}$ & 1.07 & 38.6 & 185 \\
EFW MDP 1 & 1.07 & 1.46 & 3.16 \\
EDG 1 & 6.03 & 17.5 & 161 \\
\hline
\end{tabular}

component-level RAW depends on the CCF contribution. On the other hand, it is shown that the representative component-level RAW is significantly lower than the RAW for the CCF event of all components involved in the CCF group. This also comes as expected because the RAW for the total CCF event does not represent the importance of the considered component alone. Rather, it represents the collective importance (due to this particular cause) of unavailability of all components in the group.

Generally, changes in the different risk measures from a basic event for the component to the component-level are different. For example, considering the information from Tables B-7 and B-11 in Appendix B, one can see that: RAW for EDG 1 "failure to start" is 6.03 and component level RAW for EDG 1 is 17.5; FC for EDG 1 "failure to start" is 3.35E-02 and the component-level FC is 3.16E-01; RRW for EDG 1 "failure to start" (considering $R R W=\frac{1}{1-F C}$ ) is 1.03 and the componentlevel RRW for EDG 1 is 1.47. This is expected and consistent with the understanding of the measures.

The use of the component-level risk importance measures can be considered more appropriate as they would take into consideration different failure modes of the component, including the common-cause failures, in an integrated manner removing the judgment process of the user. This would simplify the SSC categorization process and make PRA results more understandable and directly usable. 


\section{Limitations of risk importance measures}

As discussed before, risk importance measures are used in many applications to address issues relating to regulation and operation of NPPs. As being discussed in this monograph, these measures provide useful inputs and make the PRA models of NPPs present results that are directly applicable for the issues to be addressed. In these applications, the information obtained from these measures should be viewed in the context of their limitations some of which are discussed here. The last two points (i.e. 6 and 7) provide a discussion on some aspects and limitations of methods specifically considered in previous sections in this monograph.

1. PRA quality and scope

A PRA with an appropriate capability and quality is needed for calculation of risk importance measures for the SSCs. In many applications where risk importance measures have been used, PRA quality has been discussed and emphasized. Without a quality PRA that includes appropriate modeling and applicable data, the risk importance measures can have large uncertainties associated with them making them unusable. In some situations, PRA scope may not cover all internal and external hazards. Then, adequate considerations in addition to those obtained from the risk importance measures should be given to address the hazards not considered in defining the SSCs into safety-significant and non-safety-significant categories for different applications.

2. Varying levels of uncertainty and different degrees of conservatism in the spectrum of risk contributors

When a PRA is available addressing full-scope of hazards as well as the shutdown operation mode, it is expected that the level of uncertainty and the degrees of conservatism in the different risk contributors (e.g., fire, seismic, at-power vs shutdown) will be different. Consideration of a single risk importance measure calculated by an integrated model is a useful input, but may mask contribution of individual risk contributors that may need consideration. Care should be taken in use of such importance measures. Usually, separate evaluations are conducted for the contributors along with an integrated measures and a qualitative consideration by a group of experts from different disciplines are used. In such a way, the varying levels of uncertainty and degrees of conservatism are given due consideration. 


\section{Adequate modeling of the SSCs in the PRA}

PRA model for an NPP includes many SSCs and the risk importance measures obtained using the PRA model includes the basic events for a long list of components. In many cases, the user evaluates the list to make judgment about the SSCs. It may be tempting to judge that a component is not risk-significant because any basic event belonging to the component does not appear in the list. It is possible that the SSC did not appear in the list is because the SSC was not modeled or its appropriate failure mode was not included. Use of an expert panel or a review of the PRA model can address such situations.

Additional point for consideration is that certain SSC may be included in the PRA model implicitly, e.g. as a part of initiating event (IE). Implicit contribution may be possible to address, for example, by expressing the importance of SSC with respect to the PRA element considered (e.g. FC of SCC with regard to the IE frequency) and combining it with the importance of the PRA element itself (e.g. FC of an IE).

4. Truncation level used to calculate risk importance measures

A PRA assesses a large number of accident sequences to calculate CDF and LERF. To manage the process, accident sequences cutsets are truncated to estimate accident sequence frequencies, CDF and LERF. Appropriate level for truncation should be used to avoid any underestimation. This issue becomes particularly relevant when conditional risk calculations, as needed for risk importance measures, are performed. In general, the selected truncation level should support an overall CDF/ LERF value that has converged and the unaccounted for frequencies are sufficiently low to provide confidence that the calculated risk importance measures are accurate.

Some guidance in this regard is available in different documents. NEI 00-04 [17], provides the following guidance. It recommends a CDF (or LERF) truncation level of five orders of magnitude below the baseline CDF (or LERF) when calculating the F-V risk importance measure. For example, if the internal events, full power CDF baseline value is $1 \mathrm{E}-5 / \mathrm{yr}$, a truncation level of at least $1 \mathrm{E}-10 / \mathrm{yr}$ is recommended.

When RAW risk importance measure is calculated which requires calculation of condition CDF/LERF for the component being unavailable, the truncation level should be given attention. If it is calculated by 
the full re-solution of the PRA model then the truncation level should not significantly affect the calculation A truncation level of $1 \mathrm{E}-9 / \mathrm{yr}$ is considered reasonable by NEI 00-04 [17]. If the model relies on presolved set of cut sets to calculate the CDF, then the RAW values may be underestimated. First of all, it must be checked that all safety functions are being represented and that the SSC in question is not being truncated out in the pre-solved cutsets. Sensitivity analyses may need to be performed to identify the appropriate truncation level to use to ensure that the CDF and LERF calculations are sufficiently accurate to obtain accurate risk importance measures.

\section{Defense in depth and safety margin considerations}

In making decisions using risk importance measures, due consideration should be given to fundamental principles for ensuring safety in NPPs. As discussed earlier, these are referred to as deterministic considerations that have proven effective in ensuring safety. In defining an SSC to be low safety significance, it is appropriate to confirm that defensein-depth and safety margins are preserved in addition to the results of risk importance measures.

The defense-in-depth assessment ensures that adequate redundancy and diversity will be retained in design basis accidents. This assessment evaluates the SSC functions with respect to core damage mitigation, early containment failure/bypass, and long-term containment integrity. Existing safety margins for SSCs arising from the design technical and functional requirements should also not be compromised. A systematic consideration of defense-in-depth and safety margin is discussed in Regulatory Guide 1.174 [38], and NEI 00-04 [17].

\section{Complex models of a component in FTs in a PRA}

One can easily imagine more and more complex models of a "component" in FTs in a PRA, such as: different FT modules (sub-trees) applying in different ET sequences, which share some basic events (i.e. they are not independent) and some of those basic events are involved in CCF groups, etc. However, it is believed that calculation of component-level importance measures for such complex cases can be made by combining the principles and formulas from the cases discussed in the monograph.

To make an outline of an approach toward determining the componentlevel importance measures in a general case we can offer the following points: 
+ The four importance measures considered here are related. If one of them is known and if a component-level failure probability $P(A)$ is known, the other three can be directly calculated. (This process involves some approximations, as discussed.)

- Starting with FC appears to be most convenient. This is so because component-level FC can, usually, be expressed (approximated) in the form of a sum of more elementary FCs (e.g. FCs for basic events) which can be taken from PRA results.

- Assuming that FC is known, the question to be addressed is whether the effective component-level failure probability $P(A)$ can be determined. This may not be straightforward, as some of the examples have shown, and may require detailed knowledge of PRA logic. However, if $P(A)$ can be determined then all three other measures can be directly obtained by formulas (e.g., case B) in the table in Section 12).

The process can, also, start with a measure other than FC, if more convenient.

\section{Limitations of methods discussed}

For the end of this section, we wanted to recapitulate the limitations and main assumptions involved in the methods discussed in this monograph. The very first one, introduced in Section 5.1 is that probability of a top event, $P(B)$, as designated throughout the monograph, can be taken as a representative or surrogate for the quantitative risk $(R)$. Then, the next one, postulated in same Section 5.1 is that this top event (e.g. reactor core damage) is expressed in terms of logic sum of minimal combinations of failures (minimal cutsets). This basically means that discussions are limited to PRAs developed by the "fault tree linking" approach, as emphasized in 5.1. There are other PRA approaches, sucn as "event tree linking" (with the event trees in which function events in sequences are made mutually independent by use of initial/boundary conditions and states) or binary decision diagrams (BDDs) wich were not discussed in this monograph. This was so because it could not have been done without "monograph" becoming a "much larger book", which was not possible due to different practical reasons. (We, however, still do believe that many of the discussions are applicable to and can be used with other approaches.)

The above assumption made it, in turn, possible to express the top event by the general logic form $B=L+C A,(5-3)$, which, under couple 
of other approximations enabled establishing the relation between FC and RAW, (5-17), which is one of the key points for the whole discussion. Mentioned approximation were explained in Section 5.4 and a claim was made that they would not make a relevant difference in practical PRA applications as long as the failures involved in minimal cutsets are low probability events. This claim was explored in several examples in Appendix B which have, indeed, shown that it would not make a relevant difference with a simplified PRA model used in those exercises, described in B.1. (The simplified PRA model was using failure probabilities and initiator rates which are in the range of those used in the PRAs conducted for operating NPPs.)

The question which can be immediately raised is what if not all basic events involved in MCSs are low probability events, i.e. what kind of impact would it have on calculated importance measures. (The "question within the question" could be what it actually means to say "low probability events". Without going into a long discussion, let the following explanation be offered: those are such events which, when the top event probability calculated, would produce a reasonably small difference between the results obtained by the "Mincut Upper Bound" and $2^{\text {nd }}$ order approximation in the inclusion-exclusion principle. The example of what it means "reasonably small difference" can be several percent or up to $10 \%$, rather than a difference by a factor.) The above question does not have a straightforward and an easy answer and it is, apparently, related to the limits or boundaries of the PRA approach with "fault tree linking" and a single list of minimal cutsets for the top event. Once that "non-low probability failure events start to be introduced into a PRA model on a large scale their complements (i.e. success events) would also need to be included into the combinations of events. Explicit presentation of complements in combinations of (basic) events opens many other questions and, ultimately, leads to other PRA approaches some of which were mentioned above and were not addressed in this monograph.

As a last remark in this section let it be pointed out that discussion of importance measures in the monograph was focused on the base case PRA models and results which reflect long-term averaged risk, rather than on the applications which reflect conditional risk such as configuration risk monitoring. Direct use of risk measures such as RAW for configuration risk monitoring (i.e. to directly calculate conditional risk measures), although possible in principle, should be done with due 
caution in order not to neglect condition-specific aspects (which may, for example, include restorability of equipment out of service due to preventive maintenance). Those additional aspects relevant for particular PRA applications (as compared to the base case model) were not considered in the discussions, with exception of some brief mentions in the sections devoted to failure modes or components involved in a CCF group. 


\section{Summary}

In this monograph, we have presented the risk importance measures calculated in the PRAs of NPPs and used in many applications. We have discussed the derivations of these measures and have presented relations among them using basic probabilistic principles. We have shown how each measure correlates with others and knowledge of one measure can be used to obtain other measures.

In reviewing the risk importance measures currently used, we have presented the concept of a "component-level" risk importance measure. The "component-level" risk measures have been analytically derived from the basic event probabilities and measures.

Using a simple PRA model and RiskSpectrum ${ }^{\circledR}$ computer code, we carried out calculations for the different risk importance measures including the "component-level" risk importance measures. We were able to compare the values of the measures obtained directly by the derived equations with those obtained on the basis of full PRA model requantification. The results are included and show the validity of the "component-level" measures for the cases discussed here.

The behavior of the "component-level" measures studied from the demonstrative examples presented here is also satisfactory and points to the usefulness of these measures. Ranking of the components based on the component-level measure would, also, be appropriate based on consideration of the common-cause failure contribution which is desirable and encompasses different roles of a component.

The calculation of these component-level measures is relatively simple and straightforward. The input is readily available: it consists of basic event probabilities included in the PRA model and basic event importance measures reported by its quantification. The "componentlevel" measures could be provided along with other basic event importance. These measures would provide additional useful information in a PRA application. As discussed, the availability of such information can make the use of PRA straightforward, reducing the need for judgment of the analyst.

Below we summarize, in tabular form, expressions for and calculations of the four importance measures for the cases considered in this monograph (Sections 5 through 9). 


\section{Risk Importance Measures in the Design and Operation of NPPs}

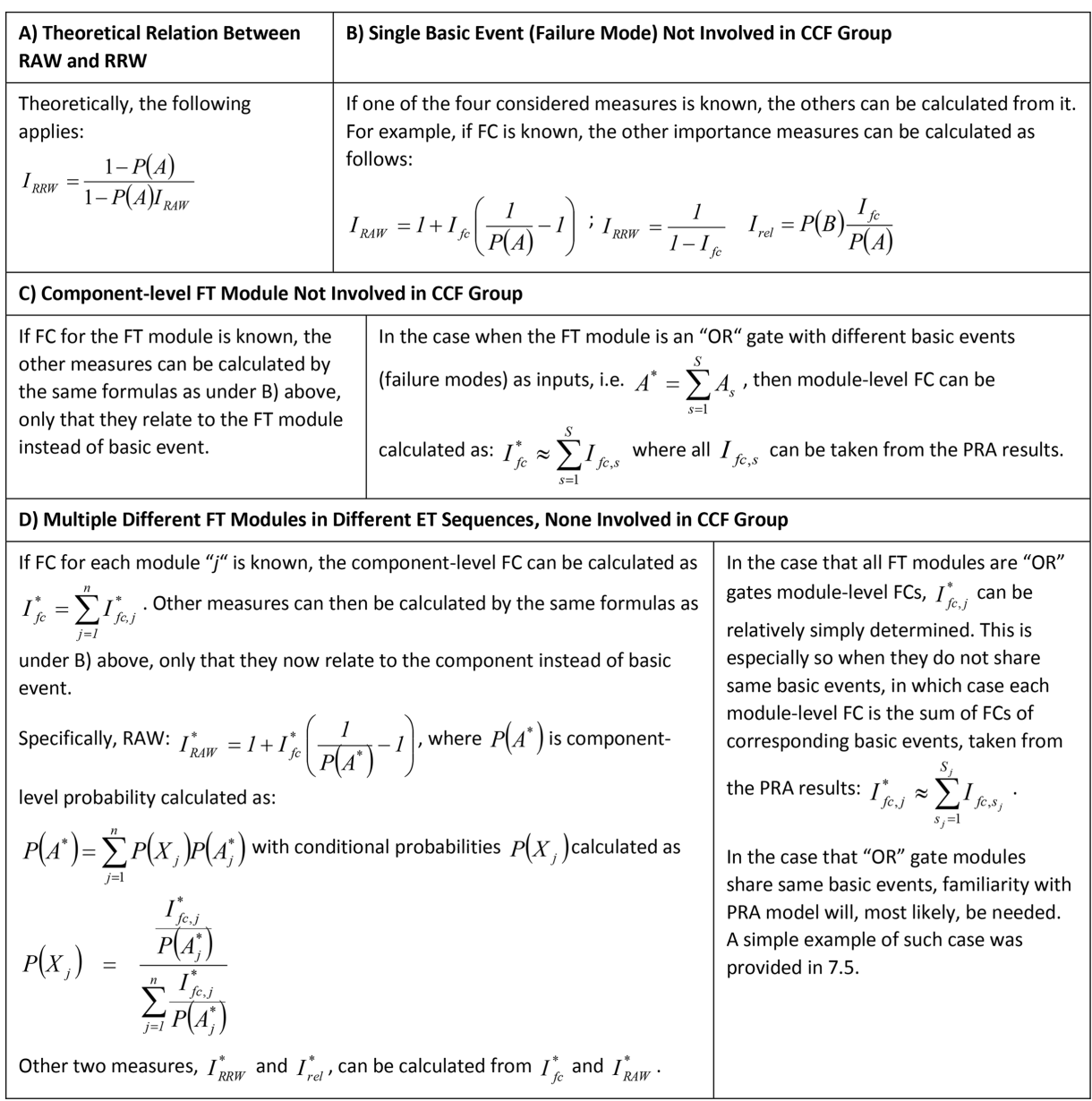




\section{E) Component Represented by Single Basic Event (Failure Mode) Involved in CCF a Group}

Three cases are of interest: 1) Failure or unavailability with CCF potential; 2) Unavailability with no impact on CCF (preventive maintenance); 3) CCF event involving all components.

Failure mode $A$ involved in CCF group of size $m$ is represented by $M=2^{m-1}$ basic events: $A=\sum_{k=1}^{M} V_{k}$ which are shown in minimal cutsets and importance list in the PRA model. For convenience, $V_{1}$ is defined as failure which is not shared with any other component in the group and $V_{M}$ as failure shared with all components in the group.

Case 1): Component-level FC can be calculated as $I_{f c}=\sum_{k=1}^{M} I_{f c, k}$ where $I_{f c, k}$ represents FC of CCF event $V_{k}$ which can be obtained directly from PRA results.

With FC known, other measures can be calculated by the same formulas as under B) above, only that they now relate to the component instead of basic event.

Specifically, component level RAW is calculated as $I_{R A W}=1+I_{f c}\left(\frac{1}{P(A)}-1\right)$ where $P(A)$

represents component-level probability $P(A)=\sum_{k=1}^{M} P\left(V_{k}\right)$.

Case 2) and 3):

All importance measures can be directly obtained as measures for the basic events $V_{1}$ and $V_{M}$, respectively. However, the focus really is on RAW. (Practical meaning and usability of other measures may be questionable.)

Other two measures, $I_{R R W}$ and $I_{r e l}$, can be calculated from component-level $I_{f c}$ and $I_{R A W} \cdot$

Note: In coherent systems: RAW (Case 2) < RAW(Case 1) < RAW(Case 3, all componente). (As an order-of-magnitude approximation: RAW(Case 1) is expected to be in the range of geometric mean of the other two.)

F) Component Represented by Multiple Basic Events (Input to an "OR" Gate) Involved in CCF Groups

Of interest are the same three cases as under $\mathrm{E}$ ) above. Component $A$ is in FT structure presented as $A=\sum_{s=1}^{S} A_{s}$ where $A_{s}$ is particular failure mode which is presented in terms of CCF basic events as under E) above.

Case 1): Calculation can start with component-level RAW: $I_{R A W}=\sum_{s=1}^{S} H_{s} I_{R A W, s}$ where $I_{R A W, s}$ is RAW for failure mode " $s$ ", calculated as under $\mathrm{E}$ ) and $H_{s}$ conditional probability

$H_{s}=P\left(A_{s} \mid A\right) \approx \frac{P\left(A_{s}\right)}{\sum_{s=1}^{S} P\left(A_{s}\right)}$. With RAW known, all other measures can be calculated by formulas as under B) above.

Specifically, component-level FC can be calculated as $I_{f_{c}}=\frac{I_{R A W}-1}{\frac{1}{P(A)}-1}$, where $P(A) \approx \sum_{s=1}^{S} P\left(A_{s}\right)$. Case 2) and 3): RAW value of basic event $V_{1, s}$ is same for any "s" and same applies for $V_{M, s}$. Thus, any $V_{1, s}$ and any $V_{M, s}$ can be taken as representative for a component.

Other two measures, $I_{R R W}$ and $I_{r e l}$, can be calculated from component-level $I_{f c}$ and $I_{R A W}$. Note: Calculation can go, also, the other way: component-level FC can first be calculated as $I_{f c}=\sum_{s=1}^{S} I_{f c, s}$ where $I_{f c, s}$ is FC for failure mode "s", calculated as under E). With FC known, all other measures can be calculated by formulas as under B) above.

Same "component-level" note applies as under E) above. 


\section{References}

[1] Severe Accident Risks: An Assessment for Five U.S. Nuclear Power Plants-Final Summary Report, NUREG-1150, U.S. NRC, 1990.

[2] U.S. NRC Regulatory Guide 1.70, Standard Format and Content of Safety Analysis Reports for Nuclear Power Plants (LWR Edition), Revision 3, as reviewed on September 2014.

[3] U.S. NRC Regulatory Guide 1.26, Quality Group Classifications and Standards for Water-, Steam-, and Radioactive-WasteContaining Components of Nuclear Power Plants, Revision 1, September 1974.

[4] U.S. NRC Regulatory Guide 1.26, Quality Group Classifications and Standards for Water-, Steam-, and Radioactive-WasteContaining Components of Nuclear Power Plants, Revision 4, as reviewed on March 2013.

[5] 10CFR50.55a Codes and standards, 36 FR 11424, Jun. 12, 1971 with last amendment at 80 FR 45843, Aug. 3, 2015.

[6] NUREG-0800 Standard Review Plan for the Review of Safety Analysis Reports for Nuclear Power Plants: LWR Edition, Section 3.2.2 System Quality Group Classification, U.S. NRC, Draft Rev. 3, August 2015.

[7] NEI 97-04, Revised Appendix B, Guidance and Examples for Identifying 10 CFR 50.2 Design Bases, Nuclear Energy Institute, November 2000 (http://adams.nrc.gov/wba/, Accession No.: ML003771698, as of July 15, 2016).

[8] U.S. NRC Regulatory Guide 1.186, Guidance and Examples for Identifying 10 CFR 50.2 Design Bases, as reviewed on June 2011.

[9] Fullwood, R. R., Hall, R. E., Probabilistic Risk Assessment in the Nuclear Power Industry, Fundamentals and Applications, Pergamon Press, 1988.

[10] Henley, E. J., Kumamoto, H., Reliability Engineering and Risk Assessment, Prentice-Hall, Inc., 1981.

[11] Barlow, R. E., Proschan, F., Statistical Theory of Reliability and Life Testing: Probability Models, Holt, Rinehart and Winston, Inc., 1975. 
98 Risk Importance Measures in the Design and Operation of NPPs

[12] Vesely, W. E. et al., Measures of Risk Importance and Their Applications, NUREG/CR-3385, Battelle Columbus Laboratories/ U.S. NRC, 1983.

[13] Vesely, W. E. et al., Fault Tree Handbook, NUREG-0492, U.S. NRC, 1981.

[14] Papazoglou, I. A. et al., Probabilistic Safety Analysis Procedures Guide, NUREG/CR-2815, Brookhaven National Laboratory/ U.S. NRC, 1984.

[15] ASME/ANSRA-Sa-2009,Addenda to ASME/ANSRA-S-2008 Standard for Level 1/Large Early Release Frequency Probabilistic Risk Assessment for Nuclear Power Plant Applications, An American National Standard, The American Society of Mechanical Engineers, 2009.

[16] 10CFR50.69, Risk-Informed Categorization and Treatment of Structures, Systems and Components for Nuclear Power Reactors, 69 FR 68047, Nov. 22, 2004.

[17] NEI 00-04, 10 CFR 50.69 SSC Categorization Guideline, Revision 0, Nuclear Energy Institute, July 2005 (http://adams .nrc.gov/wba/, Accession No.* ML052900163, as of July 15, 2016).

[18] U.S. NRC Regulatory Guide 1.177, An Approach for PlantSpecific, Risk-Informed Decisionmaking: Technical Specifications, Revision 1, May 2011.

[19] 10CFR50.65, Requirements for Monitoring the Effectiveness of Maintenance at Nuclear Power Plants, 56 FR 31324, July 10, 1991 with last amendment at 72 FR 49501, Aug. 28, 2007.

[20] U.S. NRC Regulatory Guide 1.160, Monitoring the Effectiveness of Maintenance at Nuclear Power Plants, Revision 3, May 2012.

[21] NUMARC 93-01, Industry Guideline for Monitoring the Effectiveness of Maintenance at Nuclear Power Plants, Revision 4A, Nuclear Energy Institute, April 2011 (http://adams.nrc.gov/wba/, Accession No.: ML11116A198, as of July 15, 2016).

[22] Reactor Oversight Process, NUREG-1649, Revision 5, U.S. NRC, February 2014.

[23] NRC Inspection Manual Inspection Procedure 71111, Reactor Safety-Initiating Events, Mitigating Systems, Barrier Integrity, Issue Date $10 / 28 / 11$. 
[24] Dube, D. A. et al, Independent Verification of the Mitigating Systems Performance Index (MSPI) Results for the Pilot Plants, NUREG-1816, U.S. NRC, February 2005.

[25] NEI 99-02, Regulatory Assessment Performance Indicator Guideline, Nuclear Energy Institute, August 31, 2013 (http://adams .nrc.gov/wba/, Accession No: ML13261A116, as of July 15, 2016).

[26] Cheok, M. C., Parry, G. W. and Sherry, R. R., Use of Importance Measures in Risk-Informed Regulatory Applications, Reliability Engineering and System Safety 60 (1998) 213-226, Elsevier Science Limited, 1998.

[27] Smith, C. L., Calculating Conditional Core Damage Probabilities for Nuclear Power Plant Operations, Reliability Engineering and System Safety 59 (1998) 299-307, Elsevier Science Limited, 1998.

[28] Kim, K., Kang, D. I. and Yang, J.-E., On the Use of the Balancing Method for Calculating Component RAW Involving CCFs in SSC Categorization, Reliability Engineering and System Safety 87 (2005) 233-242, Elsevier Ltd., 2005.

[29] Bäckström, O., Krcal, P. and Wang, W., Two interpretations of the risk increase factor definition. Risk, Reliability and Safety: Innovating Theory and Practice, Edited by Lesley Walls, Matthew Revie and Tim Bedford, Taylor \& Francis Group, 6000 Broken Sound Parkway NW, Suite 300, Boca Raton, FL 33487-2742 CRC Press, pages 2816-2822, 2016.

[30] Wall, I. B, and Worledge, D. H., Some Perspectives on Risk Importance Measures, Proceedings of the International Topical Meeting on Probabilistic Safety Assessment: PSA '96: "Moving toward Risk-Based Regulation”, September 29-October 3, 1996, Park City, Utah (general chair: James A. Lake), American Nuclear Society, La Grange Park, IL, USA, pages 203-207, 1996.

[31] Poloski, J. P. et al., Rates of Initiating Events at U.S. Nuclear Power Plants: 1987-1995, NUREG/CR-5750, INEEL/U.S. NRC, 1999.

[32] Martorell, S., Serradell, V. and Verdu, G., Safety-Related Equipment Prioritization for Reliability Centered Maintenace Purposes Based on a Plant Specific Level 1 PSA, Reliability Engineering and System Safety 52 (1996) 35-44, Elsevier Science Limited, 1996. 
[33] Eide, S.A. et al., Industry-Average Performance for Components and Initiating Events at U.S. Commercial Nuclear Power Plants, NUREG/CR-6928, Idaho National Laboratory/U.S. NRC, February 2007.

[34] Vesely, W. E., Supplemental Viewpoints on the Use of Importance Measures in Risk-Informed Regulatory Applications, Reliability Engineering and System Safety 60 (1998) 257-259, Elsevier Science Limited, 1998.

[35] NUREG/CR-4780, Procedure for Treating Common Cause Failures in Safety and Reliability Studies, Pickard, Lowe and Garrick, Inc., Prepared for US NRC - EPRI, 1988/1989.

[36] NUREG/CR-5845, Guidelines on Modeling Common Cause Failures in Probabilistic Risk Assessment, INEEL, University of Maryland, Prepared for US NRC, 1998.

[37] NUREG/CR-5497, Common Cause Failure Parameter Estimations, INEEL, University of Maryland, Prepared for US NRC, 1998.

[38] U.S. NRC Regulatory Guide 1.174, An Approach for Using Probabilistic Risk Assessment in Risk-Informed Decisions on Plant-Specific Changes to the Licensing Basis, Revision 2, 2011.

[39] RiskSpectrum ${ }^{\circledR}$ Analysis Tools Theory Manual, Version 3.2.1, Scandpower AB 1984-2012. 


\section{Appendix A. Example from practice-calculation of risk importance measures by a PRA computer code}

This section describes, as an example "from the practice", how the risk importance measures for basic events are calculated by RiskSpectrum ${ }^{\circledR}$. Similar examples can be found with other PRA tools. RiskSpectrum ${ }^{\circledR}$ calculates the following importance measures for the basic events [39]: "Fussel-Vesely importance", "risk decrease factor (RDF)", "fractional contribution", "risk increase factor (RIF)". (The quotation marks are used to point out RS terminology+) Additionally, "sensitivity calculations" are done which can be related to the reliability importance as discussed in this monograph.

Using the symbols from the Theory Manual [39], the "Fussel-Vesely importance" for the basic event " $i$ " is calculated as:

$$
I_{i}^{F V}=\frac{Q_{\text {TOP }}(\text { MCS including } i)}{Q_{\text {TOP }}}
$$

where $Q_{T O P}$ refers to top event probability and the term $Q_{\text {TOP }}($ MCS including $i)$ refers to the top event probability based only on the minimal cutsets which include basic event " $i$."

The RDF (which corresponds to the RRW) is calculated as:

$$
I_{i}^{R}=\frac{Q_{T O P}}{Q_{T O P}\left(Q_{i}=0\right)}
$$

where $Q_{i}$ refers to the probability of basic event " $i$." The term $Q_{T O P}\left(Q_{i}=0\right)$ represents the top event probability recalculated with probability $Q_{i}$ set to zero.

The "fractional contribution" is calculated from RDF as:

$$
I_{i}^{F}=1-\frac{1}{I_{i}^{R}}
$$

It is noted that this equation corresponds to (5-18). Basically, the measures "Fussel-Vesely importance" and "fractional contribution", as applied to the basic events, have the same meaning. They are distinguished because RiskSpectrum ${ }^{\circledR}$ calculates them in different ways. The first one is calculated from actual contribution, the second one from its complement. In practical applications, calculated values are the same or 
very close to each other. (This would remain to be the case as long as all the basic events included in minimal cutsets have small probabilities.)

Finally, the RIF (which corresponds to the RAW) is calculated as:

$$
I_{i}^{I}=\frac{Q_{T O P}\left(Q_{i}=1\right)}{Q_{T O P}}
$$

where the term $Q_{T O P}\left(Q_{i}=1\right)$ refers to the top event probability recalculated with probability $Q_{i}$ set to 1 .

"Sensitivity calculations" for basic event " $i$ " are done by dividing and multiplying the probability $Q_{i}$ by a sensitivity factor and recalculating the corresponding top event probabilities. (Sensitivity factor can have any value larger than 1 , with default set to 10.) Calculated, also, is the "sensitivity value" defined as:

$$
S_{i}=\frac{Q_{T O P, U, i}}{Q_{T O P, L, i}}
$$

where $Q_{T O P, U, i}$ and $Q_{T O P, L, i}$ refer to top event probability recalculated with $Q_{i}$ multiplied and divided by the sensitivity factor, respectively. Reported are all three values, $S_{i}, Q_{T O P, U, i}$ and $Q_{T O P, L, i+}$ It is noted that these "sensitivity calculations" can be related to the reliability importance discussed in this monograph as:

$$
I_{r e l}=\frac{Q_{T O P, U}-Q_{T O P, L}}{Q_{i}\left(\sigma-\frac{1}{\sigma}\right)}
$$

where $\sigma$ refers to the "sensitivity factor".

The importance measures, $I_{i}^{R}, I_{i}^{F}$ and $I_{i}^{I}$ can be calculated for different basic event groups in the same way as for individual basic events, where the probabilities are set to zero or to 1 for all basic events which belong to the group.

The importance measures, $I_{i}^{R}, I_{i}^{F}$ and $I_{i}^{I}$ are also calculated for the parameters which are used to calculate basic events probabilities: probabilities of failure on demand, failure rates, frequencies, repair rates (mean times to repair), test intervals and mission times. The formulas correspond to the above: 


$$
I_{i}^{R}=\frac{Q_{T O P}}{Q_{T O P, B E S T}} \quad \text { and } \quad I_{i}^{I}=\frac{Q_{T O P, W O R S T}}{Q_{T O P}} .
$$

The term $Q_{T O P, B E S T}$ refers to the top event probability recalculated with parameter set to the "best theoretically possible" value (zero for all parameters). The term $Q_{\text {TOP,WORST }}$ refers to the top event probability recalculated with parameter set to the "worst theoretically possible" value ( 1 for probability of failure on demand, the infinity for all others). The $I_{i}^{F}$ is calculated from $I_{i}^{R}$ in the same manner as above. 


\section{Appendix B. Demonstrating examples based on a simplified PRA model}

\section{B.1 Description of simplified PRA model used for demonstration}

The PRA model which was used for calculations of risk importance measures in this monograph was a very simple PRA model based on Pressurized Water Reactor. This model was developed merely for exercising purposes and its results cannot be used as any indications of real risk measures. It was built on RiskSpectrum ${ }^{\circledR}$ PSA, Version 1.2.0.7.

The model has eight event trees, which are for the initiators:

1. Large LOCA

2. Small LOCA

3. Steam Generator Tube Rupture

4. Plant Transient

5. Main Steam Line Break

6. Loss of Offsite Power

7. Loss of Essential Service Water

8. Loss of Component Cooling Water.

To illustrate the level of details, Figure B-1 shows the event tree for Small LOCA.

Meaning of the event tree headers in Figure B-1 is as follows:

SLOCA Initiating event - Small break LOCA

RXTRIP Reactor trip

HPSI High Pressure Safety Injection

EFW Emergency Feedwater provides secondary heat sink

RCD_SLO Cooldown and depressurization of RCS to achieve LPSI parameters. Function covers operator's actions and relief valves (Pressurizer and SG PORVs)

LPSI Low Pressure Safety Injection

LPREC Low Pressure Recirculation following the depletion of RWST 
106 Risk Importance Measures in the Design and Operation of NPPs

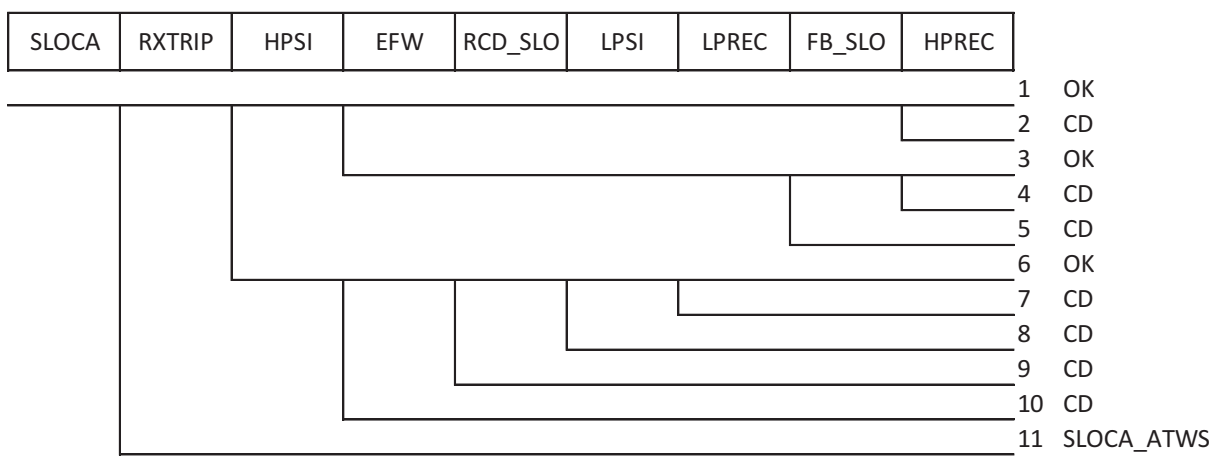

Figure B-1 Small LOCA event tree.

FB_SLO Primary feed and bleed. Function covers operator's actions and relief valves (Pressurizer PORVs)

HPREC Switchover to HP recirculation following the depletion of RWST

The model includes simplified fault trees for the most important plant systems:

+ High Pressure Safety Injection;

+ Low Pressure Safety Injection;

+ Emergency Feedwater;

+ Charging Pumps (CVCS)

+ Component Cooling Water;

+ Essential Service Water;

+ Pressurizer PORVs (for performing Primary Feed and Bleed function);

+ Main Steam Valves;

- Power distribution system including Emergency Diesel Generators.

Power distribution fault tree model is limited to explicit model of AC power for main components such as pumps and motor-operated valves. It is very simple and consists of train-level basic events for distribution (Distribution Train A and Train B) and component-level basic events for Emergency Diesel Generators and their circuit breakers. Instrument power, DC power and Engeneered Safety Features Actuation System and other supporting systems (e.g. Instrument Air) are not represented in the fault trees explicitly. 


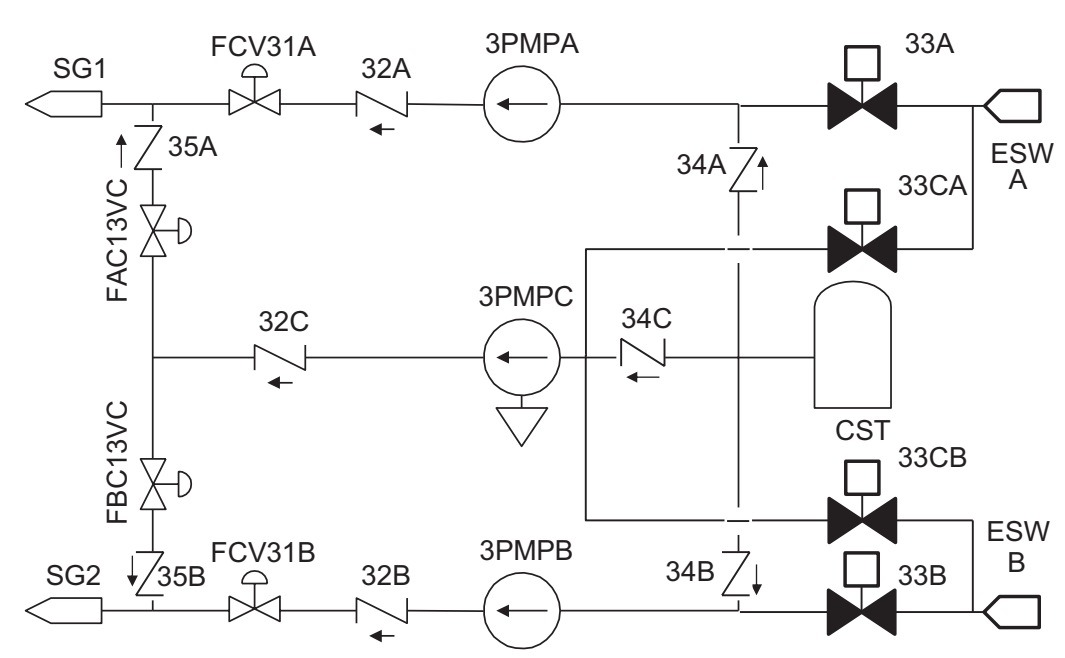

Figure B-2 Emergency feedwater system as modeled.

To illustrate modeling level of detail, Figure B-2 is provided which shows simplified flow diagram of Emergency Feedwater system. The components shown in the figure are explicitly presented in the fault tree model by basic events.

The parameters used to quantify the model are considered to reflect the typical values which are currently used for particular types of equipment and initiating events. For example, failure rates and failure probabilities assigned to equipment are comparable to the values from NUREG/CR-6928 and initiating event frequencies are comparable to those provided in NUREG/CR-5750.

With cutoff set at $1 \mathrm{E}-12$, the model generates 11231 minimal cutsets and calculates, by "mincut upper bound" approximation, the CDF at $4.0448 \mathrm{E}-05 / \mathrm{yr}$. With cutoff set at 1E-20, the model generates 69575 minimal cutsets and calculates, by the same approximation, the CDF at 4.0452E-05/yr.

\section{B.2 Examples concerning theoretical relation between RAW and RRW and importance measures for single basic event (Examples B1 through B4)}

Introduction to Examples B1 through B4

In this section we address, through a set of four examples, some specific considerations from Sections 5.1 through 5.6. The demonstrations are based on the RiskSpectrum ${ }^{\circledR}$ PRA model described in B.1. This model 
provides the list of importance measures for 212 basic events (excluding the initiating events) at minimal cutsets cutoff of $1 \mathrm{E}-12$. Table B-1 lists the results for the top 20 of them when sorted by RRW in decreasing order, in columns under the entry "Values Taken from RS Model". The first column, $P(A)$, contains basic event probabilities in the RiskSpectrum ${ }^{\circledR}$ model. The second column, $I_{f c}$, contains the F-V importance measures reported by RiskSpectrum ${ }^{\circledR}$. (The ways how RiskSpectrum ${ }^{\circledR}$ calculates particular importance measures are described in Appendix A. It should be noted that RiskSpectrum ${ }^{\circledR}$ also calculates the "fractional contribution". However, the value of FV calculated by RiskSpectrum ${ }^{\circledR}$ more closely corresponds to the $I_{f c}$ as used in this monograph. This is because RiskSpectrum ${ }^{\circledR}$ calculates the FV from actual contribution, while the "fractional contribution" is in RiskSpectrum ${ }^{\circledR}$ calculated from the complement. Therefore, in the monograph, when we compare calculated $I_{f_{c}}$ values against RiskSpectrum ${ }^{\circledR}$, we use the corresponding F-V value from RiskSpectrum ${ }^{\circledR}$ as a reference. It is, however pointed that in practical applications one should not expect any relevant difference between the two such that it would affect the conclusions.) The third and fourth column under "Values Taken from RS Model", $I_{R R W}$ and $I_{R A W}$, contain the RDF and RIF measures reported by RiskSpectrum ${ }^{\circledR}$, respectively. The fifth and the sixth column under the same entry show the results of the sensitivity calculations (which are further discussed in Example B4 below).

Table B-2 contains the calculations, for the same top 20 basic events which are related to the considerations in the Sections 5.1 through 5.6. These results are further discussed in the Examples B1 through B4 below, with purpose to demonstrate some specific points from the mentioned sections.

Example B1: Theoretical relation between $R A W$ and $R R W$

The first example is meant to illustrate the theoretical relation between RAW and RRW. Table B-2 contains, under the entry "Example B1", the $I_{R R W}$ values (designated as $I_{R R W}\left(I_{R A W}\right)$ ) which were calculated by the formula (5-9) from the values of $I_{R A W}$ and $P(A)$ reported by RiskSpectrum ${ }^{\circledR}$ and presented in Table B-1. It can be seen that these calculated values closely follow the reported $I_{R R W}$ values (RS's RDFs), demonstrating the theoretical connection. It needs to be mentioned that, on the other hand, calculations of $I_{R A W}$ from reported $I_{R R W}$ 


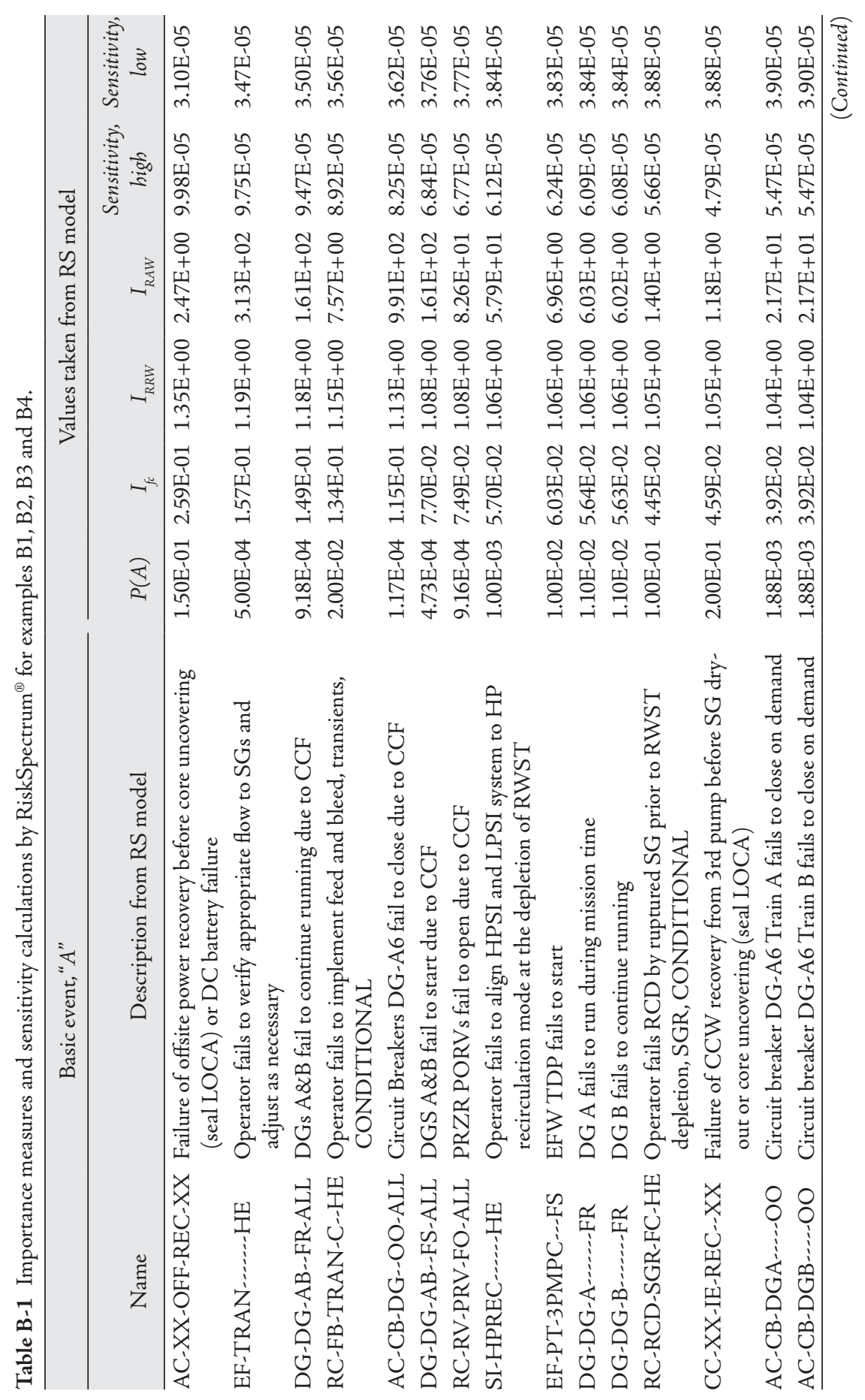




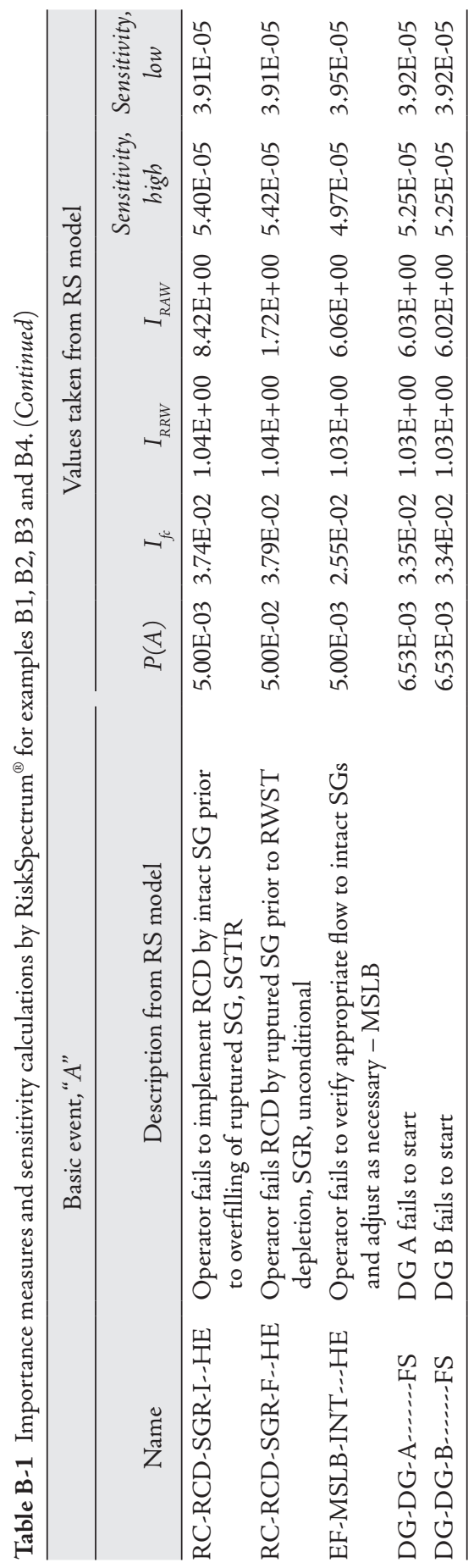




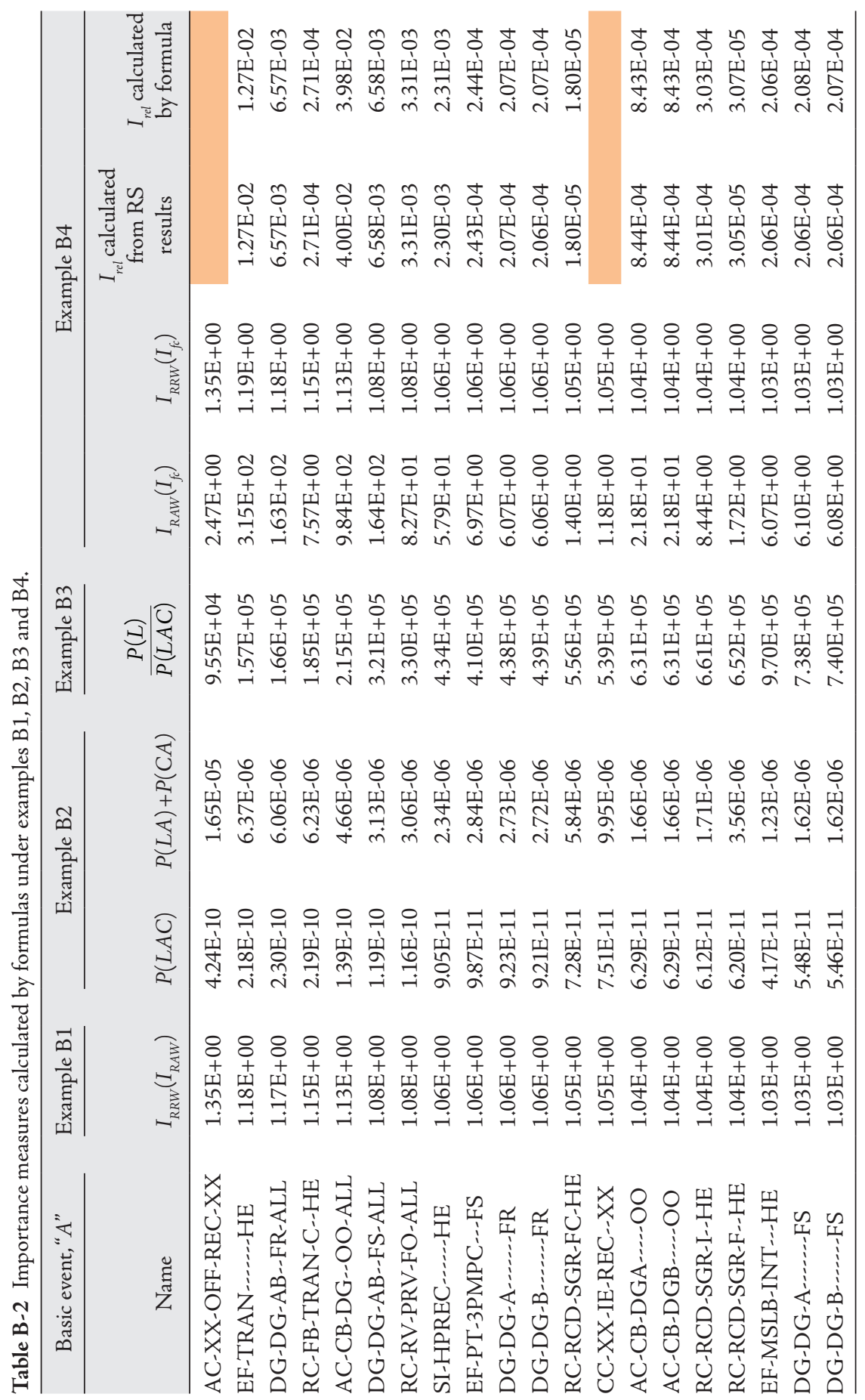


(i.e. RDF) by (5-9) did not present good demonstration as there were many significant deviations from the reported $I_{R A W}(i, e$. RIF) values (not shown in Table B-2). However, there is obvious reason behind. Calculation of $I_{R A W}$ by $(5-9)$ depends on the term $\frac{1}{I_{R R W}}$, where $I_{R R W}$ is in most of the cases (basically, all except the top 10 or so in Table B-1) only slightly higher than 1.0 . More precise calculation of $I_{R A W}$ by $(5-9)$ would require number of decimal places for RDF much larger than two decimal places which are actually reported by RiskSpectrum ${ }^{\circledR}$.

Example B2: Demonstrating $P(L A C)<<P(L A)+P(C A)$

Quantification tools used in NPP PRAs often use the "Mincut Upper Bound" (MCUB) approximation for the top event probability calculations which assumes that all minimal cutsets in the list are mutually independent (which is, generally, not the case). For the purpose of this example we will accept this assumption (i.e. that minimal cutsets can be considered as mutually independent for top event quantification purposes) without trying to further evaluate its potential implications. (Just as an illustration: RiskSpectrum ${ }^{\circledR}$ can quantify the top event by MCUB and by the $1^{\text {st }}, 2^{\text {nd }}$ and $3^{\text {rd }}$ order approximation of inclusion-exclusion principle [39]. The actual result for the given list of minimal cutsets (from Section B.1) lies between the $2^{\text {nd }}$ and $3^{\text {rd }}$ order approximation. The MCUB quantification of the RiskSpectrum ${ }^{\circledR}$ model described in B.1 at $1 \mathrm{E}-12$ cutoff gives the result of $4.0448 \mathrm{E}-05$. The $2^{\text {nd }}$ order approximation gives $4.0212 \mathrm{E}-05$ and the $3^{\text {rd }}$ order gives $4.0214 \mathrm{E}-05$. This indicates that the difference between the MCUB and actual result is not larger than a fraction of percent.)

Table B-2 shows calculated both terms $P(L A C)$ and $P(L A)+P(C A)$ for the top 20 basic events (when sorted by RDF values reported by RiskSpectrum ${ }^{\circledR}$ ) from Table B-1 (two columns under the entry "Example B2"). The above stated assumption makes the terms $L$ and $A C$ mutually independent and, hence: $P(L A C) \approx P(L) P(A C)$. Other terms of interest were estimated as:

$+P(L)=\frac{P(B)}{I_{R R W}}$, where $P(B)$ refers to the CDF calculated by RiskSpectrum ${ }^{\circledR}(4.0448 \mathrm{E}-05)$ and $I_{R R W}$ refers to RRW reported by RiskSpectrum ${ }^{\circledR}$; 
+ $P(A C)=I_{f_{c}} P(B)$, where $I_{f_{c}}$ refers to $\mathrm{FV}$ reported by RiskSpectrum ${ }^{\circledR}$;

+ $P(L A)=P(L) P(A)$, where $\mathrm{P}(\mathrm{A})$ refers to the basic event probability in the RS model.

As it can be seen in all cases in Table B-2, the value of the term $P(L A)+$ $P(A C)$ is by orders of magnitude larger than of the term $P(L A C)$. Actually, the largest observed ratio $\frac{P(L A C)}{P(L A)+P(C A)}$ among all 212 basic events in the RS importance list was 3.86E-05, which clearly demonstrates the point.

Example B3: Demonstrating $P(B)-P(C A)>>P(R A C)$

The actual theoretical expression for $P(R)$ is: $P(R)=P(B)-P(C A)+$ $P(R A C)$. Thus, the above statement can be reformulated to: $P(R)>>2$ $P(R A C)$ or : $\frac{P(R)}{P(R A C)}>>2$. Both terms, $P(R)$ and $P(R A C)$, were estimated under Example B2 above. The ratio is for the same top 20 basic events (from Table B-1) shown in Table B-2 under the entry "Example B3". It can be seen that calculated values are, indeed, by orders of magnitude higher than 2. In fact, the smallest observed ratio among all 212 basic events in the RiskSpectrum ${ }^{\circledR}$ importance list was $9.55 \mathrm{E}+04$, which clearly demonstrates the point.

Example B4: Use of formulas derived in Sections 5.3, 5.4 and 5.5

to calculate the importance measures for single basic event

Table B-2 shows, in the column $I_{R A W}\left(I_{f_{c}}\right)$ under the entry "Example B4", RAW values for the 20 basic events from Table B-1 which were calculated from the corresponding $I_{f c}$ reported by the RiskSpectrum ${ }^{\circledR}$ (and shown in the same Table B-1) by means of the expression (5-17). The same table contains, in the column $I_{R R W}\left(I_{f c}\right)$ under the entry "Example B4", RRW values for the same basic events, calculated on the same basis by the formula (5-18). As can be seen, the values closely follow the corresponding values provided in Table B-1 which were directly reported by the RiskSpectrum ${ }^{\circledR}$. The differences are attributable to the rounding or truncation errors or approximations used by RiskSpectrum ${ }^{\circledR}$ in quantifying the top event. 
The columns "Sensitivity, High" and "Sensitivity, Low" in Table B-1 contain the top event probability $P(B)$ obtained with basic event probability $P(A)$ being multiplied and divided, respectively, by the sensitivity factor of 10 . (The nominal value of $P(B)$ is CDF of $4.0448 \mathrm{E}-05(/ \mathrm{yr})$ quantified at cutoff of $1 \mathrm{E}-12$, as described in B.1.) The "Sensitivity, High" and "Sensitivity, Low" values represent the terms $Q_{T O P, U}$ and $Q_{T O P, L}$, respectively, from Appendix A. Considering that $\sigma$ is sensitivity factor of 10 and that $Q_{i}=P(A)$ (referring to the formula for $I_{r e l}$ in Appendix A), the reliability importance for the 20 basic events considered can be approximated by the formula:

$$
I_{\text {rel }}=\frac{Q_{T O P, U}-Q_{T O P, L}}{9.9 P(A)}
$$

The results of this approximation are shown in the column " $I_{\text {rel }}$ calculated from RS results" under entry "Example B4" in Table B-2. These results represent the reliability importance measure for particular basic events as calculated from the RiskSpectrum ${ }^{\circledR}$ results. On the other hand, the column " $I_{\text {rel }}$ calculated by formula" under the same entry in Table B-2 shows the corresponding reliability importance calculated by the formula (5-22). As can be seen, all the results shown are very close to each other. Table B-2 does not display the results for the two cases. In these cases the basic event probability $P(A)$ is larger than 0.1 and multiplying it by the factor of 10 results with a value (probability) larger than 1 . As in such case RiskSpectrum ${ }^{\circledR}$ would truncate the $P(A)$ value to 1 , the result is not directly comparable to the result obtained by the formula.

\section{B.3 Examples of calculation of component-level importance measures}

Introduction to Examples B5 through B8

In this section of the appendix, details of the calculation of component-level importance measures are demonstrated, along with a validation of importance measures calculated using the formulas presented in this monograph.

Importance measures are calculated in two different ways: (a) using the referential PRA model through modification and requantification, referred to as "full PRA model requantification", and (b) using the formulas developed in this monograph. Comparable results obtained in both the cases showed the validation of the formulas developed in this monograph. 
Details of the calculations are presented for the following components, selected in order to cover and illustrate different cases discussed in the monograph:

+ Component represented by multiple basic events, none involved in a CCF group (FT module):

+ CVCS PDP, Example B5 (Table B-3),

+ Component represented by multiple different FT modules or basic events in different accident scenarios, none involved in a CCF group:

+ CVCS PDP with two different operating failure rates, Example B6 (Table B-4),

+ Single basic event (failure mode) involved in a CCF group of size $m=2$ :

+ EFW MDP 1 fails to start, Example B7.1 (Table B-5),

+ EFW MDP 1 fails to run for a mission time, Example B7.2 (Table B-6),

+ EDG 1 fails to start, Example B7.3 (Table B-7),

+ EDG 1 fails to run for a mission time, Example B7.4 (Table B-8),

+ Single basic event (failure mode) involved in a CCF group of size $m=3$ :

+ EFW Discharge Check Valve failure to open, Example B7.5 (Table B-9)

+ Component represented by multiple basic events (failure modes) involved in CCF groups

+ EFW MDP 1, Example B8.1 (Table B-10),

+ EDG 1, Example B8.2 (Table B-11).

One way to calculate the importance measures, achievable in practice, is to appropriately modify the PRA model structure (to reflect failed or "perfectly reliable" status of considered component) and then to regenerate the minimal cutsets to calculate conditional top event probabilities. These conditional top event probabilities are then used along with nominal top event probability to calculate the considered importance measure. This way of calculation of an importance measure is, as mentioned, referred to as the "full PRA model requantification" (and is further discusse in the text below). Unfortunately, this may not be the most 


\section{Risk Importance Measures in the Design and Operation of NPPs}

\section{Table B-3 Example B5: CVCS PDP.}

\begin{tabular}{|c|c|}
\hline \multicolumn{2}{|l|}{ Part 1: Information from PRA Model and Its Results } \\
\hline Probability, $P\left(A_{j}\right)$ & Fractional Contribution, $I_{f c, j}$ \\
\hline $2.00 \mathrm{E}-03$ & $9.05 \mathrm{E}-03$ \\
\hline $1.20 \mathrm{E}-04$ & $5.43 \mathrm{E}-04$ \\
\hline $2.00 \mathrm{E}-03$ & $9.05 \mathrm{E}-03$ \\
\hline \multicolumn{2}{|l|}{ Expression (6-9): $P\left(A^{*}\right) \approx P\left(A_{F S}\right)+P\left(A_{F R}\right)+P\left(A_{T M}\right)=4.12 \mathrm{E}-03$} \\
\hline $\begin{array}{l}\text { Part 2: Direct Calculations by Conditional Top Event } \\
\text { Probabilities (Full RiskSpectrum }{ }^{\circledR} \text { PRA Model } \\
\text { Requantification) }\end{array}$ & $\begin{array}{l}\text { Part 3: Calculations by Formulas Using the } \\
\text { Information from PRA Model and Its Results }\end{array}$ \\
\hline \multicolumn{2}{|c|}{ Fractional Contribution, $I_{f c}^{*}$} \\
\hline $\begin{array}{l}\text { For the FT module expressed by (6-7), } A^{*}=0 \text { implies } \\
A_{i}=0 \text { for every } i, i=1, \ldots, n \text {. Set all three basic events, } \\
A_{F S}, A_{F R} \text { and } A_{T M} \text { to "false" state ans rerun: } \\
P\left(B \mid \bar{A}^{*}\right)=3.97 \mathrm{E}-05 \\
\text { Expression (B-1): } I_{f c}^{*} \approx 1-\frac{P\left(B \mid \bar{A}^{*}\right)}{P(B)}=\mathbf{1 . 8 6 E - 0 2}\end{array}$ & $\begin{array}{l}\text { Expression (6-11): } I_{f c}^{*}=I_{f c, X} \frac{P\left(A^{*}\right)}{P\left(A_{X}\right)}=\mathbf{1 . 8 6 \mathrm { E } - 0 2} \\
\text { (Note: } A_{X} \text { is any of the three basic events, } A_{F S}, A_{F R} \\
\text { or } A_{T M} \cdot \text { ) } \\
\text { Expression (6-12): } I_{f c}^{*}=I_{f c, F S}+I_{f c, F R}+I_{f c, T M}=\mathbf{1 . 8 6 \mathrm { E } - 0 2}\end{array}$ \\
\hline \multicolumn{2}{|c|}{ Risk Achievement Worth, $I_{R A W}^{*}$} \\
\hline $\begin{array}{l}\text { For the FT module expressed by (6-7), } A^{*}=1 \text { implies } \\
A_{i}=1 \text { for at least one } i, i=1, \ldots, n \text {. Set any of the three } \\
\text { basic events, } A_{F S}, A_{F R} \text { or } A_{T M} \text { to "true" state and } \\
\text { rerun: } \\
P\left(B \mid A^{*}\right)=2.20 \mathrm{E}-04 \\
I_{R A W}^{*}=\frac{P\left(B \mid A^{*}\right)}{P(B)}=5.43 \mathrm{E}+00\end{array}$ & $\begin{array}{l}\text { Expression (6-4): } \\
I_{R A W}^{*}=1+I_{f c}^{*}\left(\frac{1}{P\left(A^{*}\right)}-1\right)=5.51 \mathrm{E}+00\end{array}$ \\
\hline \multicolumn{2}{|c|}{ Risk Reduction Worth, $I_{R R W}^{*}$} \\
\hline$I_{R R W}^{*}=\frac{P(B)}{P\left(B \mid \bar{A}^{*}\right)}=1.02 \mathrm{E}+00$ & $\begin{array}{l}\text { Expression (6-5): } I_{R R W}^{*}=\frac{1}{1-I_{f c}^{*}}=1.02 \mathrm{E}+00 \\
\text { Theoretical relation to } I_{R A W}^{*},(5-6): \\
I_{R R W}=\frac{1-P\left(A^{*}\right)}{1-P\left(A^{*}\right) I_{R A W}^{*}}=1.02 \mathrm{E}+00\end{array}$ \\
\hline \multicolumn{2}{|c|}{ Reliability Importance, $I_{r e l}^{*}$} \\
\hline $\begin{array}{l}\text { Multiply all three basic event probabilities, } P\left(A_{F S}\right) \text {, } \\
P\left(A_{F R}\right) \text { and } P\left(A_{T M}\right) \text {, by sensitivity factor } \sigma=10 \text { and } \\
\text { rerun: } \\
P_{U}(B)=4.71 \mathrm{E}-05 \\
\text { Expression }(\mathrm{B}-2): I_{r e l}^{*} \approx \frac{P_{U}(B)-P(B)}{(\sigma-1) P\left(A^{*}\right)}=\mathbf{1 . 8 1 E - 0 4}\end{array}$ & $\begin{array}{l}\text { Expression }(6-6) \text { : } \\
I_{r e l}^{*}=P(B) \frac{I_{f c}^{*}}{P\left(A^{*}\right)}=1.83 \mathrm{E}-04\end{array}$ \\
\hline
\end{tabular}


Table B-4: Example B6: CVCS PDP with two different operating failure rates.

\begin{tabular}{|c|c|c|c|c|}
\hline \multicolumn{5}{|c|}{ Part 1: Information from PRA Model and Its Results } \\
\hline \multirow{2}{*}{\multicolumn{3}{|c|}{$\begin{array}{l}\text { FT Module 1: Sequences Other Than Loss of } \\
\text { ESW/CCW }\end{array}$}} & \multicolumn{2}{|c|}{ FT Module 2: Sequences with Loss of ESW/CCW } \\
\hline & & & \multicolumn{2}{|c|}{ Bas. Ev., $\left(A_{j 2}\right) \quad$ Prob., $P\left(A_{j 2}\right) \quad$ FC, $I_{f c, j 2}$} \\
\hline \multicolumn{3}{|c|}{ Bas. Ev., $\left(A_{j 1}\right) \quad$ Prob., $P\left(A_{j 1}\right) \quad \mathrm{FC}, I_{f c, j 1}$} & \multirow{3}{*}{$\begin{array}{c}A_{F S} \\
A_{F R, L} \\
A_{T M} \\
\text { Expr. (7-16): }\end{array}$} & $2.00 \mathrm{E}-03 \quad 9.05 \mathrm{E}-03$ \\
\hline & $2.00 \mathrm{E}-03$ & & & \\
\hline \multicolumn{3}{|c|}{$\begin{array}{lll}A_{F R} & 1.20 \mathrm{E}-04 & 5.43 \mathrm{E}-04 \\
A_{T M} & 2.00 \mathrm{E}-03 & 9.05 \mathrm{E}-03\end{array}$} & & $9.05 \mathrm{E}-03$ \\
\hline \multicolumn{3}{|c|}{$\begin{array}{l}\text { Expr. (7-16): } \\
P\left(A_{1}^{*}\right) \approx P\left(A_{F S}\right)+P\left(A_{F R}\right)+P\left(A_{T M}\right)=4.12 \mathrm{E}-03\end{array}$} & \multicolumn{2}{|c|}{$\begin{array}{l}\text { Expr. (7-16): } \\
P\left(A_{2}^{*}\right) \approx P\left(A_{F S}\right)+P\left(A_{F R, L}\right)+P\left(A_{T M}\right)=2.77 \mathrm{E}-02\end{array}$} \\
\hline \multicolumn{3}{|c|}{$\begin{array}{l}\text { Part 2: Direct Calculations by Conditional Top Event } \\
\text { Probabilities (Full RiskSpectrum }{ }^{\circledast} \text { PRA Model } \\
\text { Requantification) }\end{array}$} & \multicolumn{2}{|c|}{$\begin{array}{l}\text { Part 3: Calculations by Formulas Using the } \\
\text { Information from PRA Model and Its Results }\end{array}$} \\
\hline \multicolumn{5}{|c|}{ Fractional Contribution, $I_{f c}^{*}$} \\
\hline \multicolumn{3}{|c|}{$\begin{array}{l}\text { Set all four basic events, } A_{F S}, A_{F R}, A_{F R, L} \text { and } A_{T M} \text { to } \\
\text { "false" state and rerun: } \\
P\left(B \mid \bar{A}_{1}^{*} \bar{A}_{2}^{*}\right)=3.97 \mathrm{E}-05 \\
\text { Expression (B-1): } I_{f c}^{*} \approx 1-\frac{P\left(B \mid \bar{A}_{1}^{*} \bar{A}_{2}^{*}\right)}{P(B)}=9.04 \mathrm{E}-02\end{array}$} & \multicolumn{2}{|c|}{$\begin{array}{l}\text { Expressions (7-7) and (7-19): } \\
I_{f c, L}^{*}=\frac{I_{f c, F R}}{P\left(A_{F R}\right)} P\left(A_{1}^{*}\right)=4.43 \mathrm{E}-03 \\
I_{f c, 2}^{*}=\frac{I_{f c, F R, L}}{P\left(A_{F R, L}\right)} P\left(A_{2}^{*}\right)=8.59 \mathrm{E}-02 \\
I_{f c}^{*}=I_{f c, l}^{*}+I_{f c, 2}^{*}=9.03 \mathrm{E}-02\end{array}$} \\
\hline \multicolumn{5}{|c|}{ Risk Achievement Worth, $I_{R A W}^{*}$} \\
\hline \multicolumn{3}{|c|}{$\begin{array}{l}\text { At least one basic event in each module needs to be } \\
\text { set to "true" state. Set basic events } A_{F R} \text { and } A_{F R, L} \text { to } \\
\text { "true" state and rerun: } \\
P\left(B \mid A_{1}^{*} A_{2}^{*}\right)=2.20 \mathrm{E}-04 \\
I_{R A W}^{*}=\frac{P\left(B \mid A_{1}^{*} A_{2}^{*}\right)}{P(B)}=\mathbf{5 . 0 4 \mathrm { E } + 0 0}\end{array}$} & \multicolumn{2}{|c|}{$\begin{array}{l}\text { Expression (7-14): } \\
\frac{I_{f c, 1}^{*}}{P\left(A_{1}^{*}\right)}=1.08 \mathrm{E}+00 ; \frac{I_{f c, 2}^{*}}{P\left(A_{2}^{*}\right)}=3.10 \mathrm{E}+00 \\
I_{R A W}^{*}=I+\sum_{i=l}^{n} \frac{I_{f c, i}^{*}}{P\left(A_{i}^{*}\right)}-I_{f c}^{*}=\mathbf{5 . 0 9} \mathbf{E}+00\end{array}$} \\
\hline
\end{tabular}

(Continued)

practical approach for at least two reasons: 1 ) number of components may be very large, and 2) regeneration of MCSs may take long time and considerable effort (depending on a PRA tool).

Under such conditions being able to calculate component-level importance measures from the existing results (reported importance measures for basic events) of base case PRA model quantification may be helpful. Formulas from Sections 6 through 9 rely on such readily available results (although, in some instances the insider's knowledge on the PRA model may be needed in order to recognize which "results" are required). The purpose of this set of examples is to compare the importance measures 
Table B-4: Example B6: CVCS PDP with two different operating failure rates. (Continued)

\begin{tabular}{|c|c|}
\hline $\begin{array}{l}\text { Part 2: Direct Calculations by Conditional Top Event } \\
\text { Probabilities (Full RiskSpectrum }{ }^{\otimes} \text { PRA Model } \\
\text { Requantification) }\end{array}$ & $\begin{array}{l}\text { Part 3: Calculations by Formulas Using the } \\
\text { Information from PRA Model and Its Results }\end{array}$ \\
\hline \multicolumn{2}{|c|}{ Risk Reduction Worth, $I_{R R W}^{*}$} \\
\hline$I_{R R W}^{*}=\frac{P(B)}{P\left(B \mid \bar{A}_{1}^{*} \bar{A}_{2}^{*}\right)}=1.10 \mathrm{E}+00$ & $\begin{array}{l}\text { Expression (6-5): } I_{R R W}^{*}=\frac{1}{1-I_{f c}^{*}}=1.10 \mathrm{E}+00 \\
\text { To calculate RRW from the theoretical relation to } \\
I_{R A W}^{*},(5-6 a) \text {, component-level failure probability } \\
P\left(A^{*}\right) \text { needs to be calculated first. From }(7-12): \\
P\left(X_{1}\right)=\frac{\frac{I_{f c, 1}^{*}}{P\left(A_{1}^{*}\right)}}{\frac{I_{f c, 1}^{*}}{P\left(A_{1}^{*}\right)}+\frac{I_{f c, 2}^{*}}{P\left(A_{2}^{*}\right)}} P\left(X_{2}\right)=\frac{\frac{I_{f c, 2}^{*}}{P\left(A_{2}^{*}\right)}}{\frac{I_{f c, 1}^{*}+\frac{I_{f c, 2}^{*}}{P\left(A_{1}^{*}\right)}}{P\left(A_{2}^{*}\right)}} \\
\text { Thus: } P\left(X_{1}\right)=2.57 \mathrm{E}-01 ; P\left(X_{2}\right)=7.43 \mathrm{E}-01, \\
\text { and, from (7-9): } P\left(A^{*}\right)=\sum_{i=1}^{n} P\left(X_{i}\right) P\left(A_{i}^{*}\right)=2.16 \mathrm{E}-02 . \\
\text { Finally, from (5-6): } I_{R R W}=\frac{1-P\left(A^{*}\right)}{1-P\left(A^{*}\right) I_{R A W}^{*}}=1.10 \mathrm{E}+00\end{array}$ \\
\hline Reliability I & ortance, $I_{r e l}^{*}$ \\
\hline 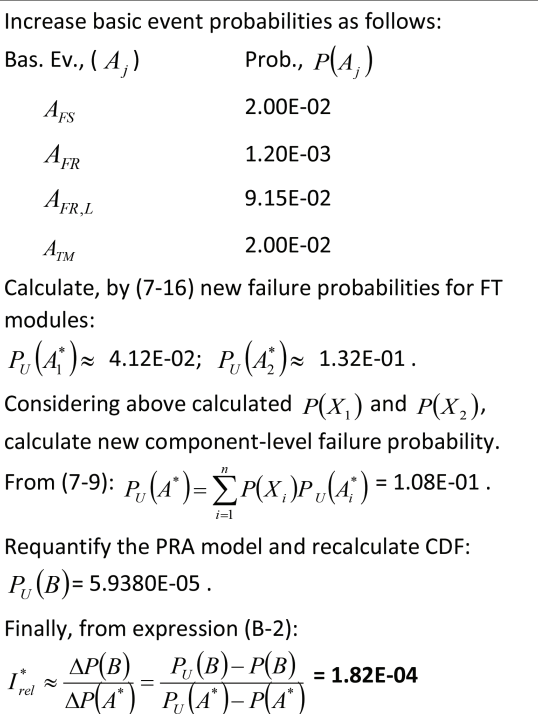 & $\begin{array}{l}\text { Expression }(6-6): \\
I_{r e l}^{*} \approx P(B) \frac{I_{f c}^{*}}{P\left(A^{*}\right)}=1.82 \mathrm{E}-04\end{array}$ \\
\hline
\end{tabular}


Table B-5 Example B7.1: EFW MDP1 fails to start.

\begin{tabular}{|c|c|}
\hline \multicolumn{2}{|l|}{ Part 1: Information from PRA Model and Its Results } \\
\hline \multicolumn{2}{|l|}{ CCF Model Parameters: $Q_{t}=P(A)=2.00 \mathrm{E}-03 ; \alpha_{2}=1.10 \mathrm{E}-01$} \\
\hline Basic Event, $V_{k} \quad$ Probability, $P\left(V_{k}\right)$ & RAW, $I_{R A W, k}$ \\
\hline$V_{1}$ (MDP 1 fails) & $1.07 \mathrm{E}+00$ \\
\hline $\begin{array}{l}V_{2} \text { (MDPs } 1 \text { and } 2 \text { fail) } \\
\text { (Note: Probabilities } P\left(V_{k}\right) \text { were calculated by Riskspect }\end{array}$ & 8.57E-04 \\
\hline $\begin{array}{l}\text { Part 2: Direct Calculations by Conditional Top Event } \\
\text { Probabilities (Full RiskSpectrum }{ }^{\circledR} \text { PRA Model } \\
\text { Requantification) }\end{array}$ & $\begin{array}{l}\text { Part 3: Calculations by Formulas Using the } \\
\text { Information from PRA Model and Its Results }\end{array}$ \\
\hline \multicolumn{2}{|c|}{ Fractional Contribution, $I_{f c}$ (2. Failure or Unavailability with CCF Potential) } \\
\hline $\begin{array}{l}A=0 \text { implies } V_{k}=0 \text { for every } k, k=1, \ldots, M \text {. Set both } \\
\text { CCF basic events, } V_{1} \text { and } V_{2} \text { to "false" state and rerun: } \\
P(B \mid \bar{A})=4.0409 \mathrm{E}-05 \\
\text { Expression (B-1): } I_{f c} \approx 1-\frac{P(B \mid \bar{A})}{P(B)}=\mathbf{9 . 6 4 \mathrm { E } - 0 4}\end{array}$ & Expression (8-8): $I_{f c}=\sum_{k=1}^{M} I_{f c, k}=9.64 \mathrm{E}-04$ \\
\hline \multicolumn{2}{|c|}{ Risk Achievement Worth, $I_{R A W}$ (2. Failure or Unavailability with CCF Potential) } \\
\hline $\begin{array}{l}\text { Modify nominal probabilities } P\left(V_{k}\right) \text { to conditional } \\
\frac{P\left(V_{k}\right)}{P(A)} \text { for both basic events } V_{1} \text { and } V_{2} \text { and rerun: } \\
P(B \mid A)=6.0122 \mathrm{E}-05 \\
I_{R A W}=\frac{P(B \mid A)}{P(B)}=1.49 \mathrm{E}+00\end{array}$ & $\begin{array}{l}\text { Expression (8-9): } \\
I_{R A W}=1+I_{f c}\left(\frac{1}{P(A)}-1\right)=\mathbf{1 . 4 8 \mathrm { E } + 0 0}\end{array}$ \\
\hline \multicolumn{2}{|c|}{ Risk Reduction Worth, $I_{R R W}$ (2. Failure or Unavailability with CCF Potential) } \\
\hline$I_{R R W}=\frac{P(B)}{P(B \mid \bar{A})} \approx 1.00 \mathrm{E}+00$ & $\begin{array}{l}\text { Expression (8-11): } I_{R R W}=\frac{1}{1-I_{f c}} \approx 1.00 \mathrm{E}+00 \\
\text { Theoretical relation to } I_{R A W},(5-9): \\
I_{R R W}=\frac{1-P(A)}{1-P(A) I_{R A W}} \approx 1.00 \mathrm{E}+00\end{array}$ \\
\hline \multicolumn{2}{|c|}{ Reliability Importance, $I_{r e l}$ (2. Failure or Unavailability with CCF Potential) } \\
\hline $\begin{array}{l}\text { Multiply both basic event probabilities, } P\left(V_{1}\right) \text { and } \\
P\left(V_{2}\right) \text {, by sensitivity factor } \sigma=10 \text { and rerun: } \\
P_{U}(B)=4.0802 \mathrm{E}-05 \\
\text { Expression }(\mathrm{B}-2): I_{r e l} \approx \frac{P_{U}(B)-P(B)}{(\sigma-1) P(A)}=\mathbf{1 . 9 7 E - 0 5}\end{array}$ & $\begin{array}{l}\text { Expression (8-12): } \\
I_{r e l}=P(B) \frac{I_{f c}}{P(A)}=1.95 \mathrm{E}-05\end{array}$ \\
\hline
\end{tabular}




\section{Risk Importance Measures in the Design and Operation of NPPs}

\section{Table B-6 Example B7.2: EFW MDP1 fails to run for a mission}

time.

\begin{tabular}{|c|c|}
\hline \multicolumn{2}{|l|}{ Part 1: Information from PRA Model and Its Results } \\
\hline \multicolumn{2}{|l|}{ CCF Model Parameters: $Q_{t}=P(A)=1.20 \mathrm{E}-04 ; \alpha_{2}=2.00 \mathrm{E}-02$} \\
\hline Basic Event, $V_{k} \quad$ Probability, $P\left(V_{k}\right)$ & RAW, $I_{R A W, k}$ \\
\hline$V_{1}$ (MDP 1 fails) & $1.07 \mathrm{E}+00$ \\
\hline $\begin{array}{l}V_{2} \text { (MDPs } 1 \text { and } 2 \text { fail) } \\
\text { (Note: Probabilities } P\left(V_{k}\right) \text { were calculated by RiskSpect }\end{array}$ & 9.64E-06 \\
\hline $\begin{array}{l}\text { Part 2: Direct Calculations by Conditional Top Event } \\
\text { Probabilities (Full RiskSpectrum }{ }^{\circledR} \text { PRA Model } \\
\text { Requantification) }\end{array}$ & $\begin{array}{l}\text { Part 3: Calculations by Formulas Using the } \\
\text { Information from PRA Model and Its Results }\end{array}$ \\
\hline \multicolumn{2}{|c|}{ Fractional Contribution, $I_{f c}$ (2. Failure or Unavailability with CCF Potential) } \\
\hline $\begin{array}{l}A=0 \text { implies } V_{k}=0 \text { for every } k, k=1, \ldots, M \text {. Set both } \\
\text { CCF basic events, } V_{1} \text { and } V_{2} \text { to "false" state and rerun: } \\
P(B \mid \bar{A})=4.0451 \mathrm{E}-05 \\
\text { Expression (B-1): } I_{f c} \approx 1-\frac{P(B \mid \bar{A})}{P(B)}=\mathbf{2 . 4 7 E - 0 5} \\
\text { (See discussion under text of Example B7.2.) }\end{array}$ & $\begin{array}{l}\text { Expression (8-8): } I_{f c}=\sum_{k=1}^{M} I_{f c, k}=1.83 \mathrm{E}-05 \\
\text { (See discussion under text of Example B7.2.) }\end{array}$ \\
\hline \multicolumn{2}{|c|}{ Risk Achievement Worth, $I_{R A W}$ (2. Failure or Unavailability with CCF Potential) } \\
\hline $\begin{array}{l}\text { Modify nominal probabilities } P\left(V_{k}\right) \text { to conditional } \\
\frac{P\left(V_{k}\right)}{P(A)} \text { for both basic events } V_{1} \text { and } V_{2} \text { and rerun: } \\
P(B \mid A)=4.6639 \mathrm{E}-05 \\
I_{R A W}=\frac{P(B \mid A)}{P(B)}=1.15 \mathrm{E}+00\end{array}$ & $\begin{array}{l}\text { Expression (8-9): } \\
I_{R A W}=1+I_{f c}\left(\frac{1}{P(A)}-1\right)=1.15 \mathrm{E}+00\end{array}$ \\
\hline \multicolumn{2}{|c|}{ Risk Reduction Worth, $I_{R R W}$ (2. Failure or Unavailability with CCF Potential) } \\
\hline$I_{R R W}=\frac{P(B)}{P(B \mid \bar{A})} \approx 1.00 \mathrm{E}+00$ & $\begin{array}{l}\text { Expression }(8-11): I_{R R W}=\frac{1}{1-I_{f c}} \approx 1.00 \mathrm{E}+00 \\
\text { Theoretical relation to } I_{R A W},(5-9): \\
I_{R R W}=\frac{1-P(A)}{1-P(A) I_{R A W}} \approx 1.00 \mathrm{E}+00\end{array}$ \\
\hline \multicolumn{2}{|c|}{ Reliability Importance, $I_{r e l}$ (2. Failure or Unavailability with CCF Potential) } \\
\hline $\begin{array}{l}\text { Multiply both basic event probabilities, } P\left(V_{1}\right) \text { and } \\
P\left(V_{2}\right) \text {, by sensitivity factor } \sigma=10 \text { and rerun: } \\
P_{U}(B)=4.0459 \mathrm{E}-05 \\
\text { Expression }(10-2): I_{r e l} \approx \frac{P_{U}(B)-P(B)}{(\sigma-1) P(A)}=6.48 \mathrm{E}-06 \\
\text { (See discussion under text of Example B7.2.) }\end{array}$ & $\begin{array}{l}\text { Expression }(8-12) \text { : } \\
I_{r e l}=P(B) \frac{I_{f c}}{P(A)}=6.16 \mathrm{E}-06\end{array}$ \\
\hline
\end{tabular}




\section{Table B-7 Example B7.3: EDG fails to start.}

\begin{tabular}{|c|c|}
\hline Part 1: Information from PRA Model and Its Results & \\
\hline CCF Model Parameters: $Q_{t}=P(A)=7.00 \mathrm{E}-03 ; \alpha_{2}=3.5$ & $\mathrm{E}-02$ \\
\hline Probability, $P\left(V_{k}\right)$ & RAW, $I_{R A W, k}$ \\
\hline$V_{1}$ (EDG 1 fails) & $3.35 \mathrm{E}-02$ \\
\hline $\begin{array}{l}V_{2} \text { (EDGs } 1 \text { and } 2 \text { fail) } \\
\text { (Note: Probabilities } P\left(V_{k}\right) \text { were calculated by RiskSpec }\end{array}$ & um ${ }^{\circledast}$ in accordance with (B-3).) \\
\hline $\begin{array}{l}\text { Part 2: Direct Calculations by Conditional Top Event } \\
\text { Probabilities (Full RiskSpectrum }{ }^{\circledR} \text { PRA Model } \\
\text { Requantification) }\end{array}$ & $\begin{array}{l}\text { Part 3: Calculations by Formulas Using the } \\
\text { Information from PRA Model and Its Results }\end{array}$ \\
\hline Fractional Contribution, $I_{f c}$ (2. Failu & or Unavailability with CCF Potential) \\
\hline $\begin{array}{l}A=0 \text { implies } V_{k}=0 \text { for every } k, k=1, \ldots, M \text {. Set both } \\
\text { CCF basic events, } V_{1} \text { and } V_{2} \text { to "false" state and rerun: } \\
P(B \mid \bar{A})=3.5999 \mathrm{E}-05 \\
\text { Expression }(10-1):{ }_{I_{f c}} \approx 1-\frac{P(B \mid \bar{A})}{P(B)}=\mathbf{1 . 1 0 E}-01\end{array}$ & Expression (8-8): $I_{f c}=\sum_{k=1}^{M} I_{f c, k}=1.11 \mathrm{E}-01$ \\
\hline Risk Achievement Worth, $I_{R A W}$ (2. Fai & re or Unavailability with CCF Potential) \\
\hline $\begin{array}{l}\text { Modify nominal probabilities } P\left(V_{k}\right) \text { to conditional } \\
\frac{P\left(V_{k}\right)}{P(A)} \text { for both basic events } V_{1} \text { and } V_{2} \text { and re-quantify } \\
\text { CDF: } \\
P(B \mid A)=6.6842 \mathrm{E}-04 \\
I_{R A W}=\frac{P(B \mid A)}{P(B)}=1.65 \mathrm{E}+01\end{array}$ & $\begin{array}{l}\text { Expression (8-9): } \\
I_{R A W}=1+I_{f c}\left(\frac{1}{P(A)}-1\right)=1.67 \mathrm{E}+01\end{array}$ \\
\hline Risk Reduction Worth, $I_{R R W}$ (2. Failu & or Unavailability with CCF Potential) \\
\hline$I_{R R W}=\frac{P(B)}{P(B \mid \bar{A})} \approx 1.12 \mathrm{E}+00$ & $\begin{array}{l}\text { Expression (8-11): } I_{R R W}=\frac{1}{1-I_{f c}} \approx 1.12 \mathrm{E}+00 \\
\text { Theoretical relation to } I_{R A W},(8-9): \\
I_{R R W}=\frac{1-P(A)}{1-P(A) I_{R A W}} \approx 1.12 \mathrm{E}+00\end{array}$ \\
\hline Reliability Importance, $I_{r e l}$ (2. Failu & or Unavailability with CCF Potential) \\
\hline $\begin{array}{l}\text { Multiply both basic event probabilities, } P\left(V_{1}\right) \text { and } \\
P\left(V_{2}\right) \text {, by sensitivity factor } \sigma=10 \text { and rerun: } \\
P_{U}(B)=8.0443 \mathrm{E}-05 \\
\text { Expression }(\mathrm{B}-2): I_{r e l} \approx \frac{P_{U}(B)-P(B)}{(\sigma-1) P(A)}=6.35 \mathrm{E}-04\end{array}$ & $\begin{array}{l}\text { Expression (8-12): } \\
I_{r e l}=P(B) \frac{I_{f c}}{P(A)}=6.39 \mathrm{E}-04\end{array}$ \\
\hline
\end{tabular}




\section{Risk Importance Measures in the Design and Operation of NPPs}

Table B-8 Example B7.4: EDG fails to run for a mission time.

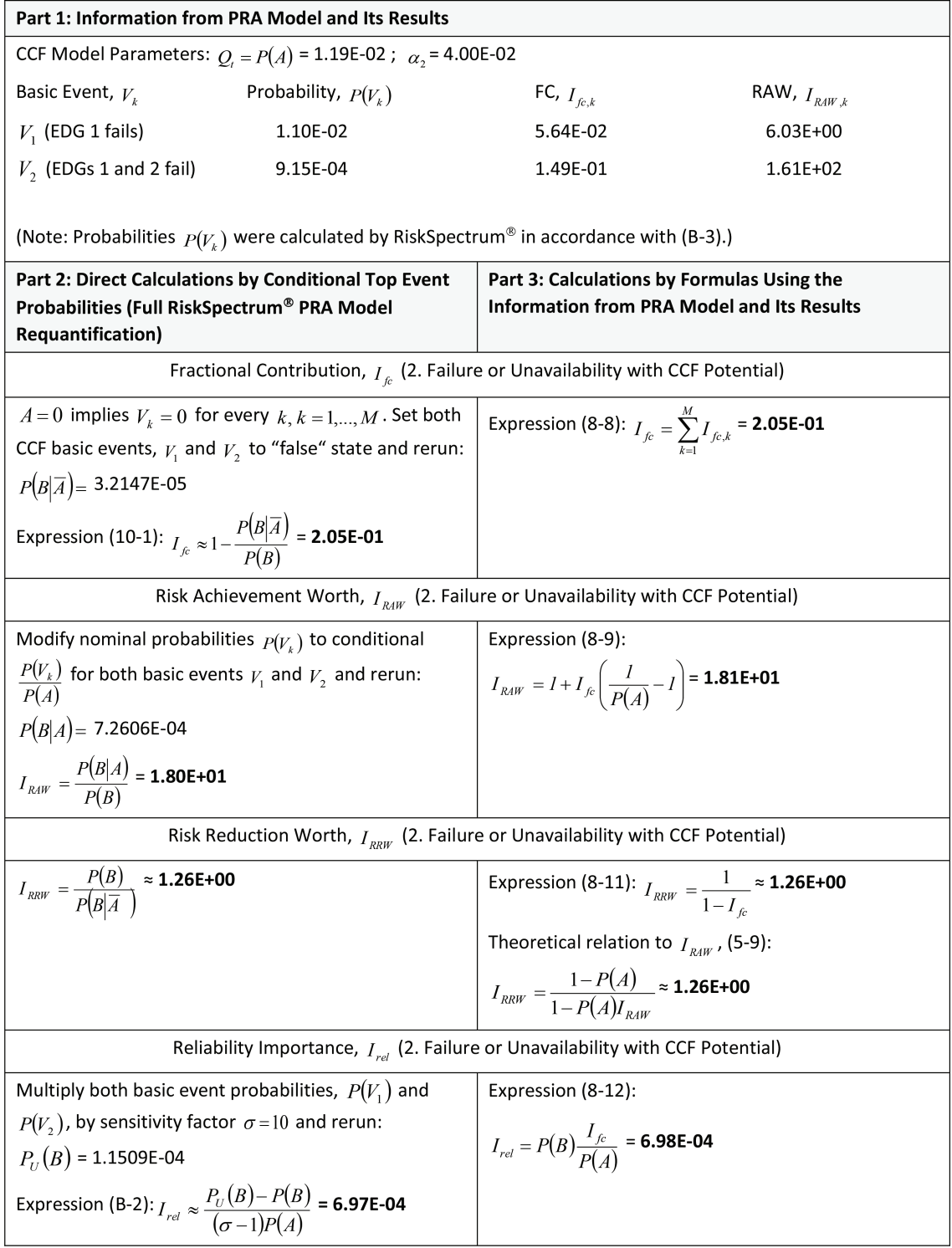




\section{Table B-9 Example B7.5: EFW discharge check valve fails to open.}

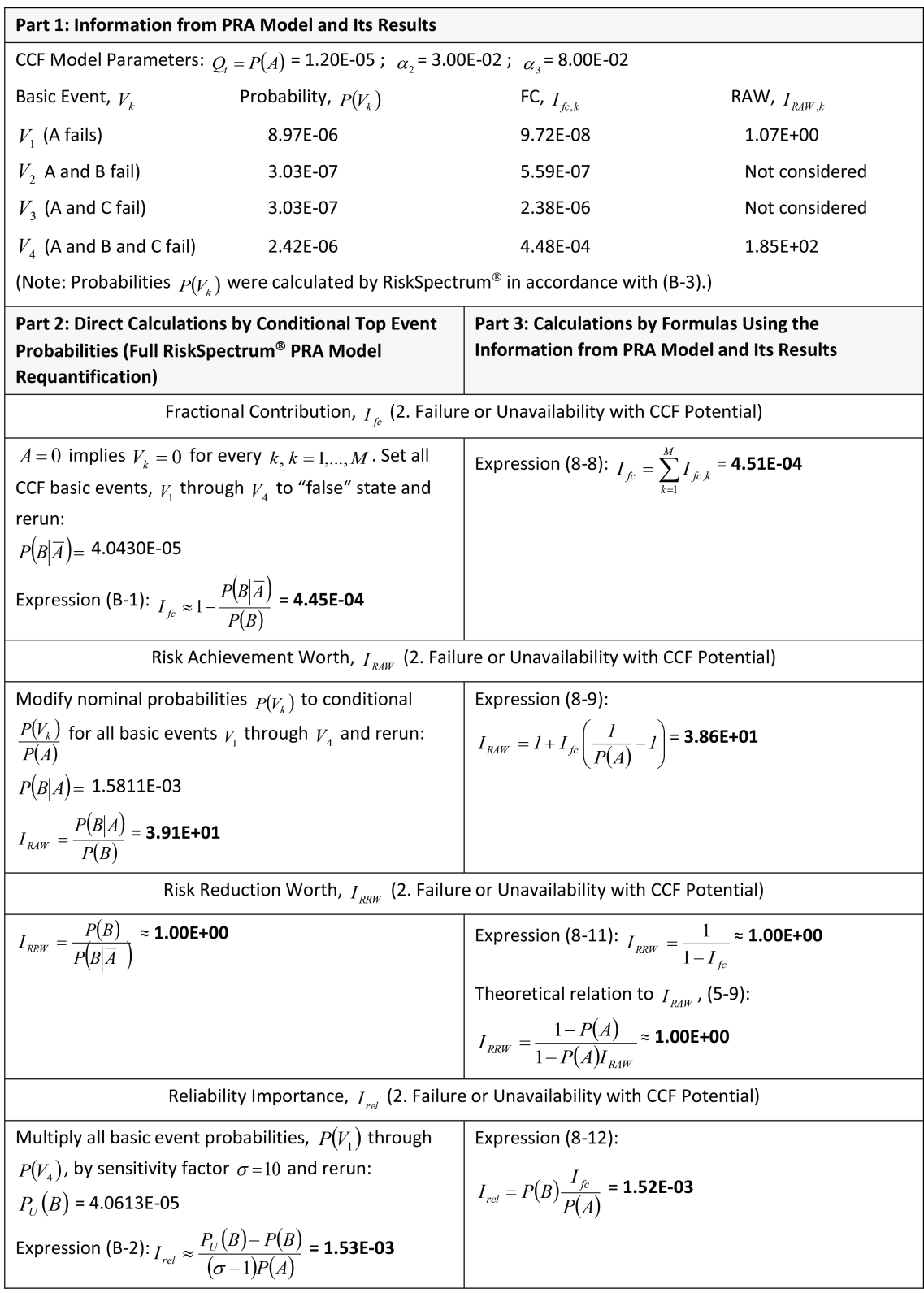


calculated by the mentioned formulas against the importance measures calculated on the basis of full PRA model re-quantification.

Each example is presented in the form of a table consisting of three main parts.

Part 1 presents the necessary information taken from the referential PRA model (described in Section B.1) which, typically, consists of probabilities and importance measures of basic events related to a considered component. It is noted that $I_{f_{c}}$ corresponds to the Fussel-Vesely importance measure reported by RiskSpectrum ${ }^{\circledR}$, as already explained under Example B1.

Part 2 describes calculation of the four importance measures based on the full re-quantification of the PRA model. These calculations rely on the top event ("reactor core damage") conditional probabilities which are obtained from the appropriately modified PRA model. (Mind the discussion on top event frequency vs. top event probability in 5.1.) Basically, RAW and RRW are calculated directly from the theoretical definitions (5-4) and (5-5), i.e. $I_{R A W}=\frac{P(B \mid A)}{P(B)}$ and $I_{R R W}=\frac{P(B)}{P(B \mid \bar{A})}$ where:

$P(B)$ : Nominal CDF of 4.0448E-05 (/yr), obtained at cutoff of 1E-12. (Refer to Section B.1.) In some instances the calculations were done at cutoff of $1 \mathrm{E}-20(\mathrm{CDF}=4.0452 \mathrm{E}-05(/ \mathrm{yr})$, Section B.1.) These are specifically pointed out.

$P(B \mid A)$ : Conditional CDF given component is failed. This was obtained by re-running the PRA model in which the component had been "failed" by setting the appropriate failure basic event(s) to the logic state "true" or by appropriately modifying their probabilities (in the case of a CCF).

$P(B \mid \bar{A})$ : Conditional CDF given component never fails. This was obtained by re-running the PRA model in which the component's failure had been precluded by setting the appropriate failure basic event(s) to the logic state "false".

FC is calculated, considering (5-16), as

$$
I_{f_{c}}=\frac{I_{F C}}{P(B)} \approx \frac{P(B)-P(B \mid \bar{A})}{P(B)}=1-\frac{P(B \mid \bar{A})}{P(B)}
$$


(where $P(B \mid \bar{A})$, obtained as described above, is taken in place of the term $P(L))$.

Reliability importance is calculated as:

$$
I_{\text {rel }} \approx \frac{\Delta P(B)}{\Delta P(A)}=\frac{P_{U}(B)-P(B)}{(\sigma-1) P(A)}
$$

where $P(A)$ refers to the overall failure probability of considered component, $\sigma$ represents certain sensitivity factor $(\sigma>1)$ and $P_{U}(B)$ is $\mathrm{CDF}$ recalculated with overall failure probability for considered component raised to $\sigma P(A)$.

Finally, in the Part 3 the importance measures are calculated from the basic information presented in the first part, by means of corresponding formulas from Sections 6 through 9 .

Any differences which can be observed between the results (importance measures) in Part 2 and those in Part 3 should be viewed in the light of errors brought in from sources such as rounding, MCS cutoff, MCS modularization process in RiskSpectrum ${ }^{\circledR}$ and MCUB approximation itself. These differences are considered to be such that they should not make any relevant impact in practical applications.

Example B5: Component-level importance measures for CVCS positive displacement pump (FT module with "OR" gate, not involved in CCF Group)

This example demonstrates calculation of importance measures for the CVCS PDP which is in the referential PRA model represented by the FT module shown in Figure 6-1. It is noted that for the FT module expressed by (6-7), $A^{*}=0$ implies $A_{i}=0$ for every $i, i=1, \ldots, n$. This is used to directly calculate the FC by re-quantifying the referential PRA model. On the other hand, for the same FT module, $A^{*}=1$ implies $A_{i}=1$ for at least one $i, i=1, \ldots, n$. This is used to directly calculate the RAW by re-quantifying the referential PRA model.

Input information, calculations and results are described in Table B-3. As it can be seen, the results obtained by formulas from Section 6 (Part 3) very closely follow those from the "full PRA model requantification" (Part 2). The differences are attributable to the errors and approximations mentioned above. However, the actual point to be made is: 
+ Calculations under Part 2 require modifications to be done to the PRA model, followed by its full requantification (or, at least, MCS post-processing).

- On the other hand, calculations under Part 3 are done by means of formulas, developed in this monograph, into which input is the information readily available: probabilities of basic events and their importance measures reported by a PRA tool. This is a significantly simpler way to calculate the measures, which can be implemented in an automated manner within the PRA tool.

Example B6: Component-level importance measures for the case of CVCS PDP with different operating failure rates (different FT Modules applied in different event sequences, not involved in CCF Group) This example shows calculation of importance measures for the CVCS PDP in the case described in Section 7.5. Since the operating failure rate for the $\mathrm{PDP}$ was modified in the event sequences originating from the total loss of ESW or CCW the CDF had to be recalculated. Thus, for this case, it was obtained:

$$
P(B)=4.3641 \mathrm{E}-05
$$

It is noted that the statement " $A$ * is true" implies that all terms (different FT modules) $A_{i}^{*}, i=1, \ldots, n$ are "true" (because the modules are "challenged" in a mutually exclusive manner). As both modules are "OR" gates, this condition can be simulated in the PRA model by setting at least one basic event from each module to "true" state. This was used for direct calculation of component-level RAW by re-quantifying the PRA model.

On the other hand, the statement " $A$ " is false" implies that all terms (different FT modules) $A_{i}^{*}, i=1, \ldots, n$ are "false". As both modules are OR-gates, this condition can be simulated in the PRA model by setting all the basic events from each module to "false" state. This was used for direct calculation of component-level RRW.

Input information, calculations and results are described in Table B-4. As can be seen from comparison of Part 2 to Part 3, the corresponding calculated values are the same or very close to each other. Table, also, gives an idea of how simple it is to use the formulas (Part 3 ) as compared to modifying and rerunning the PRA model (Part 2). 
Example B7: Importance measures for a component represented

by a single basic event (failure mode) involved in a CCF Group

In this example we demonstrate use of the formulas developed in Section 8 for importance measures for a component represented by a single basic event involved in a CCF group. Provided below are several examples and in each example considered are three types of unavailability discussed in Section 8:

1. Unavailability with no impact on CCF (preventive maintenance).

2. Failure or unavailability with CCF potential.

3. Particular CCF event: unavailability of all components included in the CCF group.

(Note that the order of the types has been changed, as compared to Section 8.)

For the reasons discussed in Sections 8.3 and 8.4, all importance measures are calculated only for the second type of unavailability. For the first and the third type provided is only RAW, which is obtained straightforwardly from the basic events importance report and is used to put into perspective the RAW for the second type, i.e. unavailability with CCF potential. The first type results with smallest and the third type with largest RAW. The RAW for the second type can be considered as some sort of "geometric mean" of the other two.

As in other examples, the risk measures calculated by the formulas are compared against the values which were obtained by full requantification of the referential PRA model (Section B.1), developed on and run by the RiskSpectrum ${ }^{\circledR}$ tool. Just as a reminder: As discussed in the introduction to Section B.3, "full requantification of PRA model" in this context involves appropriately modifying the model structure and then regenerating the minimal cutsets to calculate conditional top event (reactor core damage) probability.

Here, two points need to be emphasized.

- The first point is related to the fact that RiskSpectrum ${ }^{\circledR}$, for particular basic event " $A$ " involved in a CCF group, automatically creates and incorporates in the fault tree structure the set of CCF events $V_{k}=U_{k} A, k=1, \ldots, M$ from (8-2). Basic event " $A$ " which is directly shown in FT structure is only a front for the set of CCF 
events, which are handled automatically. When this front event " $A$ " is set to "true" state and top event (reactor core damage) requantified, RiskSpectrum ${ }^{\circledR}$ retains the probabilities of all CCF events shared by other components (for convenience here labeled as $V_{k}$ where $\left.k=2, \ldots, M\right)$ as they are, and regenerates MCSs. The final effect is as if the redundancy has been reduced by one level (e.g. from 2 to 1 ). In other wordsः status "true" of considered component is propagated under the assumption that component has been taken out for preventive maintenance. This being so, it was not possible to quantify the conditional top event probability for the case of unavailability with CCF potential (second of the three types above) in any way other than to explicitly model the basic events $V_{k}=U_{k} A, k=1, \ldots, M$ in the fault trees. The condition was then simulated by modifying their probabilities from the nominal $P\left(V_{k}\right)$ to conditional $\frac{P\left(V_{k}\right)}{P(A)}\left(=P\left(U_{k}\right)\right)$.

+ The second point concerns the appearance in the same minimal cutset of the CCF events which are considered to be mutually exclusive. For example, if CCF group contains four components, A, B, C and D, the list will contain the MCSs of the type, $\operatorname{CCF}(A B) \times C C F(B C) \times D$ etc. (involving products of CCF events which include same component). The events such as $C C F(A B)$ and $C C F(A C)$ should be considered mutually exclusive (unless defined very specifically). Thus, NUREG/ CR-5845 omits those cutsets (see Table B-1/ Table 5-2, Ref. [36]) and refers to them as to cutsets of "questionable validity” (page 38, same reference). Also, as already noted, derivations of formulas within MGL and Alpha-Factor approaches assume that these kinds of events are mutually exclusive. RiskSpectrum ${ }^{\circledR}$ would normally generate such cutsets and include them in the list. (This is not an issue with RiskSpectrum ${ }^{\circledR}-$ any PRA tool would normally do the same, unless such MCSs are precluded or deleted from the list by certain rules.) The experience with use of RiskSpectrum ${ }^{\circledR}$ and with quantification of PRA models in general shows the following: while such invalid MCS can have significant impact on the calculated $F C$ values of some of $V_{k}$ events (most notably those representing CCF of two 
components), the final impact on the overall component-level importance measures (when calculated from FC values of those $V_{k}$ events) is, in most cases, not observable.

Quantification of CCF probabilities is, in the examples which follow, done by alpha-factors, where:

$$
Q_{k}^{(m)}=\frac{k}{\left(\begin{array}{c}
m-1 \\
k-1
\end{array}\right)} \frac{\alpha_{k}}{\alpha_{t}} Q_{t}, \quad k=1, \ldots, m, \quad \text { with } \alpha_{t}=\sum_{k=1}^{m} k \alpha_{k}
$$

In the above equation the terms $Q_{k}^{(m)}, k=1, \ldots, m$ represent CCFs of specific $k$ out of all $m$ components; the term $Q_{t}=P(A)$ represents the total failure probability of considered component, and the terms $\alpha_{k}, k=1, \ldots, m$ represent alpha-factors for the considered component type.

Example B7.1: EFW MDP failure to start

In the first example of this type the importance measures are calculated for a failure to start of EFW MDP 1 (designated as "3PMPA" in Figure B-2) which is involved in the CCF group with failure to start of MDP 2 (designated as "3PMPB" in the same figure). The results are shown in Table B-5. As already pointed, the importance measures were calculated for the above mentioned unavailability type 2 (with CCF potential).

The RAW for the type "no CCF potential" (preventive maintenance) is directly obtained from the basic events importance list created by the RiskSpectrum ${ }^{\circledR}$ (at model quantification) as RAW for the basic event " $V_{1}$ " ("MDP 1 fails") and the RAW for a CCF for both pumps is directly obtained from the same list as RAW for the basic event " $V_{2}$ " ("MDPs 1 and 2 fail"). Both values are provided in Part 1 of the table. The RAW values for the three unavailability types can be summarized as:

\begin{tabular}{|c|c|c|}
\hline \multicolumn{3}{|c|}{ RAW } \\
\hline $\begin{array}{c}\text { 1. Preventive } \\
\text { Maintenance }\end{array}$ & 2. CCF Potential & $\begin{array}{c}\text { 3. CCF of Both } \\
\text { Pumps }\end{array}$ \\
\hline 1.07 & $\mathbf{1 . 4 8}$ & 3.16 \\
\hline
\end{tabular}

As expected, the first type of unavailability results with smallest RAW and the third with largest. The RAW for the second type (which 
is, actually, the real representative of RAW for this failure mode) is some sort of "geometric mean" of the other two.

Regarding the other importance measures, it is pointed out that care is needed when reliability importance $\left(I_{\text {rel }}\right)$ is used and interpreted in a case with CCF involvement. Namely, the definition of $I_{\text {rel }}$ implies that total failure probability $Q_{t}=P(A)$ is varied only for the considered component while it remains the same for other CCF members. This is contrary to the practice of symmetric CCF modeling (i.e. all members of a CCF group are assigned the same $Q_{t}$ ) which is many times used in the PRA. Possible implications may need to be considered.

Example B7.2: EFW MDP failure to run for mission time

Next example shows calculation of the importance measures for a failure of the same EFW MDP 1 to run for a mission time which is also involved in the CCF group with failure to run of MDP 2 (Table B-6). Analogous considerations apply as in previous example.

It should be noted that RAW values for preventive maintenance and CCF of both pumps are the same as in Example B7.1, for the reasons discussed in Sections 9.3 and 9.4. The RAW for the case with CCF potential is different due to different alpha-factors for the two failure modes. The RAWs for the three unavailability tapes are summarized as:

\begin{tabular}{|c|c|c|}
\hline \multicolumn{3}{|c|}{ RAW } \\
\hline $\begin{array}{c}\text { 1. Preventive } \\
\text { Maintenance }\end{array}$ & 2. CCF Potential & $\begin{array}{c}\text { 3. CCF of Both } \\
\text { Pumps }\end{array}$ \\
\hline 1.07 & $\mathbf{1 . 1 5}$ & 3.16 \\
\hline
\end{tabular}

The same observations concerning the RAWs apply as in previous example.

Unfortunately, the risk decrease importance for this failure mode is very low (FC values are of the order of 1E-05) which does not make it very good example for demonstration purposes. This reflects in the fact that difference $P(B)-P(B \mid \bar{A})$ (needed for verification of FC) cannot be reliably calculated as it is comparable to the rounding errors and errors brought in by the MCUB approximation. It is similar with the difference $P_{U}(B)-P(B)$ needed for the verification of reliability importance. This is so even with cutoff which was, for this example, lowered 
to $1 \mathrm{E}-20$, giving $P(B)=4.0452 \mathrm{E}-05$ (as compared to $4.0448 \mathrm{E}-05$ at $1 \mathrm{E}-12)$.

Example B7.3: Emergency diesel generator (EDG) failure to start In the third example the importance measures are calculated for a failure to start of EDG 1 which is involved in the CCF group with failure to start of EDG 2. The results are shown in Table B-7. The RAW values for the three unavailability types can be summarized as:

\begin{tabular}{|c|c|c|}
\hline \multicolumn{3}{|c|}{ RAW } \\
\hline $\begin{array}{l}\text { 1. Preventive } \\
\text { Maintenance }\end{array}$ & 2. CCF Potential & $\begin{array}{c}\text { 3. CCF of Both } \\
\text { EDGs }\end{array}$ \\
\hline 6.03 & $\mathbf{1 6 . 7}$ & 161 \\
\hline
\end{tabular}

Once again, it can be seen that representative RAW for CCF is a sort of "geometric mean" of the RAW for preventive maintenance unavailability and RAW for the total CCF event.

Example B7.4: EDG failure to run for mission time

Next example is calculation of the importance measures for a failure of EDG 1 to run for a mission time, which is involved in the CCF group with failure to run of EDG 2. The results are shown in Table B-8. The RAW values for the three unavailability types can be summarized as:

\begin{tabular}{|c|c|c|}
\hline \multicolumn{3}{|c|}{ RAW } \\
\hline $\begin{array}{c}\text { 1. Preventive } \\
\text { Maintenance }\end{array}$ & 2. CCF Potential & $\begin{array}{c}\text { 3. CCF of Both } \\
\text { EDGs }\end{array}$ \\
\hline 6.03 & $\mathbf{1 8 . 1}$ & 161 \\
\hline
\end{tabular}

The same observations apply as in previous example. Example B7.5: EFW discharge check valve failure to open For the last example in this section, calculation of importance measures for a component involved in a CCF group with size $m=3$ was selected. This is the discharge check valve labeled as " $32 \mathrm{~A}$ " in Figure B-2 which is involved in the CCF group with check valves " $32 \mathrm{~B}$ " and " $32 \mathrm{C}$ " (same figure). Considered is failure to open on demand. The results are shown in Table B-9. Analogous considerations apply as in the above examples. 
Note that, similarly to Example B7.2, the risk decrease importance for the failure mode is very low, which, unfortunately, does not make it particularly good example. The RAW values for the three unavailability types are summarized as:

\begin{tabular}{|c|c|c|}
\hline \multicolumn{3}{|c|}{ RAW } \\
\hline $\begin{array}{l}\text { 1. Preventive } \\
\text { Maintenance }\end{array}$ & 2. CCF Potential & 3. CCF of All Valves \\
\hline 1.07 & $\mathbf{3 8 . 6}$ & 185 \\
\hline
\end{tabular}

Example B8: Importance measures for a component represented by multiple basic events (input to "OR" gate) involved in CCF Groups

This section demonstrates the use of formulas from Section 9 for calculating the component-level importance measures for a component represented by multiple basic events input into an "OR" gate which are all members of CCF groups of the size $m$. In other words, the component is involved in the CCF group of $m$ redundant components. (It is noted, however, that formulas can also be applied if some of the basic events input into an "OR" gate are not involved in CCF groups.) There are two examples, one with two redundant MDPs and the other with two redundant EDGs. Analogous considerations apply as in Example B7.

Example B8.1: One of two redundant EFW MDPs

In this example we calculate the component-level importance measures for EFW MDP 1 (designated as "3PMPA" in Figure B-2) which is involved in the CCF group with MDP 2 (designated as "3PMPB" in the same figure). It is represented by two basic events in the FTs: failure to start and failure to run for a mission time. Each is member of corresponding CCF group. Importance measures for these basic events were calculated in Examples B7.1 and B7.2. The component-level results are shown in Table B-10.

The RAW values for the three unavailability types are summarized below. It should be noted that component-level RAW values for preventive maintenance and CCF of both pumps are the same as in Examples B7.1 and B7.2, as already discussed in Sections 9.3 and 9.4. 


\section{Table B-10 Example B8.1: Component-level importance measures for EFW MDP1.}

\begin{tabular}{|c|c|}
\hline Part 1: Information from PRA Model and Its Results & \\
\hline $\begin{array}{l}\text { All necessary information was provided under Examples } \\
\text { failure probability is calculated as: } P(A) \approx P\left(A_{F S}\right)+P\left(A_{F R}\right.\end{array}$ & $\begin{array}{l}\text { B7.1 and B7.2. According to (9-2) component-level } \\
=Q_{t, F S}+Q_{t, F R}=2.00 \mathrm{E}-03+1.20 \mathrm{E}-04=2.12 \mathrm{E}-03\end{array}$ \\
\hline $\begin{array}{l}\text { Part 2: Direct Calculations by Conditional Top Event } \\
\text { Probabilities (Full RiskSpectrum }{ }^{\circledR} \text { PRA Model } \\
\text { Requantification) }\end{array}$ & $\begin{array}{l}\text { Part 3: Calculations by Formulas Using the } \\
\text { Information from PRA Model and Its Results }\end{array}$ \\
\hline Risk Achievement Worth, $I_{R A W}$ (2. Fail & re or Unavailability with CCF Potential) \\
\hline $\begin{array}{l}\text { Modify } P\left(A_{s}\right) \text { from } Q_{t, s} \text { to } H_{s} \text { for basic events related } \\
\text { to EFW MDP } 1 \text { and propagate this through the } \\
\text { corresponding } P\left(V_{k, s}\right) \text {, for both } s \equiv F S \text { and } s \equiv F R \text {. } \\
\text { Practically: replace } P\left(V_{k, s}\right) \text { with } P\left(V_{k, s}\right) \frac{H_{s}}{Q_{t, s}} \text {. } \\
\text { Requantify CDF: } \\
P(B \mid A)=5.9368 \mathrm{E}-05 \\
I_{R A W}=\frac{P(B \mid A)}{P(B)}=\mathbf{1 . 4 7 E + 0 0}\end{array}$ & $\begin{array}{l}\text { Calculate conditional probabilities for failure modes, } \\
\text { (9-7): } \\
H_{s}=\frac{P\left(A_{s}\right)}{\sum_{s=1}^{S} P\left(A_{s}\right)}, H_{F S}=9.43 \mathrm{E}-01 ; H_{F R}=5.66 \mathrm{E}-02 \\
\text { From (9-6): } \\
I_{R A W}=\sum_{s=1}^{S} H_{s} I_{R A W, s}=H_{F S} I_{R A W, F S}+H_{F R} I_{R A W, F R} \\
\text { Take } I_{R A W, F S} \text { and } I_{R A W, F R} \text { from Example B7.1 and B7.2: } \\
I_{R A W}=1.46 \mathrm{E}+00\end{array}$ \\
\hline Risk Reduction Worth, $I_{R R W}$ (2. Failur & or Unavailability with CCF Potential) \\
\hline $\begin{array}{l}A=0 \text { implies } V_{k, s}=0 \text { for every } k, k=1, \ldots, M \text { and } \\
s, s=1, \ldots, S . \text { Set all basic events, } V_{1, F S}, V_{2, F S}, V_{1, F R} \text {, and } \\
V_{2, F R} \text { to "false" state. Requantify CDF: } \\
P(B \mid \bar{A})=4.0408 \mathrm{E}-05 \\
I_{R R W}=\frac{P(B)}{P(B \mid \bar{A})} \approx 1.00 \mathrm{E}+00\end{array}$ & $\begin{array}{l}\text { Theoretical relation to } I_{R A W},(5-9) \text { : } \\
I_{R R W}=\frac{1-P(A)}{1-P(A) I_{R A W}} \approx 1.00 \mathrm{E}+00\end{array}$ \\
\hline Fractional Contribution, $I_{f c}$ (2. Failu & or Unavailability with CCF Potential) \\
\hline Expression (B-1): $I_{f_{c}} \approx 1-\frac{P(B \mid \bar{A})}{P(B)}=\mathbf{9 . 8 9 E - 0 4}$ & $\begin{array}{l}\text { Expression (9-11): } I_{f c}=\frac{I_{R A W}-1}{\frac{1}{P(A)}-1}=\mathbf{9 . 8 2 \mathrm { E } - 0 4} ; \\
\text { Perform cross-check by (9-9). Take } I_{f c, F S} \text { and } I_{f c, F R} \\
\text { from Examples B7.1 and B7.2: } \\
I_{f c}=\sum_{s=1}^{S} I_{f c, s}=I_{f c, F S}+I_{f c, F R}=\mathbf{9 . 8 2 \mathrm { E } - 0 4}\end{array}$ \\
\hline Reliability Importance, $I_{r e l}$ (2. Failure & or Unavailability with CCF Potential) \\
\hline $\begin{array}{l}\text { Multiply all basic event probabilities, } P\left(V_{1, F S}\right), P\left(V_{2, F S}\right) \text {, } \\
P\left(V_{1, F R}\right) \text {, and } P\left(V_{2, F R}\right) \text {, by sensitivity factor } \sigma=10 . \\
\text { Requantify CDF: } \\
P_{U}(B)=4.0809 \mathrm{E}-05 \\
\text { Expression }(\mathrm{B}-2): I_{r e l} \approx \frac{P_{U}(B)-P(B)}{(\sigma-1) P(A)}=\mathbf{1 . 8 9 \mathrm { E } - 0 5}\end{array}$ & $\begin{array}{l}\text { Analogously to expression (8-12): } \\
I_{r e l}=P(B) \frac{I_{f c}}{P(A)}=1.87 \mathrm{E}-05\end{array}$ \\
\hline
\end{tabular}




\section{Risk Importance Measures in the Design and Operation of NPPs}

\section{Table B-11 Example B8.2: Component-level importance measures for EDG 1 .}

\begin{tabular}{|c|c|}
\hline \multicolumn{2}{|l|}{ Part 1: Information from PRA Model and Its Results } \\
\hline \multicolumn{2}{|c|}{$\begin{array}{l}\text { All necessary information was provided under Examples B7.3 and B7.4. According to (9-2) component-level } \\
\text { failure probability is calculated as: } P(A) \approx P\left(A_{F S}\right)+P\left(A_{F R}\right)=Q_{t, F S}+Q_{t, F R}=7.00 \mathrm{E}-03+1.19 \mathrm{E}-02=1.89 \mathrm{E}-02\end{array}$} \\
\hline $\begin{array}{l}\text { Part 2: Direct Calculations by Conditional Top Event } \\
\text { Probabilities (Full RiskSpectrum }{ }^{\circledR} \text { PRA Model } \\
\text { Requantification) }\end{array}$ & $\begin{array}{l}\text { Part 3: Calculations by Formulas Using the } \\
\text { Information from PRA Model and Its Results }\end{array}$ \\
\hline \multicolumn{2}{|c|}{ Risk Achievement Worth, $I_{R A W}$ (2. Failure or Unavailability with CCF Potential) } \\
\hline $\begin{array}{l}\text { Modify } P\left(A_{s}\right) \text { from } Q_{t, s} \text { to } H_{s} \text { for basic events related } \\
\text { to EDG } 1 \text { and propagate this through the } \\
\text { corresponding } P\left(V_{k, s}\right) \text {, for both } s \equiv F S \text { and } s \equiv F R \text {. } \\
\text { Practically: replace } P\left(V_{k, s}\right) \text { with } P\left(V_{k, s}\right) \frac{H_{s}}{Q_{t, s}} \text {. } \\
\text { Requantify CDF: } \\
P(B \mid A)=7.0478 \mathrm{E}-04 \\
I_{R A W}=\frac{P(B \mid A)}{P(B)}=\mathbf{1 . 7 4 E + 0 1}\end{array}$ & $\begin{array}{l}\text { Calculate conditional probabilities for failure modes, } \\
\text { (9-7): } \\
H_{s}=\frac{P\left(A_{s}\right)}{\sum_{s=1}^{S} P\left(A_{s}\right)}, H_{F S}=3.70 \mathrm{E}-01 ; H_{F R}=6.30 \mathrm{E}-01 \\
\text { From (9-6): } \\
I_{R A W}=\sum_{s=1}^{S} H_{s} I_{R A W, s}=H_{F S} I_{R A W, F S}+H_{F R} I_{R A W, F R} \\
\text { Take } I_{R A W, F S} \text { and } I_{R A W, F R} \text { from Example B7.3 and B7.4: } \\
I_{R A W}=1.75 \mathrm{E}+01\end{array}$ \\
\hline \multicolumn{2}{|c|}{ Risk Reduction Worth, $I_{R R W}$ (2. Failure or Unavailability with CCF Potential) } \\
\hline $\begin{array}{l}A=0 \text { implies } V_{k, s}=0 \text { for every } k, k=1, \ldots, M \text { and } \\
s, s=1, \ldots, S . \text { Set all basic events, } V_{1, F S}, V_{2, F S}, V_{1, F R} \text {, and } \\
V_{2, F R} \text { to "false" state. Requantify CDF: } \\
P(B \mid \bar{A})=2.7683 \mathrm{E}-05 \\
I_{R R W}=\frac{P(B)}{P(B \mid \bar{A})} \approx 1.46 \mathrm{E}+00\end{array}$ & $\begin{array}{l}\text { Theoretical relation to } I_{R A W},(5-9) \text { : } \\
I_{R R W}=\frac{1-P(A)}{1-P(A) I_{R A W}} \approx 1.47 \mathrm{E}+00\end{array}$ \\
\hline \multicolumn{2}{|c|}{ Fractional Contribution, $I_{f c}$ (2. Failure or Unavailability with CCF Potential) } \\
\hline Expression (B-1): $I_{f c} \approx 1-\frac{P(B \mid \bar{A})}{P(B)}=\mathbf{3 . 1 6 \mathrm { E } - 0 1}$ & $\begin{array}{l}\text { Expression (9-11): } I_{f c}=\frac{I_{R A W}-1}{\frac{1}{P(A)}-1}=\mathbf{3 . 1 9 \mathrm { E } - 0 1} \text {; } \\
\text { Perform cross-check by (9-9). Take } I_{f c, F S} \text { and } I_{f c, F R} \\
\text { from Examples B7.3 and B7.4: } \\
I_{f c}=\sum_{s=1}^{S} I_{f c, s}=I_{f c, F S}+I_{f c, F R}=\mathbf{3 . 1 6 \mathrm { E } - 0 1}\end{array}$ \\
\hline \multicolumn{2}{|c|}{ Reliability Importance, $I_{r e l}$ (2. Failure or Unavailability with CCF Potential) } \\
\hline $\begin{array}{l}\text { Multiply all basic event probabilities, } P\left(V_{1, F S}\right), P\left(V_{2, F S}\right) \text {, } \\
P\left(V_{1, F R}\right) \text {, and } P\left(V_{2, F R}\right) \text {, by sensitivity factor } \sigma=10 . \\
\text { Requantify CDF: } \\
P_{U}(B)=1.5361 \mathrm{E}-04 \\
\text { Expression }(\mathrm{B}-2): I_{r e l} \approx \frac{P_{U}(B)-P(B)}{(\sigma-1) P(A)}=6.65 \mathrm{E}-04\end{array}$ & $\begin{array}{l}\text { Analogously to expression (8-12): } \\
I_{r e l}=P(B) \frac{I_{f c}}{P(A)}=6.82 \mathrm{E}-04\end{array}$ \\
\hline
\end{tabular}


Appendix B 135

\begin{tabular}{|c|c|c|}
\hline \multicolumn{3}{|c|}{ RAW } \\
\hline $\begin{array}{c}\text { Preventive } \\
\text { Maintenance }\end{array}$ & CCF Potential & $\begin{array}{c}\text { CCF of Both } \\
\text { Pumps }\end{array}$ \\
\hline 1.07 & $\mathbf{1 . 4 6}$ & 3.16 \\
\hline
\end{tabular}

Example B8.2: One of two redundant EDGs

Last example shows calculation of the component-level importance measures for EDG 1 which is involved in the CCF group with EDG 2. Like in the previous example, the component is represented by two basic events in the FTs: failure to start and failure to run for a mission time. Each is member of corresponding CCF group. Importance measures for these basic events were calculated in Examples B7.3 and B7.4. The component-level results are shown in Table B-11.

The RAW values for the three unavailability types are summarized below. Analogously to the previous example, component-level RAW values for preventive maintenance and CCF of both EDGs are the same as in Examples B7.3 and B7.4.

\begin{tabular}{|c|c|c|}
\hline \multicolumn{2}{|c|}{ RAW } \\
\hline Preventive Maintenance & CCF Potential & $\begin{array}{c}\text { CCF of Both } \\
\text { EDGs }\end{array}$ \\
\hline 6.03 & $\mathbf{1 7 . 5}$ & 161 \\
\hline
\end{tabular}




\section{About the authors}

Mr. Ivan Vrbanic of APOSS, Croatia, has thirty years of experience in conducting probabilistic risk assessments (PRAs) for internal and external initiating events and hazards in nuclear power plants (NPPs). He has been a leader and participant in many projects on risk and safety analyses, their applications and reviews to enhance nuclear safety in many countries. Dr. Vrbanic is a scientific collaborator in the field of electrical engineering to the University of Zagreb, Croatia, and is a consultant to the International Atomic Energy Agency (IAEA). He received his $\mathrm{Ph} . \mathrm{D}$. in Technical Sciences in the field of Electrical Engineering from University of Zagreb, Croatia.

Mr. Pranab K. Samanta is a scientist at Brookhaven National Laboratory in New York, USA. His research interest includes probabilistic risk assessment (PRA), risk-informed regulations, and operational safety of nuclear power plants (NPPs). He has led many projects for US Nuclear Regulatory Commission on methods, tools, and reviews, and is involved in development of regulations, guidance documents, and standards relating to PRAs and their applications. Dr. Samanta supported regulatory authorities in many countries in improving operational safety in NPPs and is a consultant to the IAEA. He received his $\mathrm{Ph}$. D. in Nuclear Science and Engineering from University of Michigan, Ann Arbor, USA.

Mr. Ivica Basic of APOSS, Croatia, has an experience spanning three decades in nuclear safety analyses, evaluations and verifications. His specialization includes deterministic analyses and simulations in support of design-basis evaluations and demonstrations as well as assessments of risk from severe accidents in NPPs. He has been a leader and participant in many projects aimed at enhancing nuclear safety in a number of countries. He was a member of the international effort in development of plant-specific symptom-oriented emergency operating procedures and severe accident management guidelines. Mr. Basic is a consultant to the IAEA and received his M. Sc, degree in Technical Sciences in the field of Electrical Engineering from University of Zagreb, Croatia. 


\section{Index}

A

D

ASME PRA Standard of 2009, Design basis (DB) function, 9-10 12

B

Binary decision diagrams (BDDs), 90

Birnbaum importance, 14, 25, 40

C

$\mathrm{CDF}$ (core damage frequency), 16

CDF Contribution, 22, 31

CDF/LERF, 88-89; see also LERF

10 CFR 50.69, 15-16

Chemical and volume control system (CVCS), 45

Component-level importance measure, demonstrative examples of, 17-85

Component-level importance measures for a component which is not involved in any CCF group, 49-57

example, 54-57

FC, 51-52

introduction, 49-51

RAW, 52-54

RRW and reliability importance, 54

Component-level risk importance, 26

Conditional core damage probability (CCDP), 29-30

CTs (Completion times), 18 risks, 18-19

E

Emergency feedwater turbine driven pump (EFW TDP), 49

ET (event trees), 18

$\mathrm{F}$

FC (fractional contribution), 1314,36

FT (fault trees), 18, 27

"FT linking" approach, 33

"FT modules," 27

Fussell-Vesely (F-V) importance measure, 12, 13-14, 17

$\mathrm{H}$

High energy line break (HELB), 49 I

Inclusion-exclusion principle, 91

Initiating event (IE), 88

Inspection guidance, 23-24

Integrated decision-making panel (IDP), 16, 17

L

LERF, 88

LERF (large early release frequency), 16, 17, 24, 88, 89

Limiting Conditions for Operations (LCOs), 18

Logic function, 43

M

MCUB (Mincut UpperBound) approximation, 112, 125, 130 
140 Risk Importance Measures in the Design and Operation of NPPs

Minimal cut sets (MCSs), 18, PRA computer code risk impor$33-34$

tance measures by a, 101-103

Mitigating systems performance index (MSPI), 24

PRA model, examples based on a simplified, 105-135

$\mathrm{N}$

NEI 00-04, 16, 17-18, 29, $88-89$

NEI 97-04, 9

"Non-safety-related" equipment, $15-16$

"Non-safety" ("non-class") SSCs, 9

NPPs (nuclear power plants) overview, 1-2 safety analysis, 3, 5 technical specifications, 14 NPPs (nuclear power plants), risk assessment measures, 11-30 background, 11-12 risk-informed applications, 14 25

used in PRAs for NPPs, 12-14, 26-30

NRC Inspection Manual, 23

NRC's Maintenance Rule, 21

NRC's ROP, 22-23

NUMARC 93-01, 21-22

NUREG-1649, 22-23

NUREG/CR-3385, 13, 14

NUREG/CR-4780, 60

NUREG/CR-5497, 73

NUREG/CR-5750, 32, 107

NUREG/CR-5845, 60, 73

NUREG/CR-6928, 35-36, 107

$\mathbf{P}$

Positive displacement pump (PDP), 45

R

RAW (risk achievement worth), 13, 18, 20, 22, 28; see also Risk importance measures and RRW, 34-35

Reactor oversight process (ROP), 15,24

Risk importance measures

limitations of 87-92

by a PRA computer code, 101103

Risk importance of a component represented by multiple basic events, FT module, 43-47

example, 45-47

FC, RAW, RRW and reliability importance for component-level FT module, 44 introduction, 43-44

Risk importance of component represented by a single basic event (failure mode) involved in a CCF Group, 59-68

failure with CCF potential, 6065

introduction, 59

particular CCf event, 67-68

unavailability with no impact on CCF, 65-67

Risk importance of component represented by a single basic event not involved in a CCF Group, 31-41 
FC for a single basic event, 35-37 S

introduction, 31-34

RAW and RRW, 34-35

RAW for a single basic event, 37-38

realiability importance for a single basic event, 39-41

RRW for a single basic event, 39

Risk importance of component represented by multiple basic events (failure modes) involved in CCF groups, 69-77

failure with CCF potential, 73-76

introduction, 69-73

particular CCF event, 77

unavailability with no impact no CCF, 77

RiskSpectrum $^{\circledast}$, 12, 79, 101-103, 105, 107-108

RRW (risk reduction worth), 13, $16,17,20,22$, see also RAW (risk achievement worth); Risk importance measures
SF (Surveillance frequency), 18 risks, 20-21

SRP Section 3.2.2, 10

SSC (system, structure, and component), deterministic principles of safety importance, 7-10; see also Risk importance measures

Station blackout (SBO), 49

Surveillance requirements (SRs), 18

$\mathrm{T}$

Technical specifications (TSs) for NPPs, 18; see also NPPs (nuclear power plants)

$\mathrm{U}$

USNRC (United States Nuclear Regulatory Commission), 8 Regulatory Guide 1.26, 8 Regulatory Guide 1.70, 7

Regulatory Guide 1.160, 21-22

Regulatory Guide 1.174, 89

Standard review Plan (SRP), 8-9 